\title{
Dissipation and the Fundamental Plane: Observational Tests
}

\section{Citation}

Hopkins, Philip F., Thomas J. Cox, and Lars Hernquist. 2008. "Dissipation and the Fundamental Plane: Observational Tests." The Astrophysical Journal 689 (1): 17-48. https:// doi.org/10.1086/592105.

\section{Permanent link}

http://nrs.harvard.edu/urn-3:HUL.InstRepos:41381620

\section{Terms of Use}

This article was downloaded from Harvard University's DASH repository, and is made available under the terms and conditions applicable to Other Posted Material, as set forth at http:// nrs.harvard.edu/urn-3:HUL.InstRepos:dash.current.terms-of-use\#LAA

\section{Share Your Story}

The Harvard community has made this article openly available.

Please share how this access benefits you. Submit a story.

Accessibility 


\title{
DISSIPATION AND THE FUNDAMENTAL PLANE: OBSERVATIONAL TESTS
}

\author{
Philip F. Hopkins, ${ }^{1}$ Thomas J. Cox, ${ }^{1,2}$ and Lars Hernquist ${ }^{1}$ \\ Received 2008 June 6; accepted 2008 July 10
}

\begin{abstract}
We develop observational tests of the idea that dissipation in gas-rich mergers produces the fundamental plane (FP) and related correlations obeyed by ellipticals. The FP "tilt" implies that lower mass ellipticals have a higher ratio of stellar to dark matter within their stellar effective radii. Models argue that mergers between more gas-rich (typically lower mass) disks yield larger mass fractions formed in compact starbursts, giving a smaller stellar $R_{e}$ and higher $M_{*} / M_{\mathrm{tot}}$ within that $R_{e}$. Such starbursts leave a characteristic imprint in the surface brightness profile: a central excess above an outer profile established by the dissipationless violent relaxation of disk stars. In previous work, we developed empirical methods to decompose the observed profiles of ellipticals and robustly estimate the amount of dissipation in the original spheroid-forming merger(s). Applying this to a large sample of observed ellipticals, we test whether or not their location on the FP and its tilt are driven by dissipation. At fixed mass, ellipticals formed in more dissipational events are smaller and have higher $M_{*} / M_{\text {tot }}$. At fixed degree of dissipation, there is no tilt in the FP. We show that the dynamical mass estimator $R_{e} \sigma^{2} / G$ is a good estimator of the true mass: the observed FP tilt cannot primarily owe to other forms of nonhomology. Removing the effects of dissipation, observed ellipticals obey the same FP correlations as disks: unusual progenitors are not required to make typical ellipticals. Dissipation appears to be both necessary and sufficient to explain the FP tilt.
\end{abstract}

Subject headings: cosmology: theory — galaxies: evolution — galaxies: nuclei — quasars: general

Online material: color figures

\section{INTRODUCTION}

Understanding the scaling relations between the photometric and kinematic properties of galaxy spheroids (their masses, sizes, velocity dispersions, and luminosities) is fundamental to explaining the origin of early-type galaxies. Faber \& Jackson (1976) demonstrated that ellipticals obey a relatively tight correlation between optical luminosity and central velocity dispersion, and Kormendy (1977) found an analogous relationship between their effective surface brightness and radii. With improved observations and the advent of stellar population modeling, these observed trends can be translated into robust correlations between physical parameters: a velocity dispersion-stellar mass $\left(\sigma-M_{*}\right)$ and a sizestellar mass $\left(R_{e}-M_{*}\right)$ relation.

Djorgovski \& Davis (1987) and Dressler et al. (1987) demonstrated that the scatter in either the Faber \& Jackson (1976) or Kormendy (1977) relation could be reduced by adopting a threeparameter correlation of the form $\log R_{e}=a \log \sigma-0.4 b \mu_{e}+c$ (equivalently $R_{e} \propto \sigma^{a} I_{e}^{b}$ ), with best-fit scalings $a \sim 1.3-1.4$, $b \sim-0.8$ to -0.9 . This defines the "fundamental plane" (FP) of elliptical galaxies: a correlation relating stellar mass or luminosity (implicit in the surface brightness), effective radius, and velocity dispersion (effectively the dynamical mass of the system). With a small observed scatter $\sim 0.1$ dex, the FP has presented a long-standing and still unresolved challenge to observations and theoretical models of spheroid formation.

In developing a physical understanding of the FP and associated elliptical scaling laws, Djorgovski et al. (1988), Jorgensen et al. (1993), and others demonstrated that the FP could be represented as a "tilted" virial plane. If ellipticals were perfectly homologous systems with constant stellar mass-to-light ratios $M_{*} / L$, then a virial correlation $L \propto M_{*}=k \sigma^{2} R_{e} / G \equiv M_{\text {dyn }}$, with

\footnotetext{
1 Harvard-Smithsonian Center for Astrophysics, Cambridge, MA 02138.

2 W. M. Keck Postdoctoral Fellow at the Harvard-Smithsonian Center for Astrophysics.
}

constant integral factor $k$, would be expected. Since $I_{e} \propto L / R_{e}^{2}$, this translates to an expected "virial FP" $R_{e} \propto \sigma^{2} I_{e}^{-1}$. The observed FP is similar to this, but not exactly so; it is equivalent to and can be represented as a tilted version of this correlation, namely,

$$
M_{\text {dyn }} \propto M_{*}^{1+\alpha}
$$

with some small but nonzero $\alpha$. Equivalently, the difference between the best-fit observed FP (in any projection) and the virial FP can be expressed as a mass-dependent mass-to-light (or, for our purposes, total mass-to-stellar mass) ratio

$$
\frac{M_{\mathrm{dyn}}}{M_{*}} \propto M_{*}^{\alpha},
$$

where the quantity $\alpha$ quantifies the tilt, or deviation of the FP from the virial relation. Various independent measurements find similar values of $\alpha \approx 0.2$ (e.g., Pahre et al. 1998; Gerhard et al. 2001; Borriello et al. 2003; Padmanabhan et al. 2004; Gallazzi et al. 2006). Although this is not strictly identical to the best-fit relation $R_{e} \propto \sigma^{a} I_{e}^{b}$, if both $a$ and $b$ are fitted as free parameters, multiple observations have shown that it is statistically an equivalent representation (i.e., has the same scatter in physical quantities) and that there is no additional information in the best-fit FP beyond this tilt (i.e., once this tilt is accounted for, there is no additional systematic scaling in $R_{e}$ or $\sigma$ that can reduce the scatter in predicting the other quantities).

It is now well established that part of the observed tilt in optical bands is a consequence of stellar population effects: lower mass ellipticals tend to be younger, yielding lower stellar massto-light ratios (see, e.g., Trager et al. 2000). However, various constraints imply that only a small fraction of the optical tilt owes to these effects (see, e.g., Pahre et al. 1998; Gerhard et al. 2001; Bertin et al. 2002; Borriello et al. 2003; Padmanabhan et al. 2004; Trujillo et al. 2004; Gallazzi et al. 2006; von der Linden 
et al. 2007). For example, in the $K$ band, the tilt is still substantial: observations indicate $M_{\text {dyn }} \propto L_{K}^{1.25 \pm 0.05}$ (Pahre et al. 1998), whereas the systematic dependence of $M_{*} / L_{K}$ is quite weak (most estimates suggest $M_{*} / L_{K} \propto L_{K}^{0.03}$; Bell et al. 2003). It is now possible to combine high-resolution spectra and stellar population synthesis models, allowing reliable stellar mass estimates, and almost all such studies yield a similar relation:

$$
M_{\text {dyn }} \propto M_{*}^{1.2}
$$

i.e., $\alpha \approx 0.2$ as described above. It has been demonstrated that this result is robust to the details of the stellar population model, spectral coverage, or even simplifying assumptions such as the use of a single color to derive a mean $M_{*} / L$.

There are only two reasonable explanations for this finding (some combination of the two is also possible). First, the true mass enclosed within the stellar effective radius $\left(M_{\text {true }}\right)$ could in fact be proportional to the stellar mass $M_{*}$, but owing to, e.g., changes in the profile shape or kinematics of galaxies with mass (traditional nonhomology), the relation between actual mass and the dynamical mass estimator $M_{\mathrm{dyn}} \propto \sigma^{2} R_{e} / G$ is a changing function of mass. In other words, $M_{\text {dyn }} \propto M_{\text {true }}^{1.2}$. However, observations appear to rule out this possibility, at least as the origin of most of the tilt. Integral modeling of the mass distribution from two-dimensional kinematic maps (which should recover any systematic difference between $M_{\text {dyn }}$ and $M_{\text {true }}$ without reference to any homology assumptions; e.g., Cappellari et al. 2006), as well as mass distributions estimated from gravitational lensing (Bolton et al. 2007, 2008; Nipoti et al. 2008), independently gives $M_{\text {dyn }} \propto M_{\text {true }}^{1.00 \pm 0.03}$. That is, the allowed contribution of nonhomology to the FP tilt is small.

The only remaining explanation is that the FP tilt reflects a meaningful physical change, namely, that the ratio of total enclosed mass within $R_{e}\left(M_{\text {true }}\right.$, represented reasonably well to within a normalization constant by $\left.M_{\text {dyn }}\right)$ to the stellar mass is an increasing function of mass $\left(M_{\text {true }} \propto M_{*}^{1.2}\right)$. In other words, low-mass ellipticals are more baryon dominated within their stellar $R_{e}$, and highmass ellipticals have higher dark matter fractions. We emphasize that these constraints apply within the stellar effective radii. The change in dark matter fraction is not required to be global: if one were to contract the stellar mass distribution but keep the dark matter halo relatively fixed, for example, it would significantly decrease the dark matter fraction (and correspondingly $M_{\mathrm{dyn}} / M_{*}$ ) within $R_{e}$.

This trend is contrary to that followed by disks. For disk galaxies, an opposite (negative) tilt $M_{\text {dyn }} \propto M_{*}^{0.7}-M_{*}^{0.8}(\alpha \approx-0.2$ to -0.3 ) is observed (see, e.g., Persic et al. $1996 \mathrm{~b}$; Bell \& de Jong 2001; Shen et al. 2003; Courteau et al. 2007): low-mass disks (and dwarf spheroidals) are the most dark matter-dominated systems (Persic \& Salucci 1988, 1990; Persic et al. 1996a, 1996b; Borriello \& Salucci 2001). Such a scaling is expected if the properties of disks track those of their dark matter halos: lower mass halos are more compact (Neto et al. 2007 and references therein), and it is also well established that lower mass disks experience less efficient star formation (Bell \& de Jong 2000; Gallazzi et al. 2005). Consequently, disks and ellipticals have similar ratios $M_{\text {dyn }} / M_{*}$ at high masses $\left(\sim\right.$ a few $\left.L_{*}\right)$, but disks are more dark matter dominated [ have much higher $M_{\mathrm{dyn}}\left(R_{e}\right)$ ] at low (stellar) masses. This difference in scaling laws also relates to their stellar size-mass relations: disks obey a shallow relation $R_{e} \propto M_{*}^{0.25}$ $M_{*}^{0.35}$, roughly consistent with the scaling of halo effective radii as a function of mass, whereas spheroids obey a much steeper relation $R_{e} \propto M_{*}^{0.6}$ (Shen et al. 2003). Again, at roughly a few $L_{*}$, disks and ellipticals have similar sizes and densities, but at low masses $\left(\ll L_{*}\right)$, ellipticals are smaller (in their stellar/baryonic mass distributions) and more dense.

This difference has, for $\sim 30 \mathrm{yr}$, represented a major challenge for theory, especially models that posit that ellipticals are formed through the merger of disk galaxies (the "merger hypothesis"; Toomre \& Toomre 1972; Toomre 1977). In particular, it has been noted that purely dissipationless mergers of stellar disks cannot raise the mass and phase-space densities of ellipticals and so cannot change the scaling laws of ellipticals to something different from disks (Ostriker 1980; Carlberg 1986; Gunn 1987).

However, these arguments do not pertain if ellipticals are formed from mergers of gas-rich disks. Particularly at low masses, where ellipticals are more compact than spirals, disks have a large fraction of their mass in gas, so mergers must account for dissipation. In a merger of two disks containing both gas and stars, the stars are dissipationless and they cannot increase their phase-space density and so violently relax to a distribution with an $R_{e}$ similar to the progenitor disks. Gas, on the other hand, can radiate, and tidal torques excited during a merger can remove its angular momentum (Hernquist 1989; Barnes \& Hernquist 1991, 1996). The resulting inflows produce a dissipational, merger-induced starburst that is compact (typical size scales $\sim 0.5-1 \mathrm{kpc}$ ). If a significant fraction of the final stellar mass is formed in this manner, the scale length of the stellar component will be much smaller than that of its progenitor.

Oñorbe et al. (2005), Robertson et al. (2006b), and Dekel \& Cox (2006) argued that, because low-mass disks are more gasrich (on average) than high-mass disks, dissipation will be more important in low-mass systems. That is, lower mass ellipticals (the merger products of low-mass spirals) should have smaller effective radii relative to their progenitor disks and, since the halo mass distribution is not strongly affected by this process, a correspondingly smaller dark matter fraction (lower $M_{\mathrm{dyn}} / M_{*}$ ) within the stellar $R_{e}$. On the other hand, high-mass disks are observed to be gas-poor: at $\gg L_{*}$ gas fractions become negligible $(\ll 10 \%)$; this is precisely where ellipticals are not more compact than disks. Together, Robertson et al. (2006b) and Dekel \& Cox (2006) argued that the dependence of dissipational fraction on mass is sufficient, in principle, to explain the tilt of the FP and the sizemass and velocity dispersion-mass correlations of ellipticals.

The importance of gas dynamics and triggered star formation in mergers is reinforced by observations of ultraluminous infrared galaxies (ULIRGs; e.g., Soifer et al. 1984a, 1984b), which are invariably associated with mergers in the local universe (Joseph \& Wright 1985; Sanders \& Mirabel 1996). The infrared emission from ULIRGs is thought to be powered by intense starbursts in their nuclei, originating in central concentrations of gas (e.g., Scoville et al. 1986; Sargent et al. 1987, 1989), which will leave dense stellar remnants (Kormendy \& Sanders 1992; Hibbard \& Yun 1999; Rothberg \& Joseph 2004), as predicted theoretically (Mihos \& Hernquist 1994c). Moreover, observations of merging systems and gas-rich merger remnants (e.g., Lake \& Dressler 1986; Doyon et al. 1994; Shier \& Fischer 1998; James et al. 1999), as well as poststarburst ( $\mathrm{E}+\mathrm{A} / \mathrm{K}+\mathrm{A}$ ) galaxies (Goto 2005), have shown that their kinematic and photometric properties are consistent with them eventually evolving into typical $\sim L_{*}$ elliptical galaxies. The correlations obeyed by these mergers and remnants (e.g., Genzel et al. 2001; Rothberg \& Joseph 2006a, 2006b and references above) are similar to, e.g., the observed FP and Kormendy (1977) relations for relaxed ellipticals and consistent with evolution onto these relations as their stellar populations age, as well as the clustering and mass density of ellipticals (Hopkins et al. 2007b).

Unfortunately, the consequences of the models are less clear; as such, there has been some ambiguity regarding whether or not 
recent observations of the FP support or disagree with theory. In particular, while merger remnants may fall on the FP, it is not obvious that a differential role of dissipation is in fact responsible for the FP tilt in the manner predicted by Robertson et al. (2006b) or that this applies to all ellipticals. However, there is hope: Mihos \& Hernquist (1994c) predicted that these dissipational starbursts should leave an observable signature in the surface brightness profiles of remnants, in the form of a steep departure from the outer de Vaucouleurs (1948) $r^{1 / 4}$-law distribution in the inner regions: i.e., a central "extra light" component above the inward extrapolation of the outer profile. Observations have now uncovered distinctive evidence for this two-component structure in local ellipticals (Kormendy 1999; Kormendy et al. 2008; Ferrarese et al. 2006), classical bulges (Balcells et al. 2007), and recent merger remnants (Hibbard \& Yun 1999; Rothberg \& Joseph 2004). With the combination of Hubble Space Telescope (HST) and ground-based photometry, it now appears that such components are ubiquitous (Kormendy et al. 2008), with mass ranges and spatial extents comparable to those expected from observations of ongoing merger-induced starbursts and numerical simulations.

In a series of papers, Hopkins et al. (2008d, hereafter Paper I), Hopkins et al. (2008a, hereafter Paper II), and Hopkins et al. (2008g, hereafter Paper III), we used these simulations and data to develop and test a method to empirically determine the degree of dissipation involved in the formation of a particular elliptical galaxy, i.e., the mass fraction in the stellar remnant of a central, compact nuclear (dissipational) starburst. In Paper I we demonstrated that observed merger remnants can be robustly decomposed into two components: an outer, dissipationless (violently relaxed) component with a Sérsic law-like profile, comprising the premerger stars, and an inner, compact starburst remnant, produced in a starburst. Combining large ensembles of observations with a library of simulations that enabled us to calibrate various empirical methods, we developed a purely empirical technique to separate the inner "excess" owing to the true physical starburst component in the observed surface brightness profile from the outer profile.

In Paper II we showed that this method, given photometry of sufficient quality and covering a large dynamic range (from $\lesssim 100$ pc to $\gtrsim 20-50 \mathrm{kpc}$ ), could be extended to observed "cusp" ellipticals (i.e., ellipticals with steep nuclear profiles), commonly believed to be the direct remnants of gas-rich mergers (Faber et al. 1997). Separating the observed surface brightness profile in this manner, we demonstrated that simulations and independent observations (e.g., distinctions in stellar populations evident in kinematics, colors, stellar ages, metallicities, or abundances) confirm that the component of the elliptical formed via dissipation (in a nuclear starburst) could be reliably (statistically) determined.

In Paper III we showed that the same methods can robustly recover the dissipational starburst remnants in "core" ellipticals (ellipticals with shallow nuclear profiles). In general, even if other processes such as, e.g., scattering of stars by a binary black hole create the core, their impact on the overall starburst component is negligible (by both mass and radius, the scales of the core, typically $\lesssim 30-50 \mathrm{pc}$, are much smaller than the mass and size of the starburst). We also showed that even if core or other ellipticals have subsequently been modified by spheroid-spheroid "dry" remergers, profile shape is preserved to a sufficient degree that the original nuclear excess (i.e., the indicator of the degree of dissipation in the original, spheroid-forming merger) remains.

By applying our methodology to observations of ellipticals over a wide range in mass and size, we can, for the first time, empirically compare the degree of dissipation (starburst or dissipational mass fraction) in ellipticals to their global properties and locations on the FP. If a differential effect of dissipation as a function of mass is the explanation for the FP and elliptical scaling relations, as the models suggest, then we should be able to see and quantify the signatures of this directly in their observed profiles. Therefore, in this paper we present a critical examination of the relationship between spheroid properties and FP correlations and extra light components, in both simulations and observed galaxies. The combination of a large number of observations, together with an ensemble of hydrodynamic gas-rich merger simulations sampling the entire observed range in, e.g., mass, gas content, and other properties, enables us to develop and apply new, detailed, empirical tests of these models for the origin of the FP correlations.

In $\S 2$ we summarize our library of merger simulations, and in $\S 3$ we describe the compilation of observations used to test the models. In $\S 4$ we review different approaches to fit the surface density profile and recover the physically distinct (dissipational vs. dissipationless) components in merger remnants. We use a set of simulations to infer how galaxy properties are predicted to scale with dissipation in $\S 5$. We then compare with observed systems: examining how observed sizes and masses scale with gas content in $\S 6$, and the scaling relations obeyed at fixed dissipational content in $\S 7$. We combine the observed dependencies on dissipation and gas content in $\S 8$ to determine whether this is sufficient to explain the tilt and scatter of the FP and its projected correlations. In $\S 9$ we compare dynamical and total masses and consider possible nonhomology effects. In $\S 10$ we examine the impact of subsequent remergers on the FP correlations. Finally, in $\S 11$ we discuss our results and outline future explorations of these correlations.

Throughout we adopt a $\Omega_{M}=0.3, \Omega_{\Lambda}=0.7, H_{0}=70 \mathrm{~km} \mathrm{~s}^{-1}$ $\mathrm{Mpc}^{-1}$ cosmology and normalize all observations and models accordingly. We note that this has little effect on our conclusions, however. We also adopt a Chabrier (2003) stellar initial mass function (IMF) and convert all stellar masses and mass-to-light ratios to this choice. The exact form of the IMF systematically shifts the normalization of stellar masses herein but does not substantially influence our comparisons. All magnitudes are in the Vega system, unless otherwise specified.

\section{THE SIMULATIONS}

The merger simulations we analyze in this paper were performed with the parallel TreeSPH code GADGET-2 (Springel 2005), based on a fully conservative formulation (Springel \& Hernquist 2002) of smoothed particle hydrodynamics (SPH), which conserves energy and entropy simultaneously even when smoothing lengths evolve adaptively (see, e.g., Hernquist 1993; O'Shea et al. 2005). The simulations account for radiative cooling and optional heating by a UV background (as in Katz et al. 1996; Davé et al. 1999; although it is not important for the masses of interest here) and incorporate a subresolution model of a multiphase interstellar medium (ISM) to describe star formation and supernova feedback (Springel \& Hernquist 2003). Feedback from supernovae is captured in this subresolution model through an effective equation of state for star-forming gas, enabling us to stably evolve disks with arbitrary gas fractions (see, e.g., Springel et al. 2005; Springel \& Hernquist 2005; Robertson et al. 2006a, 2006c). This is described by the parameter $q_{\mathrm{eos}}$, which ranges from $q_{\mathrm{eos}}=0$ for an isothermal gas with effective temperature of $10^{4} \mathrm{~K}$ to $q_{\text {eos }}=1$ for our full multiphase model with an effective temperature $\sim 10^{5} \mathrm{~K}$. We also compare with a subset of simulations that adopt the star formation and feedback prescriptions from Mihos \& Hernquist (1994a, 1994c, 1996) in which the ISM is treated as a single-phase 
isothermal medium and feedback energy is deposited in a purely kinetic radial impulse (for details see, e.g., Mihos \& Hernquist 1994b).

Although we find that they make little difference to the extra light component, most of the simulations include supermassive black holes at the centers of both progenitor galaxies. The black holes are represented by "sink" particles that accrete gas at a rate $\dot{M}$ estimated from the local gas density and sound speed using an Eddington-limited prescription based on Bondi-Hoyle-Lyttleton accretion theory. The bolometric luminosity of the black hole is taken to be $L_{\mathrm{bol}}=\epsilon_{r} \dot{M} c^{2}$, where $\epsilon_{r}=0.1$ is the radiative efficiency. We assume that a small fraction (typically $\approx 5 \%$ ) of $L_{\mathrm{bol}}$ couples dynamically to the surrounding gas and that this feedback is injected into the gas as thermal energy, weighted by the SPH smoothing kernel. This fraction is a free parameter, which we determine as in Di Matteo et al. (2005) by matching the observed $M_{\mathrm{BH}}-\sigma$ relation. For now, we do not resolve the smallscale dynamics of the gas in the immediate vicinity of the black hole, but we assume that the time-averaged accretion rate can be estimated from the gas properties on the scale of our spatial resolution (roughly $\approx 20 \mathrm{pc}$, in the best cases). In any case, repeating our analysis for simulations with no black holes yields identical conclusions.

The progenitor galaxy models are described in Springel et al. (2005), and we review their properties here. For each simulation, we generate two stable, isolated disk galaxies, each with an extended dark matter halo with a Hernquist (1990) profile, motivated by cosmological simulations (Navarro et al. 1996; Busha et al. 2005), an exponential disk of gas and stars, and (optionally) a bulge. The galaxies have total masses $M_{\mathrm{vir}}=V_{\mathrm{vir}}^{3} /[10 G H(z)]$ for an initial redshift $z$, with the baryonic disk having a mass fraction $m_{d}=0.041$, the bulge (when present) having $m_{b}=0.0136$, and the rest of the mass in dark matter. The dark matter halos are assigned a concentration parameter scaled as in Robertson et al. (2006c) appropriately for the galaxy mass and redshift following Bullock et al. (2001). We have also varied the concentration in a subset of simulations and find that it has little effect on our conclusions because the central regions of the galaxy are baryon dominated. The disk scale length is computed based on an assumed spin parameter $\lambda=0.033$, chosen to be near the mode in the $\lambda$ distribution measured in simulations (Vitvitska et al. 2002), and the scale length of the bulge is set to 0.2 times this. Modulo explicit variation in these parameters, these choices ensure that the initial disks are consistent with, e.g., the observed baryonic TullyFisher relation and estimated halo galaxy mass scaling laws (Bell \& de Jong 2001; Kormendy \& Freeman 2004; Mandelbaum et al. 2006 and references therein).

Typically, each galaxy initially consists of 168,000 dark matter halo particles, 8000 bulge particles (when present), 40,000 gas and 40,000 stellar disk particles, and one black hole (BH) particle. We vary the numerical resolution, with many simulations using twice, and a subset up to 128 times, as many particles. We choose the initial seed mass of the black hole either in accord with the observed $M_{\mathrm{BH}}-\sigma$ relation or to be sufficiently small that its presence will not have an immediate dynamical effect, but we have varied the seed mass to identify any systematic dependencies. Given the particle numbers employed, the dark matter, gas, and star particles are all of roughly equal mass, and central cusps in the dark matter and bulge are reasonably well resolved. The typical gravitational softening in the simulations is $\sim 20-50 \mathrm{pc}$ in the $\lesssim L_{*}$ systems of particular interest here, with a somewhat higher $\sim 50-100 \mathrm{pc}$ in the most massive systems (yielding an effectively constant resolution $\sim 0.01 R_{e}$ in terms of the effective radius). In Papers I and II we demonstrate that this is sufficient to properly resolve not only the mass fractions but also the spatial extent of the extra light components of interest here (resolution may become an issue when attempting to model the very smallest galaxies, with $R_{e} \lesssim 100 \mathrm{pc}$ and $L<0.01 L_{*}$, but this is well below the range of the observations of interest here). The hydrodynamic gas smoothing length in the peak starburst phases of interest is always smaller than this gravitational softening.

We consider a series of several hundred simulations of colliding galaxies, described in Robertson et al. (2006b, 2006c) and Cox et al. (2006a, 2006b). We vary the numerical resolution, the orbit of the encounter (disk inclinations, pericenter separation), the masses and structural properties of the merging galaxies, initial gas fractions, halo concentrations, the parameters describing star formation and feedback from supernovae and black hole growth, and initial black hole masses.

The progenitor galaxies have virial velocities $V_{\text {vir }}=55,80$, $113,160,226,320$, and $500 \mathrm{~km} \mathrm{~s}^{-1}$ and redshifts $z=0,2,3$, and 6 , and the simulations span a range in final spheroid stellar mass $M_{*} \sim 10^{8}-10^{13} M_{\odot}$, covering essentially the entire range of the observations we consider at all redshifts, and allowing us to identify any systematic dependencies in the models. We consider initial disk gas fractions by mass of $f_{\text {gas }}=0.05,0.1,0.2,0.4,0.6$, 0.8 , and 1.0 (defined as the fraction of disk baryonic mass that is gas) for several choices of virial velocities, redshifts, and ISM equations of state. The results described in this paper are based primarily on simulations of equal-mass mergers; however, by examining a small set of simulations of unequal-mass mergers, we find that the behavior does not change dramatically for mass ratios to about $3: 1$ or $4: 1$. The mass ratios we study are appropriate for the observations of ellipticals used in this paper, which are only formed in our simulations in major merger events. At higher mass ratios, the result is a small bulge in a still disk-dominated galaxy (see, e.g., Younger et al. 2008; Hopkins et al. 2008c, 2008f), which we do not study here (although in general our conclusions should still apply, so long as the bulges of interest are "classical" bulges formed in mergers).

We also briefly consider in $\S 10$ a subset of spheroid-spheroid "remergers," representative of gas-poor or "dry" spheroid-spheroid mergers of elliptical galaxies. In these mergers, we collide two remnants of previous disk-disk mergers, in order to explore how their properties are modified through remerging. We typically merge two identical remnants (i.e., two identical copies of the remnant of a given disk-disk merger) but have also explored remergers of various mass ratios (from $1: 1$ to $\approx 4: 1$ ) and mixed morphology remergers (i.e., merging an elliptical remnant with an unmerged gas-rich disk). In the former case, we generally find a similar division in mass ratio at which a major merger is significant. In the latter, we find that the properties are more akin to those of other gas-rich (disk-disk) mergers, and the remnant should for most purposes still be considered the direct product of a gas-rich merger. In the remerger series, we vary the orbital parameters, of both the initial gas-rich merger and remerger, and consider systems with a similar range of initial gas fractions in the progenitor disks (of the original gas-rich merger), $f_{\text {gas }}=0.05,0.2,0.4$, and 0.8 . The remergers span a similar range in virial velocities and final stellar masses to the gas-rich mergers.

Each simulation is evolved until the merger is complete and the remnants are fully relaxed, typically $\sim 1-2$ Gyr after the final merger and coalescence of the BHs. We then analyze the remnants following Cox et al. (2006b) in a manner designed to mirror the methods typically used by observers. For each remnant, we project the stars onto a plane as if observed from a particular direction and consider 100 viewing angles to each remnant, which uniformly sample the unit sphere. Given the projected stellar mass 
distribution, we calculate the isodensity contours and fit ellipses to each (fitting major- and minor-axis radii and hence ellipticity at each isodensity contour), moving concentrically from $r=0$ until the entire stellar mass has been enclosed. This is designed to mimic observational isophotal fitting algorithms (e.g., Bender et al. 1987; Bender 1988). The radial deviations of the isodensity contours from the fitted ellipses are expanded in a Fourier series in the standard fashion to determine the boxiness or diskiness of each contour (the $a_{4}$ parameter). Throughout we show profiles and quote our results in terms of the major-axis radius. For further details, we refer to Cox et al. (2006b).

We directly extract the effective radius $R_{e}$ as the projected halfmass stellar effective radius and the velocity dispersion $\sigma$ as the average one-dimensional velocity dispersion within a circular aperture of radius $R_{e}$. This differs from what is sometimes adopted in the literature, where $R_{e}$ is determined from the best-fitting Sérsic profile, but because we are fitting Sérsic profiles to the observed systems, we usually quote both the true effective radius of the galaxy and effective radii of the fitted Sérsic components. Throughout, the stellar mass $M_{*}$ refers to the total stellar mass of the galaxy, and the dynamical mass $M_{\text {dyn }}$ refers to the traditional dynamical mass estimator

$$
M_{\mathrm{dyn}} \equiv k \frac{\sigma^{2} R_{e}}{G},
$$

where we adopt $k=3.8$ (roughly what is expected for a Hernquist [1990] profile, and the choice that most accurately matches the true enclosed stellar plus dark matter mass within $R_{e}$ in the simulations, although this choice is irrelevant as long as we apply it uniformly to both observations and simulations). When we plot quantities such as $R_{e}, \sigma$, and $M_{\mathrm{dyn}}$, we typically show just the median value for each simulation across all $\sim 100$ sight lines. The sight line-to-sight line variation in these quantities is typically smaller than the simulation-to-simulation scatter, but we explicitly note where it is large.

\section{THE DATA}

We compare the simulations to and test our predictions on an ensemble of observed surface brightness profiles of ellipticals, described in Papers II and III. Specifically, we consider three samples of ellipticals and a compilation of remnants of recent gas-rich mergers. The first is the $V$-band Virgo elliptical survey of Kormendy et al. (2008) based on the complete sample of Virgo galaxies down to extremely faint systems in Binggeli et al. (1985) (the same sample studied in Côté et al. 2006; Ferrarese et al. 2006). Kormendy et al. (2008) combine observations from a large number of sources (including Bender et al. 1988; R. Bender et al. 2008, in preparation; Caon et al. 1990, 1994; Davis et al. 1985; Kormendy et al. 2005; Lauer 1985; Lauer et al. 1995, 2005; Liu et al. 2005; Peletier et al. 1990) and new photometry from McDonald Observatory, the HST archive, and the SDSS for each of their objects that (after careful conversion to a single photometric standard) enables accurate surface brightness measurements over a wide dynamic range (with an estimated zero-point accuracy of $\pm 0.04 \mathrm{~V} \mathrm{mag} \operatorname{arcsec}^{-2}$ ). Typically, the galaxies in this sample have profiles spanning $\sim 12-15$ mag in surface brightness, corresponding to a range of nearly 4 orders of magnitude in physical radii from $\sim 10 \mathrm{pc}$ to $\sim 100 \mathrm{kpc}$, permitting the best simultaneous constraints on the shapes of both the outer and inner profiles of any of the objects we study. The profiles include, e.g., ellipticity, $a_{4} / a$, and $g-z$ colors as a function of radius. Unfortunately, since this is restricted to Virgo ellipticals, the number of galaxies is limited, especially at the intermediate and high end of the mass function.

We therefore add surface brightness profiles from Lauer et al. (2007b) further supplemented by Bender et al. (1988). Lauer et al. (2007b) compile $V$-band measurements of a large number of nearby systems for which HST imaging of the galactic nuclei is available. These include the Lauer et al. (2005) WFPC2 data set, the Laine et al. (2003) WFPC2 BCG sample (in which the objects are specifically selected as brightest cluster galaxies from Postman \& Lauer 1995), and the Lauer et al. (1995) and Faber et al. (1997) WFPC1 compilations. Details of the treatment of the profiles and conversion to a single standard are given in Lauer et al. (2007b). The sample includes ellipticals over a wide range of luminosities, down to $M_{B} \sim-15$, but is dominated by intermediate and giant ellipticals, with typical magnitudes $M_{B} \lesssim-18$. This therefore greatly extends our sampling of the intermediateand high-mass end of the mass function, but at the cost of some dynamic range in the data. The HST images alone, while providing information on the central regions, typically extend to only $\sim 1 \mathrm{kpc}$ outer radii, which is insufficient to fit the outer profile. Lauer et al. (2007b) therefore combine these data with groundbased measurements from a number of sources (see the references for the Kormendy et al. [2008] sample) to construct profiles that typically span physical radii from $\sim 10 \mathrm{pc}$ to $\sim 10-20 \mathrm{kpc}$. Although the composite profiles were used in Lauer et al. (2007b) to estimate effective radii, they were not actually shown in that paper. It should also be noted that there is no single criterion that characterizes galaxies included in this sample, but they generally comprise luminous nearby ellipticals and S0 galaxies for which detailed imaging is available. We emphasize that issues of completeness and, e.g., environment are not important for any of our conclusions.

We occasionally supplement the profiles from Lauer et al. (2007b) with additional profiles used in Bender et al. (1988, 1992, $1993,1994)$ and in some cases subsequently updated. These are more limited: typically the profiles cover $\sim 7 \mathrm{mag}$ in surface brightness, extending from $\sim 30-50$ pc out to roughly a few kiloparsecs (typically $\sim 3 \mathrm{kpc}$ in low-luminosity systems and $\sim 15 \mathrm{kpc}$ in the brightest systems, sufficient for acceptable, but not strong, constraints on the outer profile shapes). However, the measurements are usually in each of the $V, R$, and $I$ bands and hence allow us to construct multicolor surface brightness, ellipticity, and $a_{4} / a$ profiles. We use this to estimate, e.g., the sensitivity of the fitted parameters and galaxy profiles on the observed wave band and on the quality and dynamic range of the photometry.

We also consider the sample of local remnants of recent gasrich mergers from Rothberg \& Joseph (2004) with which we compare our simulations in Paper I. For these objects, Rothberg \& Joseph (2004) compile $K$-band imaging, surface brightness, ellipticity, and $a_{4} / a$ profiles, where the profiles typically range from $\sim 100$ pc to $\sim 10-20 \mathrm{kpc}$. These span a moderate range in luminosity (including objects from $M_{K} \sim-20$ to $M_{K} \sim-27$, but with most from $M_{K} \sim-24$ to $M_{K} \sim-26$ ) and a wide range in merger stage, from ULIRGs and (a few) unrelaxed systems to shell ellipticals. As demonstrated in Rothberg \& Joseph (2004) and argued in Paper I, these systems will almost all become (or already are, depending on the classification scheme used) typical $\sim L_{*}$ ellipticals, with appropriate phase-space densities, surface brightness profiles, FP relations, kinematics, and other properties. For a detailed discussion of the modeling of these systems and the profiles themselves, we refer to Paper I (all of the results shown for these systems are derived therein). We show the results from Paper I here in order to test whether observed merger remnants (not just our simulations) obey the same correlations 
we study in ellipticals. In particular, this allows us to provide an additional empirical check of the simulations and the argued continuity of merger and elliptical populations.

Because the generally accepted belief is that core ellipticals were not directly formed in gas-rich major mergers but were subsequently modified by dry remergers, we have repeated our analysis considering just those ellipticals that are confirmed via HST observations as being either cusp or core ellipticals and designate the two populations as two separate subsamples throughout. However, as we discuss below, treating them as a single population (or including all ellipticals, even those without HST observations, in our sample) makes no difference to our conclusions. We include all the confirmed gas-rich merger remnants but note that there are a small number of extreme unrelaxed cases for which sharp features in the surface brightness profiles prevented derivation of meaningful quantities (note, however, as shown in Paper I, that almost all of the objects in this sample are sufficiently well relaxed at the radii of interest for our fitting). We exclude spheroidals, as they are not believed to form in major mergers as are ellipticals (e.g., Kormendy 1985, 1987; Kormendy et al. 2008), and in any case they dominate at extremely low masses where our simulations do not sample the population (they also predominate as satellite galaxies, whose effects we do not model).

We also exclude $\mathrm{S} 0$ galaxies. This is not because of a physical distinction: observations suggest that these likely form a continuous family with the low-luminosity cusp ellipticals, and in fact a number of our simulated gas-rich merger remnants would, from certain viewing angles, be classified as S0s (although we exclude disk-dominated simulation remnants from our comparisons here). However, in order to derive, e.g., the parameters of the outer, violently relaxed profile and central extra light, it would be necessary to remove the contribution of the large-scale disk from the surface brightness profiles of these objects. Our two-component (outer dissipationless and inner dissipational) Sérsic models (described in $\S 4$ ) then become three-component fits, and the degeneracies involved with three independent components, even with our best data and simulations, are so large as to render the results meaningless. We have, however, revisited all of the S0s in these samples in light of our results and find that they are, in all cases, consistent with our predicted and observed trends. However, it is too difficult to infer these trends directly from the S0s themselves without ideal disk subtraction.

This yields a final sample of $\approx 180$ unique elliptical galaxies and $\approx 50$ confirmed remnants of gas-rich mergers. Most of the sample spans a range of 3 orders of magnitude in stellar mass, from $\lesssim 0.1 M_{*}$ to $\sim 10 M_{*}$, and a wide range in extra light properties. There is, of course, some overlap in the samples that define our compilation; we have $\sim 600$ surface brightness profiles for our collection of unique ellipticals, including (for many objects) repeated measurements in multiple bands and with various instruments. This turns out to be quite useful, as it provides a means to quantify error estimates in fits to these profiles. The variations between fit parameters derived from observations in different bands or made using different instruments are usually much larger than the formal statistical errors in the fits to a single profile. There are no obvious systematic effects (i.e., systematic changes in profile fits from $V$ to $I$ band), but as demonstrated in Paper I, the effects of using different bands or changing dynamic range (from different instruments) can be complex, depending on the structure and degree of relaxation of the outer regions of a system. On the other hand, there are well-relaxed objects for which almost no significant change in the fits occurs from band to band. It is therefore useful to have multiple observations of the same system, as it allows us to get some idea of how sensitive our fits are to differences in, e.g., the choice of observed wavelength or dynamic range from instrument to instrument.

In Papers II and III we present the results of our fits to each elliptical in our sample; we use these values throughout this paper. For sources with multiple independent observations, we define error bars for each fit parameter representing the $\sim 1 \sigma$ range in parameters derived from various observations, typically from three different surface brightness profiles but in some cases from as many as $\approx 5-6$ sources (where there are just two sources, the "error" is simply the range between the two fits). In many cases the different observations are comparable; in some there are clearly measurements with larger dynamic range and better resolution: the errors derived in this manner should in such cases be thought of as the typical uncertainties introduced by lower dynamic range or less accurate photometry.

In terms of direct comparison with our simulations, the data often cover a dynamic range and have resolution comparable to our simulations, provided that we do not heavily weight the very central $(\leqslant 30 \mathrm{pc})$ regions of $H S T$ nuclear profiles. Experimenting with different smoothings and imposed dynamic range limits, we find that it is unlikely that resolution or seeing differences will substantially bias our comparisons. They can introduce larger scatter, however: the robustness of our results increases considerably as the dynamic range of the observed profiles is increased.

We have converted all the observations to physical units given our adopted cosmology and compile global parameters (where not available in the original papers), including, e.g., kinematic properties, luminosities, and black hole masses from the literature. We determine stellar masses ourselves in a uniform manner for all the objects, based on their total $K$-band luminosities and $(B-V)$ color-dependent mass-to-light ratios from Bell et al. (2003) corrected for our adopted IMF. We have also repeated our analysis using stellar masses derived from a mean $M / L$ as a function of luminosity or from fitting the integrated UBVRIJHK photometry of each object to a single stellar population with the models of Bruzual \& Charlot (2003) and find that this makes no difference to our conclusions.

Throughout we usually refer interchangeably to the observed surface brightness profiles in the given bands and the surface stellar mass density profile. Of course, stellar light is not exactly the same as stellar mass, but in Papers I and II we consider the differences between the stellar light and the stellar mass density profiles as a function of time, wavelength, and properties of the merger remnant and show that the optical $(V)$ and $K$-band results introduce little bias (i.e., are good tracers of the stellar mass). It is important to note that while we are not concerned about the absolute normalization of the profile (i.e., mean $M / L$ ), since we derive total stellar masses separately from the integrated photometry, we must account for systematics that might be induced by change in $M / L$ as a function of radius. The profiles in optical bands such as $V$ require more care when the system is very young ( $\lesssim 1-2$ Gyr after the major merger-induced peak of star formation), and there can be considerable bias or uncertainty owing to stellar population gradients and dust. However, once the system is relaxed, the optical bands become good proxies for the stellar mass distribution, with $\lesssim 20 \%$ variation in our $M / L$ over the entire fitted range of radii in our simulations, in good agreement with the simple expectation based on the observed weak color gradients in most of the observed systems.

In fact, in Papers I and II we demonstrate that once the system reaches intermediate age, the bias in, e.g., $B$ or $V$ band is often less than that in $K$ band because systems tend to be both younger and more metal-rich in their centers. In $K$ band, these both increase $L / M$, leading to a (small) systematic bias. Our simulation 
results indicate that our merger remnant sample (observed in $K$ band) is, on average, robust in this sense, but they should be treated with care, especially in the most extreme cases (namely, the few LIRGs and ULIRGs in the sample). In optical bands, however, age and metallicity gradients have opposite effects (younger age increases $L / M$, but higher metallicity decreases $L / M$ ), and they tend to mostly cancel. Since essentially all of our ellipticals observed in the optical bands are older than this stellar population age (even in their centers), and they have been carefully vetted and either corrected or (where correction was too difficult) excluded for the effects of, e.g., dust lanes in the sources (Kormendy et al. 2008; Lauer et al. 2007b), we are not concerned that significant bias might persist. Furthermore, comparison of systems observed in different bands demonstrates that our conclusions are unchanged (modulo small systematic offsets) regardless of the observed bands in which we analyze these systems.

\section{INFERRING THE DEGREE OF DISSIPATION FROM OBSERVED PROFILES}

In order to test the theoretical models from Robertson et al. (2006b) and others using observed systems, we require some empirical means to estimate the amount of dissipation involved in forming elliptical galaxies. The simulations imply that the light profile should be considered to be the sum of two physically distinct components. The outer, violently relaxed, dissipationless component is made from stars that formed in the disks prior to the final stages of a merger and therefore evolve in a collisionless manner. The inner, "dissipational" component is comprised of stars produced in a central starburst, from gas that loses its angular momentum in the merger. This gas is channeled into the center of the remnant and is converted into stars on a short timescale (effectively in an almost fixed background potential set by the already nearly relaxed outer, dissipationless component). The "degree of dissipation" is, therefore, effectively measured by the mass fraction of the central, dissipational starburst component, which causes $R_{e}$ to contract and gives rise to the effects described above. In Papers I, II, and III we develop and test a methodology for decomposing observed surface brightness profiles into these two components. We refer to those papers for details and verification that this approach is reliable when applied to either simulations or observations, but we briefly review the methodology here. We consider two different observable proxies for the amount of dissipation.

First, we directly fit the surface brightness profile to determine the mass fraction in a central extra light or starburst component, which we denote as $f_{\text {extra }}$. We demonstrate in Paper I that this quantity can be measured by fitting the total observed surface brightness profile of merger remnants to the following two-component model:

$$
I_{\mathrm{tot}}=I_{\mathrm{extra}} \exp \left[-b_{1}\left(\frac{r}{r_{\mathrm{extra}}}\right)\right]+I_{o} \exp \left[-b_{n}\left(\frac{r}{r_{o}}\right)^{1 / n}\right],
$$

with the inner part $\left(I_{\text {extra }}, r_{\text {extra }}\right)$ serving as a proxy for the central dissipational component and the outer component $\left(I_{o}, r_{o}\right)$ representing the dissipationless outer profile. The extra light, in other words, is the mass fraction in a high surface brightness central component that rises above the extrapolation of the outer, intermediate $n_{s}$ Sérsic law-like profile (which in any reasonable model will be dominated by the violently relaxed stars). In Papers I and II we show that this form provides, on average, a reliable physical decomposition of the mass distribution in remnants of simulated mergers between gas-rich galaxies, including mass fractions, effective radii, and profile shapes of the two components. Analogous decompositions have been applied to, e.g., observed bulges in disk galaxies (Balcells et al. 2007) and Virgo ellipticals, motivated by purely empirical considerations including surface brightness profile shapes, isophotal shape and kinematic profiles, and central stellar populations (see Kormendy 1999; Kormendy et al. 2008 and references therein), as well as recent merger remnants (Rothberg \& Joseph 2004); we refer to Paper II for a detailed discussion and comparison with similar methodologies from the literature.

Figure 1 illustrates this procedure for two typical merger remnants from our simulation library. We show the total surface brightness profile, as well as the known physical decomposition of that profile into a prestarburst, violently relaxed component and a dissipational starburst component (here we use a cut in time about the peak starburst to identify the "starburst" stars, but as shown in Paper I, our results are not sensitive to the exact choice). We compare this with the results obtained by fitting the total profile (temporarily ignoring the known physical decomposition) to a two-component model of the form given by equation (5). In both cases, the inner component of our fit is a good proxy for the physical starburst light distribution, accurately capturing its mass fraction, effective radius, and shape where it is important, i.e., where it contributes significantly to the light profile. Likewise, the shape (Sérsic index), radius, and mass fraction of the dissipationless component are accurately recovered by this purely empirical method.

We apply this to observed merger remnants in Paper I and cusp ellipticals in Paper II and show that it yields good fits with reliable results. In particular, we find that where there is independent evidence for a distinct stellar population (i.e., a starburst superimposed on a more extended, older stellar population), this method recovers the empirically estimated starburst population. We demonstrate that other indicators, including, e.g., stellar population gradients, kinematics, and isophotal shapes, all support the physical nature of these decompositions.

In Paper III we apply this methodology to remnants of gaspoor, spheroid-spheroid remergers and show that, while the profiles are somewhat smoothed out, the general profile shape is preserved, and applying this procedure will still reliably recover the original breakdown between dissipational and dissipationless components (i.e., the starburst component formed in the original, spheroid-forming merger). We extend our analysis to a large sample of core ellipticals and similarly demonstrate the accuracy of this decomposition and agreement with stellar population models (where available); even if "scouring" by a binary black hole has flattened the profiles on $\lesssim 30-50$ pc scales, the bulk of the extra light has not been strongly affected and can still be recovered.

This emphasizes an important caveat: the merger history and series of induced dissipational events in a given galaxy may be more complex than a single or couple of idealized major mergers (see, e.g., Kobayashi 2004; Naab et al. 2007). Moreover, mergerinduced starbursts may not be the only source of dissipation; for example, stellar mass loss may replenish the gas supply and lead to new dissipational bursts (see, e.g., Ciotti \& Ostriker 2007). For our purposes, however, all dissipational star formation will appear similar when observed and have the same effects: it is convenient to simulate idealized cases, but our results should most appropriately be considered a measurement of the integrated amount of dissipation (regardless of other details of the merger and dissipational history). Experiments with, e.g., more complex merger histories, simulations of multiple simultaneous mergers, 


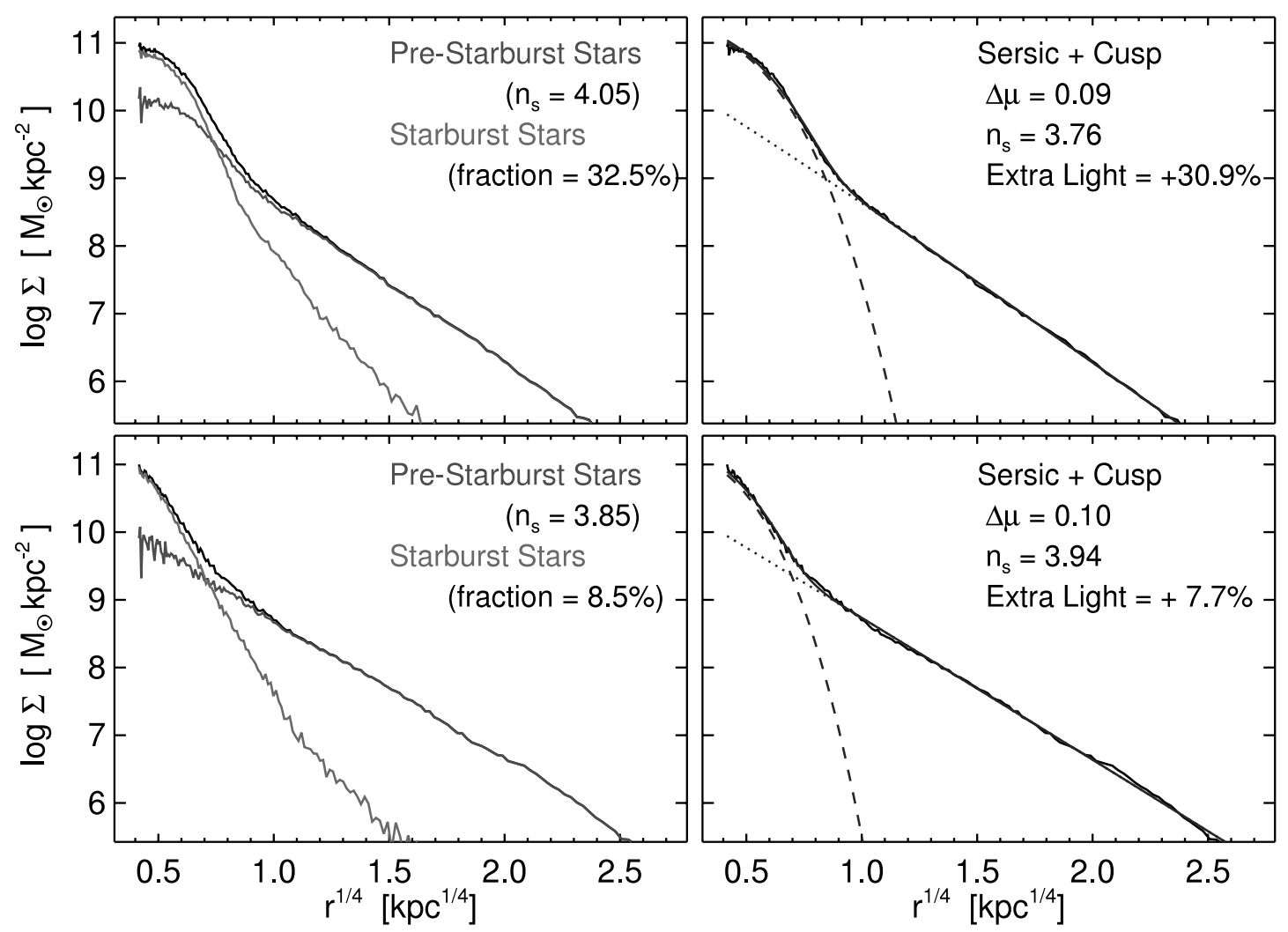

FIG. 1.-Left: Surface mass density of a typical merger remnant from our simulation library, decomposed into stars formed prior to the final merger (which are then

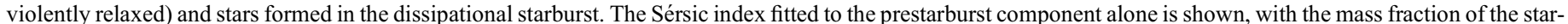

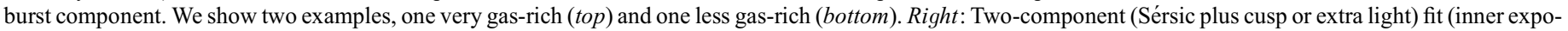

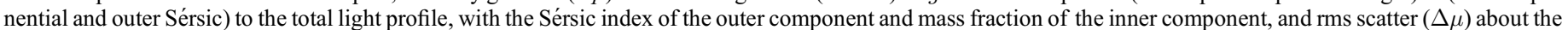

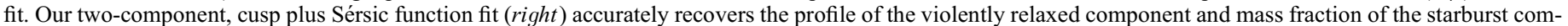
ponent. [See the electronic edition of the Journal for a color version of this figure.]

and series of dissipational events after "rejuvenation" suggest that, in this sense, our results are robust and independent of the detailed history. Furthermore, the agreement between these estimators and independent observational constraints from stellar populations, kinematics, and isophotal shapes suggest that the recovery is robust.

Second, we consider the physical starburst mass fraction $f_{\mathrm{sb}}$ implied by directly fitting simulated surface brightness profiles to the observations. In detail, we demonstrate in Papers I, II, and III that in our large library of simulations (with varying masses, gas fractions, orbital parameters, stellar and black hole feedback prescriptions, and other properties) there are remnant surface brightness profiles that (modulo small offsets in the exact normalization) agree well with the observed profiles over the entire dynamic range of the data. Considering the best-fit simulation in each case, we find that there are almost invariably simulations with similar profiles: variance less than $\Delta \mu=0.1 \mathrm{mag} \operatorname{arcsec}^{-2}$ of the simulated profile with respect to the observed profile (comparable to the inherent point-to-point scatter obtained with arbitrary spline fits to the simulated or observed profiles).

These matches are insensitive to, e.g., orbital parameters, disk initial conditions, or prescriptions for feedback and star formation (as expected given the relative independence of the inferred dissipational fractions on details of a given merger history), but good fits to a given observed profile are obtained for only a narrow range in the physical starburst mass fraction $f_{\mathrm{sb}}$ (what dependence there is on other parameters tends to indirectly reflect this: varying orbital parameters, for example, can alter the time required for a merger and therefore the mass of gas still available at the time of the final merger to participate in the central starburst). We demonstrate that we can define a robust and tightly constrained best-fit starburst mass fraction, in a $\chi^{2}$ sense, from fitting each observed profile to the entire set of simulations. These estimates agree well with the extra light mass fractions $f_{\text {extra }}$ directly determined by profile fitting and with the other indicators we consider, lending further confidence to the view that $f_{\text {extra }}$ does indeed represent a physically meaningful indicator of dissipation.

\section{THE IMPACT OF DISSIPATION: A CASE STUDY}

Robertson et al. (2006b) and Dekel \& Cox (2006) argued and Papers I and II demonstrated that increasing the quantity of gas available during the final stages of a merger (i.e., increasing the dissipative component of the merger that can collapse to small scales and form stars in a central starburst) leads to more compact remnants. Because of its importance for our analysis here, we use a subset of our simulations to highlight the physical significance of this result.

Figure 2 shows two illustrative surface density profiles of major merger remnants, the same cases shown in Figure 1. The initial galaxies were otherwise identical $\sim L_{*}$ (Milky Way-like) spirals placed on a random orbit, except that in the first example, the progenitor disks had initial gas fractions $(\sim 1.5$ Gyr before the final merger) of $20 \%$, and in the other case, of $80 \%$. In the two panels, we show the stellar mass density profile of the final (relaxed) merger remnant and separately decompose this into two physical components following Mihos \& Hernquist (1994c), namely, an outer component comprised of those stars that formed prior to 

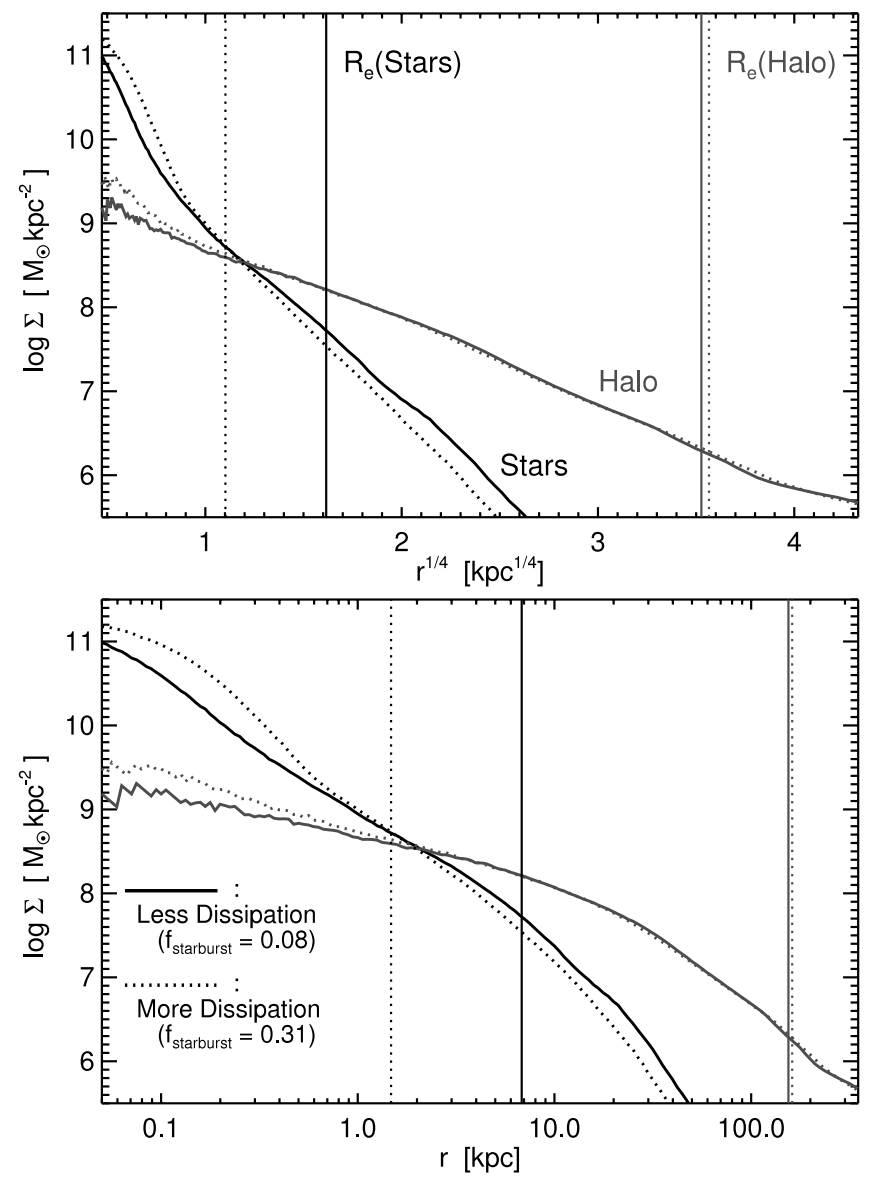

Fig. 2.- Surface density profiles of two simulated gas-rich merger remnants (the same from Fig. 1), as a function of radius $r$ (bottom) or $r^{1 / 4}$ (top). We show both the stellar profile and the dark matter (halo) profile and label the effective radii of each (vertical lines). The solid line shows a case with a moderate degree of dissipation: a mass fraction in the final, centrally concentrated and dissipational merger-induced starburst $f_{\mathrm{sb}}=0.08$. The dotted line shows a very gas-rich merger remnant, with $f_{\mathrm{sb}}=0.31$. The profile shapes are similar (the nonhomology effects are weak), and the halos are nearly identical, but the effective radii of the stellar distributions are quite different, owing to the dense central concentration of mass from the starburst in the latter case. [See the electronic edition of the Journal for a color version of this figure.]

the final coalescence of the two disks, and were therefore violently relaxed in the merger, and those stars that formed in the (by then roughly static) center of the galaxy in the final, merger-induced starburst. They correspond to $\sim 8 \%$ and $\sim 30 \%$ of the final total stellar mass in the two simulations, respectively.

In both cases, the violently relaxed stars produce an extended profile that is well described by a Sérsic law with index $n_{s} \sim 4$. Unsurprisingly, in the example with an initial gas fraction $\sim 80 \%$, the central light excess reflecting the final starburst component is much more massive; however, the profile shape is similar, and this has relatively little effect on the outer light profile (it is slightly more compact because the dense central mass concentration means that stars scattered to an orbit of a given energy will remain at slightly smaller radii in this potential, but this effect is small enough that it is not important). It is clear from Figure 1 that the differences in the two profiles primarily reflect the mass fraction of the central, dissipational component, rather than any change in the dissipationless component. We also show the surface mass density of the final dark matter halo. As expected, baryons dominate the density within the central $\sim 1-2 \mathrm{kpc}$ of the system but then trail off. Clearly, the halo mass profile is not very different in the two cases.

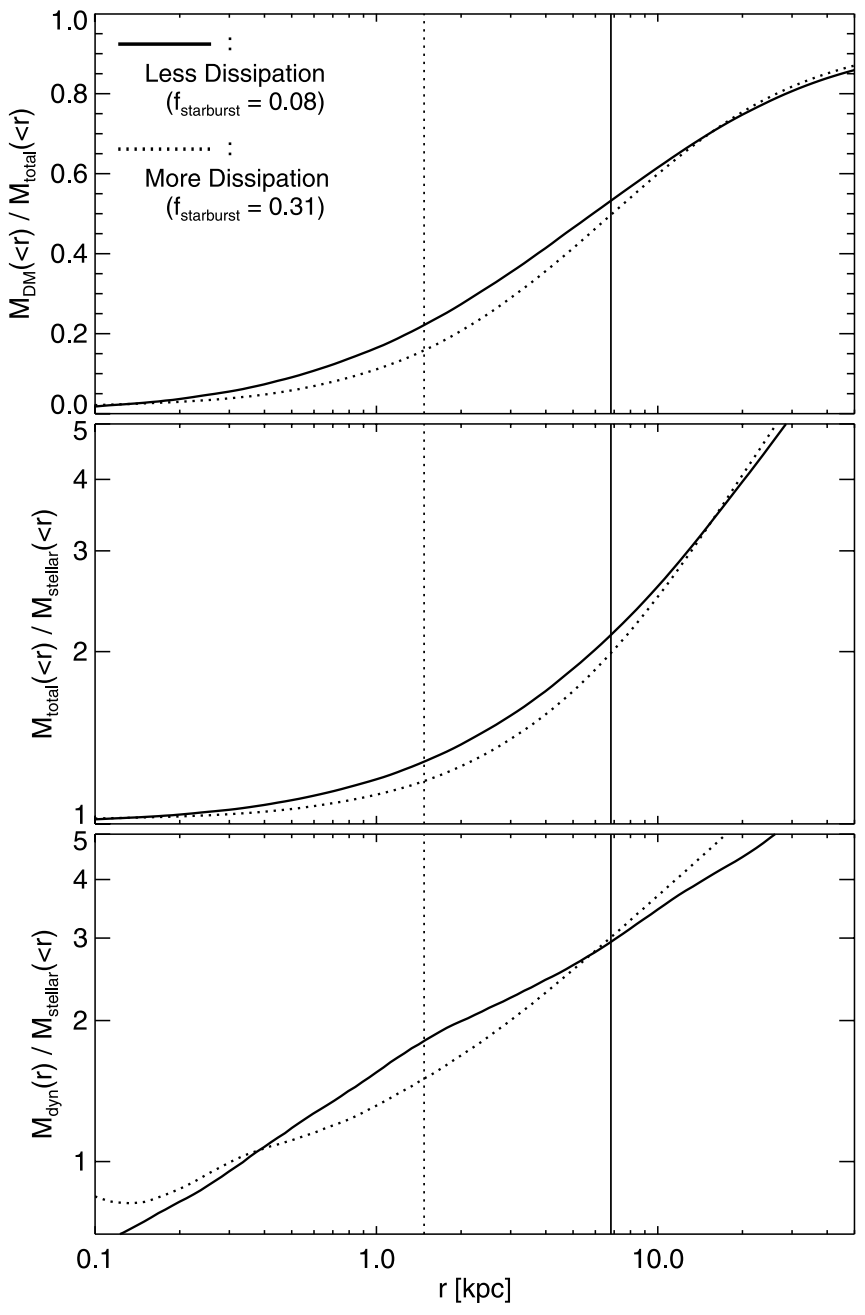

FIG. 3.-Top: Ratio of dark matter mass to total mass enclosed within the radius of the elliptical isophotal aperture with major-axis radius $r$, for the two simulations shown in Fig. 2. Vertical lines denote the effective radii of each simulation. Middle: Corresponding ratio of dark matter mass to stellar mass enclosed. Bottom: Ratio of the radius-dependent dynamical mass estimator at $r, 3.8 r \sigma(r)^{2} / G$ [where $\sigma(r)$ is measured in a narrow annulus at $r$, to the stellar mass enclosed. There is almost no difference in the scaling of the two simulations; i.e., the systems appear (in this sense) homologous, but the change in $R_{e}$ is such that evaluating these quantities at $R_{e}$ will give rather different answers for the two systems.

Despite the fact that the central mass concentration does not strongly influence the shape and size of the dissipationless remnants of the merger (both the premerger, violently relaxed stars that constitute the dissipationless stellar component of the merger and the dark matter halo), it nevertheless represents a nonnegligible component of the stellar mass, and therefore the effective radius is smaller in the case with $80 \%$ gas ( $~ 30 \%$ of its mass in the final, merger-induced compact starburst component). The resulting difference in $R_{e}$ (for such a large difference in gas fraction) is dramatic: the effective radius in the case with $f_{\mathrm{sb}}=0.08$ is $\approx 6.8 \mathrm{kpc}$, and for the case with $f_{\mathrm{sb}}=0.31$ it is $\approx 1.4 \mathrm{kpc}$. In the latter example, nearly half the mass is actually in the dissipational component, so the effective radius becomes small.

How does this effect relate to the ratio of dark matter to stellar mass? Figure 3 plots the cumulative dark matter mass fraction as a function of radius,

$$
f_{\mathrm{DM}}=\frac{M_{\mathrm{DM}}(<r)}{M_{\mathrm{DM}}(<r)+M_{*}(<r)}
$$


(for convenience we ignore the negligible mass of gas that survives the merger). We also plot the (trivially related) ratio of total enclosed mass to total enclosed stellar mass,

$$
\frac{M_{\mathrm{tot}}}{M_{*}}=\frac{M_{\mathrm{DM}}(<r)+M_{*}(<r)}{M_{*}(<r)} .
$$

It is clear that the difference as a function of radius, at least from $\sim 100 \mathrm{pc}$ to $\gtrsim 50 \mathrm{kpc}$, is not large between the two simulations (which should not be surprising: the difference in their stellar mass density profiles is primarily evident at small $r$, where a relatively small fraction of the total mass is enclosed). However, if we take the value of these ratios within the effective radii $R_{e}$ of the stellar light, we obtain two quite different results. In the gas-rich case, the large central light concentration yields a small effective radius for the stellar light, within which, for both remnants, the baryons dominate the mass. In the case with less dissipation and therefore a weaker central component and larger effective radius, the ratio is measured farther out (at the larger $R_{e}$ ), where in both simulations there are about equal masses enclosed of both dark matter and baryons.

To make a better analogy with observations, we can construct a dynamical mass estimator

$$
M_{\text {dyn }}(<r) \equiv k \sigma(r)^{2} r / G,
$$

where we adopt the factor of $k=3.8$ for convenience, as noted in $\S 2$ (making the estimator not so far from the true mass), and $\sigma(r)$ is the mean (light-weighted) projected velocity dispersion within a narrow annulus at $r$. We could also use the mean (lightweighted) dispersion within $r,\langle\sigma(<r)\rangle$, which gives a similar result (but traces the enclosed mass less faithfully). Figure 3 compares the ratio $M_{\text {dyn }} / M_{*}$ as a function of radius, which yields a similar (albeit noisier) result as considering $M_{\text {tot }} / M_{*}$. Again, it is primarily the difference in the effective radii at which this quantity is evaluated, rather than the absolute value at fixed $r$, that drives the difference between the gas-rich and gas-poor mergers.

We obtain the same result using either the true total enclosed mass or a dynamical mass estimator. This implies that there is not substantial kinematic or "traditional" structural nonhomology between our two merger remnants. We can, however, check this directly.

We compare the ratio $M_{\text {dyn }}(r) / M_{\text {tot }}(r)$ as a function of radius in Figure 4 . The quantity $M_{\text {dyn }}$ provides a good tracer of $M_{\text {tot }}$, their ratio changing by less than a factor of $\sim 2$ over 3 orders of magnitude in radius, and the ratio varies only slightly between the two simulations. The overall structure of the system has not been significantly modified, and in particular over the range of the effective radii of the two mergers, there is almost no dependence of $M_{\mathrm{dyn}}(r) / M_{\mathrm{tot}}(r)$ on either the simulation gas content or radius. In other words, the increase in the central density of the system owing to the different degrees of dissipation in the two mergers, while apparent in the nuclear profile in Figure 2, does not represent a sufficiently dramatic change in the total mass profile shape to significantly alter the virial constant [i.e., to significantly change the ratio $\left.M_{\text {true }}(r) / M_{\text {dyn }}(r)\right]$. That the estimator $M_{\text {dyn }}$ is similar across these extreme cases demonstrates that, despite the subtle difference in the strength of the central (dissipational or starburst) component in the stellar light profile, the homology assumption is valid in a purely empirical sense.

The role of dissipation in driving tilt in the FP is not, then, to introduce substantial structural nonhomology (in the sense of changing the profile sufficiently to alter the coefficient of the virial scalings) or to produce kinematic nonhomology, but primarily to

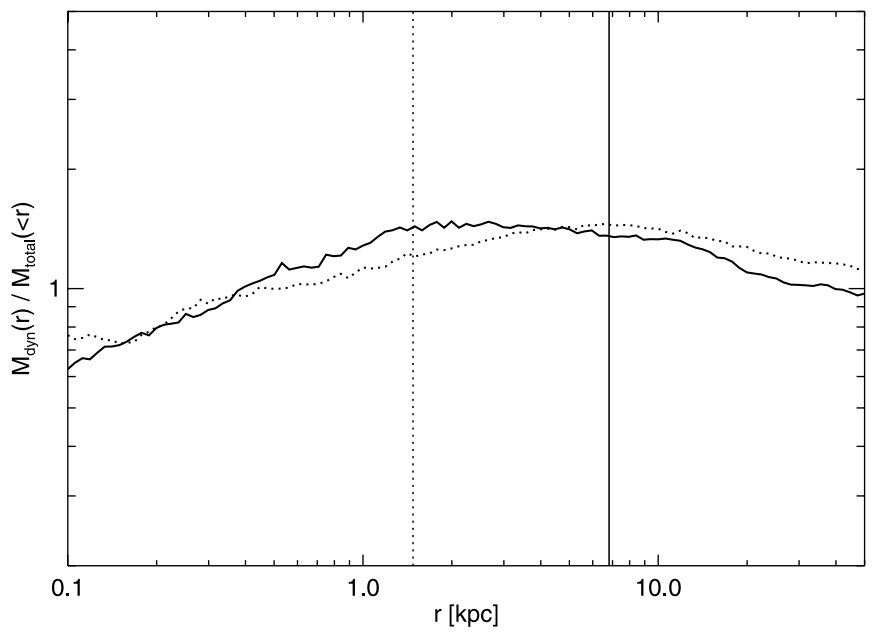

FIG. 4.- Ratio of the radius-dependent dynamical mass estimator at $r$, $3.8 r \sigma(r)^{2} / G$ [where $\sigma(r)$ is measured in a narrow annulus at $r$ ], to the true total (stellar plus dark matter) mass enclosed $\left[M_{\text {true }}(r)\right]$, for the simulations in Fig. 3 (shown in the same style; solid and dotted lines denote low and high degrees of dissipation, respectively). Vertical lines show the effective radii of each simulation. The amount of dissipation makes almost no difference in $M_{\text {dyn }} / M_{\text {true }}$ at any radius, nor is there a significant difference when evaluated at the effective radii of either system. The homology assumption that $M_{\text {dyn }} \propto M_{\text {true }}$ is valid in these simulations.

decrease the effective radius of the stellar light or mass distribution. At a smaller radius, the more stellar-dominated component of the system is sampled, and so when the traditional dynamical mass estimator is constructed $\left(M_{\mathrm{dyn}}=k \sigma^{2} R_{e} / G\right)$ and compared to the stellar mass, the ratio $M_{\mathrm{dyn}} / M_{*}$ is lower for systems with more dissipation, as argued in Robertson et al. (2006b) and Dekel \& Cox (2006). Note that here $\sigma$ is either the central velocity dispersion or the average dispersion within $R_{e}$ (it makes little difference which we consider as long as we do so uniformly, since the dispersion changes weakly with radius).

Of course, $f_{\mathrm{sb}}=0.31$ is a rather extreme case, but Figure 5 shows how the effective radius scales with gas content in another otherwise identical set of simulations of Milky Way-like spiral mergers that span a range in dissipational fraction. The effective radii decrease systematically with dissipational/starburst fraction in a continuous manner. The velocity dispersions do become slightly larger as larger central mass concentrations are assembled, but the effect is much weaker ( $\Delta \log \sigma \sim 0.15 \mathrm{dex}$, compared to $\Delta \log R_{e} \sim 0.8 \mathrm{dex}$, for $\left.f_{\mathrm{sb}}=0-0.5\right)$. The result is that the dynamical mass estimated at $R_{e}$ decreases substantially with gas fraction, again owing to the effective radius of the stellar light being drawn further in, by $\Delta \log M_{\text {dyn }} \sim 0.4$ dex from low to high gas fraction. Meanwhile, the stellar mass is almost completely unchanged, since in any case most of the gas is eventually turned into stars, whether in the premerger disks or in the final starburst. So, driven by the increasing dissipation yielding smaller effective radii, the ratio $M_{\text {dyn }} / M_{*}$ decreases by a factor $\gtrsim 2-3$ as the gas fraction increases.

\section{ASSESSING THE ROLE OF DISSIPATION IN OBSERVED SYSTEMS}

Having illustrated how dissipation can alter the sizes of stellar spheroids and the ratio of dynamical to stellar mass in simulations, we now proceed to apply our analysis to observed ellipticals.

\subsection{How Do Observed Sizes Scale with Dissipation?}

Figure 6 shows how effective radius scales with extra light mass $f_{\text {extra }}$ at fixed mass. We consider three mass bins, below, at, 

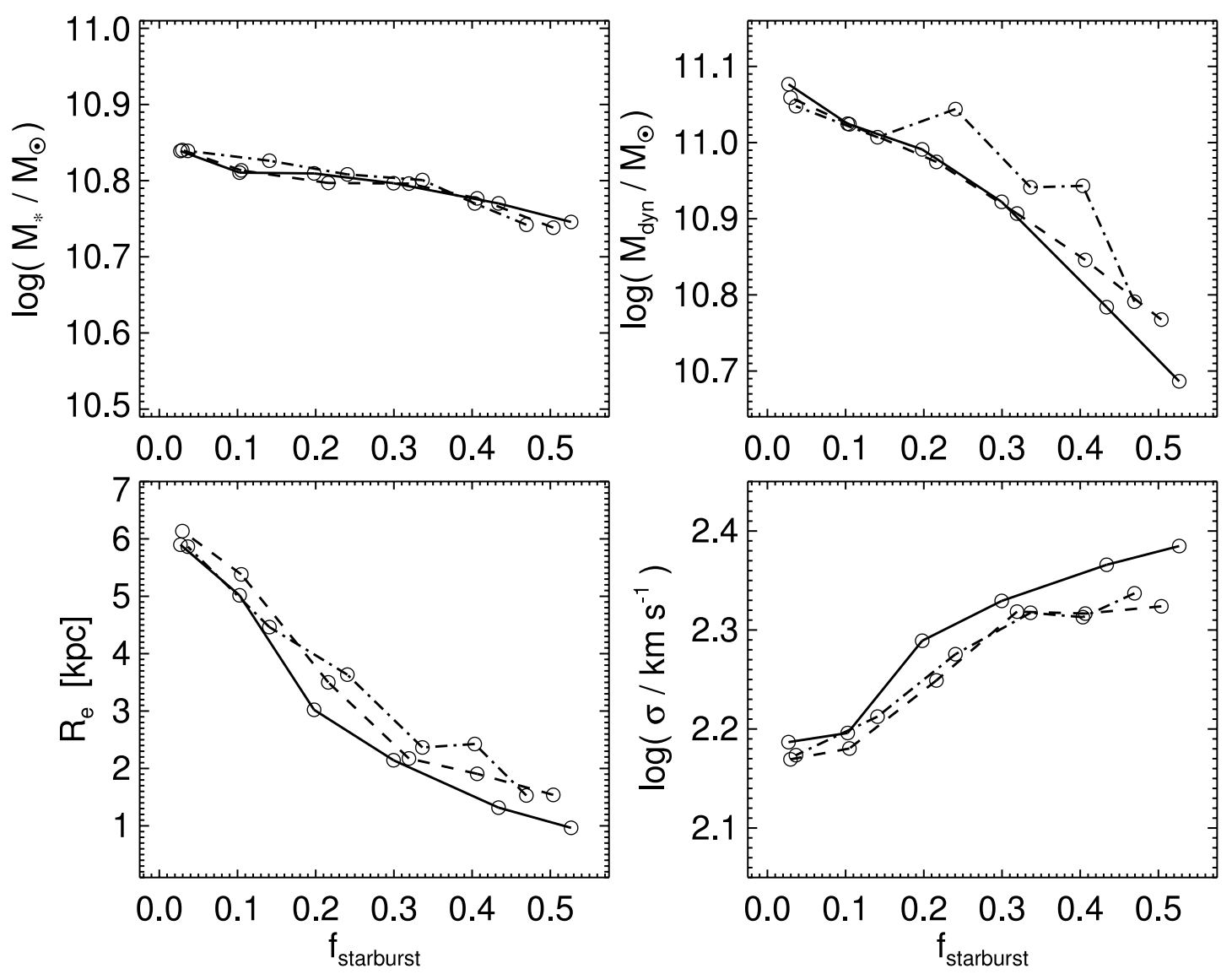

FIG. 5.- Final stellar mass, dynamical mass $M_{\text {dyn }}=k R_{e} \sigma^{2} / G$, effective radius, and central velocity dispersion as a function of the dissipational or starburst mass

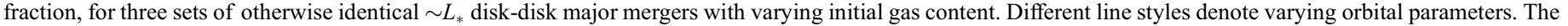

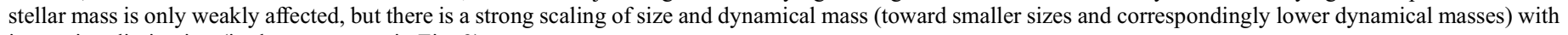
increasing dissipation (in the sense seen in Fig. 3).

and above $\sim M_{*}$. For each, we plot the residual $R_{e}$ relative to that expected for the given stellar mass, as a function of the fitted extra light fraction. Specifically, we determine $\left\langle R_{e}\left(M_{*}\right)\right\rangle$ from the sample of Shen et al. (2003) and take the ratio of the half-mass radius of each system (determined directly from the light profile, or from the fits, it does not change the comparison) to that value. Our mass bins are small enough, however, that this makes little difference compared to just, e.g., considering $R_{e}$ in a given bin. We show results separately for central cusp and core ellipticals, as well as our simulations, although the three distributions are similar.

There is a significant trend: at a given stellar mass, systems with fractionally more extra light have systematically smaller $R_{e}$ ( $\sigma$ also rises slightly, but the effect is weaker and there is more scatter at a given $f_{\text {extra }}$, as expected owing to the role of the extended stellar and dark matter distribution in setting the central potential and $\sigma$ ). In each case, the simulations and observed systems occupy a similar locus. We can also construct this plot with the starburst mass fraction $f_{\mathrm{sb}}$ of the best-fitting simulation as the independent variable (instead of the fitted extra light mass fractions $f_{\text {extra }}$ ) and find a correlation of the same nature. Given two progenitors of known size and mass, it is straightforward to predict the size of the remnant of a dissipationless merger, simply assuming energy conservation (see, e.g., Barnes 1988); in the case of a dissipative merger, Covington et al. (2008) use the impulse approximation to estimate the energy loss in the gaseous component, followed by collapse in a self-gravitating starburst. This yields a detailed approximation as a function of, e.g., initial structural and orbital parameters, but if we assume typical initial disk structural scalings and parabolic orbits, it reduces to the remarkably simple approximation

$$
R_{e} \approx \frac{R_{e}(\text { dissipationless })}{1+\left(f_{\mathrm{sb}} / f_{0}\right)}
$$

where $f_{0} \approx 0.25-0.30$ and $R_{e}$ (dissipationless) is the radius expected for a gas-free $\left(f_{\mathrm{sb}}=0\right)$ remnant. (A similar estimate is obtained by Ciotti et al. [2007] assuming, instead of the impact approximation, a constant fractional dissipational energy loss.) We plot this in Figure 6, with the scatter seen in the simulations. At all masses, in both simulated and observed cusp and core ellipticals, more dissipational ellipticals are smaller in the manner predicted.

This result directly implies that a structural difference, albeit not traditional structural nonhomology, plays a key role in establishing the FP tilt. At fixed mass, smaller systems are so because a larger fraction of their mass is formed in a central dissipational starburst (to the extent that our extra light fractions recover this dissipational component). This dissipational starburst is compact, so even though the preexisting stars are distributed to large radii, the effective radius is smaller. In Paper II we considered the light profiles of observed systems along the $R_{e}-f_{\text {extra }}$ correlation, at fixed stellar mass: it is clear that observed systems at fixed mass with the largest $R_{e}$ show profiles close to a pure Sérsic law, with little evidence for any extra light component at $>0.01 R_{e}$ (indeed, they have $f_{\mathrm{sb}} \lesssim 0.02$ ). Observed systems in this regime 


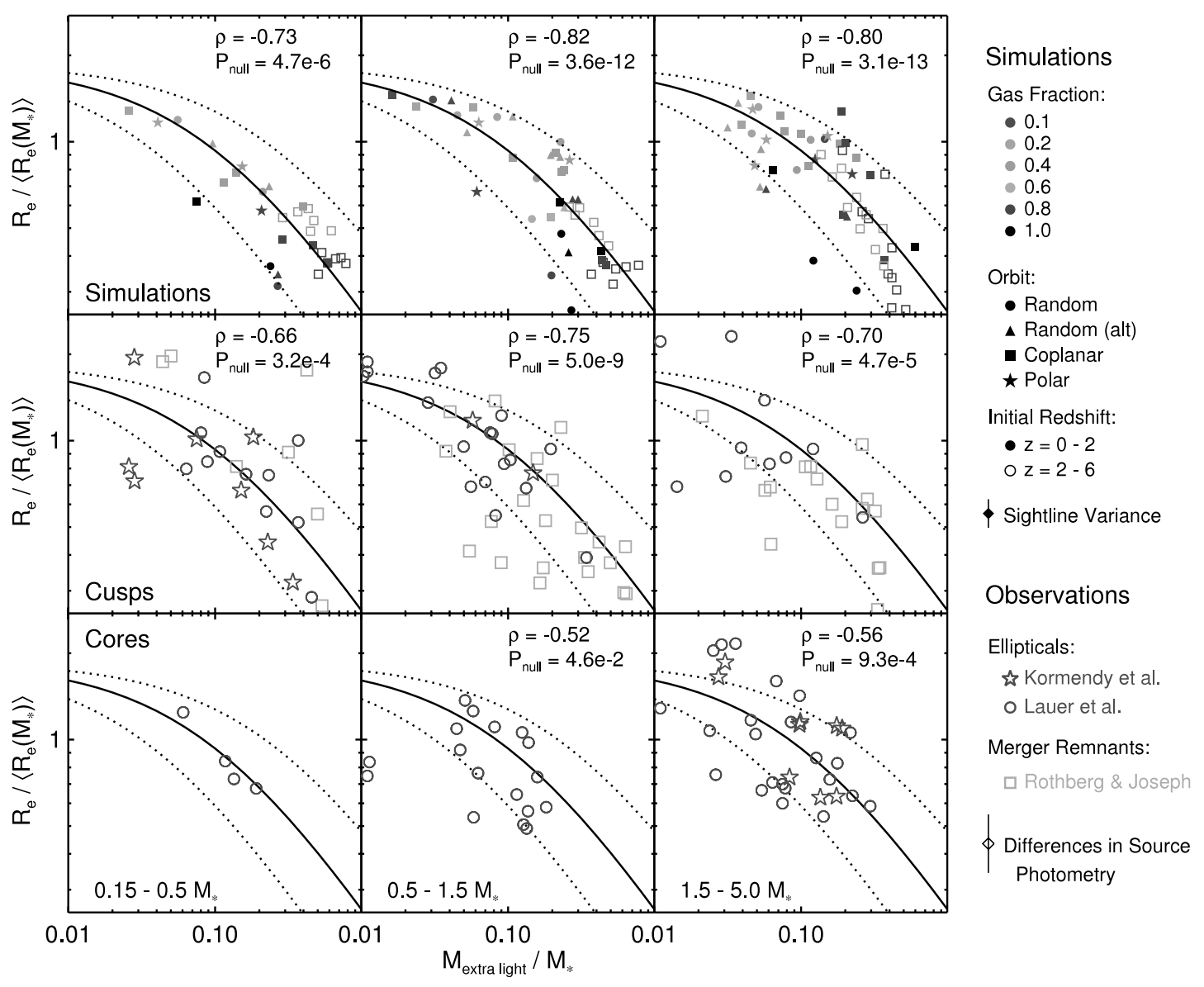

Fig. 6.-Effective radius $R_{e}$ relative to the median value for all ellipticals of the same stellar mass, as a function of our fitted extra light fractions (the empirical tracer of the dissipational/starburst mass fraction). We show simulated gas-rich merger remnants (top), observed cusp ellipticals and gas-rich merger remnants (middle), and observed core ellipticals (bottom); we use this point notation (key) throughout. We show this in 3 bins of stellar mass (relative to $M_{*} \approx 10^{11} M_{\odot}$, or $M_{V}^{*}=-21$ ). Solid (dashed) lines show the mean $( \pm 1 \sigma)$ correlation, following the analytic solution for dissipational mergers and fits to our simulation in Covington et al. (2008). We show the Spearman rank correlation coefficient and probability of the null hypothesis $P_{\text {null }}$ (no correlation) in each panel. Simulations and observations exhibit the same behavior: systems with smaller $R_{e}$ at fixed mass have systematically larger extra light fractions (in the sense predicted by Fig. 5 for the starburst/dissipational mass fractions). This implies that, at fixed mass, systems are driven along the FP by the relative amount of dissipation involved in their formation. This behavior is true regardless of cusp/core status. [See the electronic edition of the Journal for a color version of this figure.]

can still be classified as "cusp" ellipticals, but the cusps tend to be prominent at small radii and (in several cases) somewhat shallow, and they contribute negligibly to the stellar mass. However, moving to smaller $R_{e}$ and higher $f_{\text {extra }}$ at fixed stellar mass, deviations from a Sérsic law at $r \ll R_{e}$ become more noticeable. This is not to say that these deviations are universal (that the extra light always takes the same shape/form), but there are increasingly prominent central light concentrations. If the systems were perfectly homologous (in the strict sense of the definition), there should be no differences, and there should be no trend whatsoever in Figure 6.

Figure 6 also shows that there is little difference, in this sense, between the size scalings with dissipation of cusp and core ellipticals. We demonstrate this in Paper III, in both simulations and observations. In short, this is expected: even if the systems with cores have expanded via remergers, they should (so long as there is not a wide range in number of remergers or strong systematic dependence of the number of remergers on starburst fraction at fixed mass) grow uniformly and preserve these trends. We might expect some normalization offset: if the mean size-mass relation after a gas-rich merger is a power law $R_{e} \propto M_{*}^{\alpha}$, and two such systems with mass ratio $f$ (where $f$ is the mass ratio of the secondary to the primary) are involved in a dry merger from a parabolic orbit (and preserve profile shape), then the remnant will increase in radius by a factor $(1+f)^{2} /\left(1+f^{2-\alpha}\right)$ relative to the primary. However, it has also grown in mass, so compared to ellipticals of the same (final) mass, its relative increase in size is only $(1+$ $f)^{2-\alpha} /\left(1+f^{2-\alpha}\right)$. For observationally suggested values $\alpha=0.56$ (Shen et al. 2003), this predicts a $\sim 30 \%(\sim 0.1$ dex) relative size increase for major remergers with mass ratios of $1: 3$ through $1: 1$. This is easily dwarfed by the effects of dissipation seen in Figure 6, which can alter the sizes of systems by nearly an order of magnitude at fixed mass. That there is not a substantial normalization offset between the trends for cusp and core ellipticals in Figure 6 therefore implies that typical core ellipticals have been involved in only a modest number of remergers (see also Ciotti et al. 2007), but it is not a strong constraint. The important point is that observations demonstrate that the dominant factor controlling the sizes of ellipticals is the amount of dissipation, even for systems that have undergone remergers.

\subsection{How Does This Change Spheroid Dynamical Masses?}

Figure 7 plots the quantity $M_{\mathrm{dyn}} / M_{*}$ as a function of the amount of dissipation, quantified by $f_{\text {extra }}$. The expected trend based on our fiducial cases in $\S 5$ and the dissipational models (Oñorbe et al. 2005; Robertson et al. 2006b; Dekel \& Cox 2006) is borne 


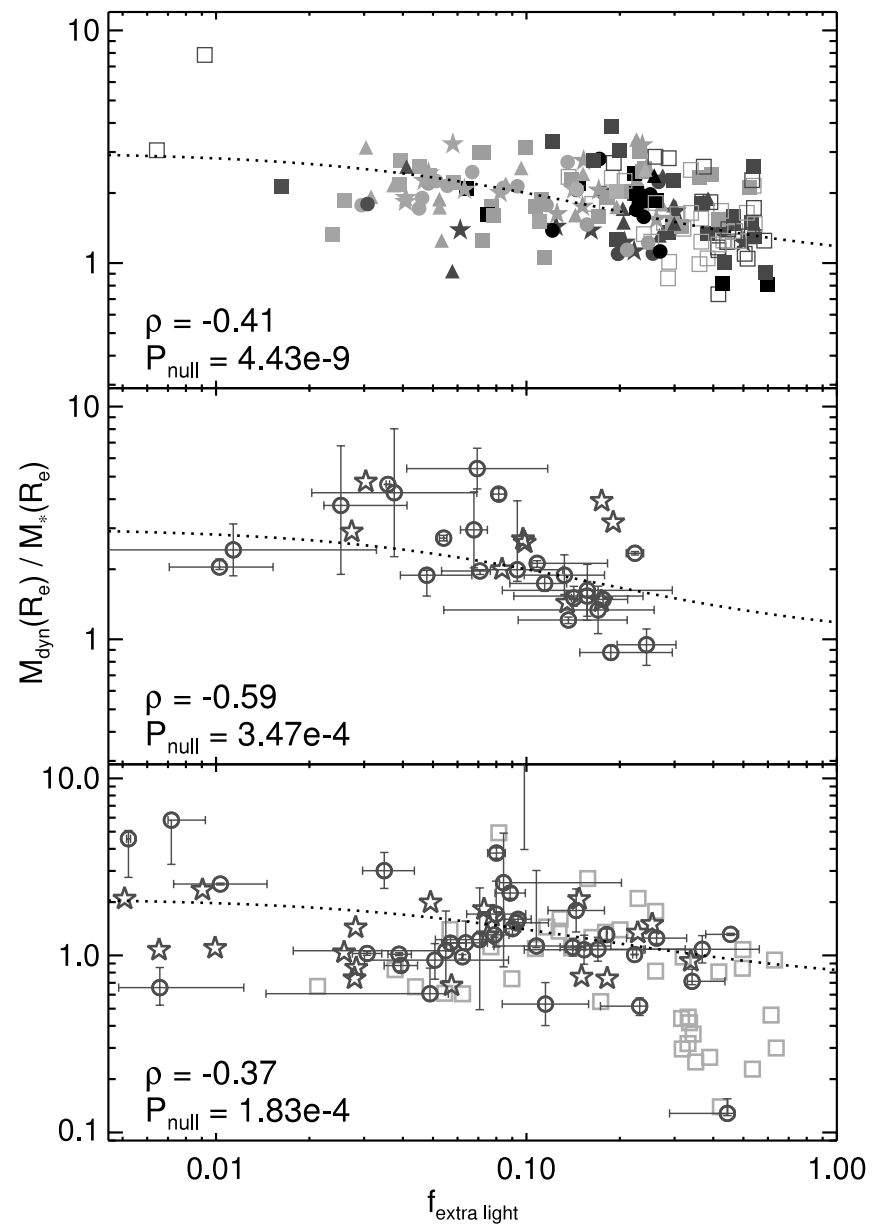

FIG. 7.- Ratio of dynamical to stellar mass within $R_{e}$, as a function of extra light fraction derived from surface brightness profiles. Results are shown for simulated gas-rich merger remnants (top), cusp ellipticals and gas-rich merger remnants (middle), and core ellipticals (bottom). The dotted line shows an approximation to the mean simulation trend. We show the Spearman rank correlation coefficient $(\rho)$ for each subsample and the probability $P_{\text {null }}$ of the null hypothesis that there is no correlation between $M_{\mathrm{dyn}} / M_{*}$ and $f_{\text {extra }}$. Systems with larger degrees of dissipation ( larger $f_{\text {extra }}$ ) have larger ratios $M_{\text {dyn }} / M_{*}$, with high significance, in the sense expected from Fig. 5. [See the electronic edition of the Journal for a color version of this figure.] out in our library of simulations over a wide dynamic range with fairly small scatter, despite a wide range of total masses, orbital parameters, initial gas fractions, assumptions regarding the gas pressurization, and other progenitor properties. The same trend obtains for the observed systems, whether cusp or core ellipticals. The scatter is significantly larger among the observed systems, but this is not surprising, given both the measurement errors involved and the possibility for more complex growth histories that our simulations do not completely model. In a formal sense, the inverse correlation (i.e., smaller $M_{\mathrm{dyn}} / M_{*}$ at larger dissipational fraction $f_{\text {extra }}$ ) is highly significant: we plot the Spearman rank correlation coefficient $\rho$ of each subsample, together with the significance of its deviation from zero. This $P_{\text {null }}$ represents the probability of the null hypothesis of no correlation between $M_{\text {dyn }} / M_{*}$ and $f_{\text {extra }}$, which is what would be expected if systems were perfectly homologous or if extra light did not contract systems and yield smaller $R_{e}$.

It is possible, in principle, that the observed correlation could be indirect, for example, if lower mass systems happened to have higher $f_{\text {extra }}$, but it had no causal connection to their lower $M_{\text {dyn }} / M_{*}$. In Figure 8 we therefore consider this same comparison in narrow bins of stellar mass. We note that, while there is still some width to our bins, they are sufficiently narrow that we obtain the same answer if we assume that all systems in the bin have the same stellar mass. We also check a series of Monte Carlo experiments where we assume that $M_{\mathrm{dyn}} / M_{*}$ and $f_{\text {extra }}$ are both pure functions of $M_{\text {dyn }}$ or $M_{*}$ but have no dependence on each other, and we find that the mass bins plotted in Figure 8 are sufficiently narrow that if this null hypothesis were true, then the maximal indirect correlation would have a significance of only $\rho \sim-0.1$ to -0.3 (with $P_{\text {null }} \approx 0.2-0.8$ ); so a significance $P_{\text {null }} \ll 0.1$ is indeed meaningful. We caution that at a narrow range in stellar mass, there is naturally less observed dynamic range in dissipation fraction (as expected, since, e.g., disks of a given mass have a reasonably narrow range of observed gas fractions), and the number of objects in each bin is smaller, so the significance of the inverse correlation in any one bin will be correspondingly reduced. However, we still see an inverse correlation in all bins, with significance stronger than expected from the null hypothesis. Near $\sim M_{*}$ where the sample size is large, the inverse correlation is still highly significant. Further, while the significance in any one of the plotted bins may be lower owing to the binning, the cumulative significance

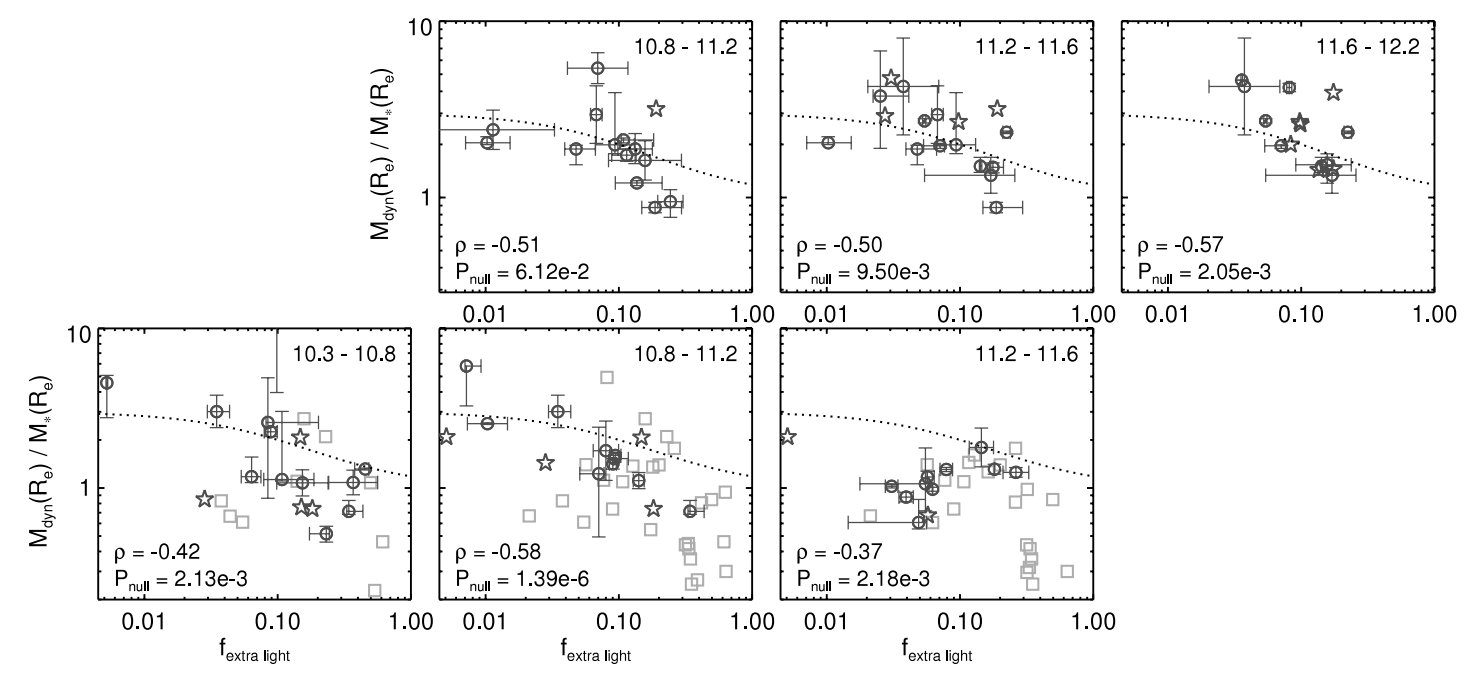

FIG. 8.- Same as Fig. 5, but in narrow bins of stellar mass (range in $M_{*}$ for each bin shown in the upper right corner of each panel). Cusp ( bottom) and core (top)

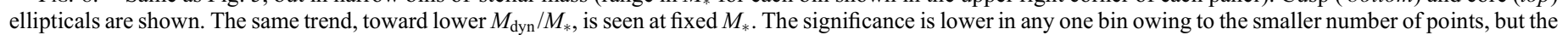
cumulative significance is comparable to or higher than that in Fig. 7. [See the electronic edition of the Journal for a color version of this figure.] 


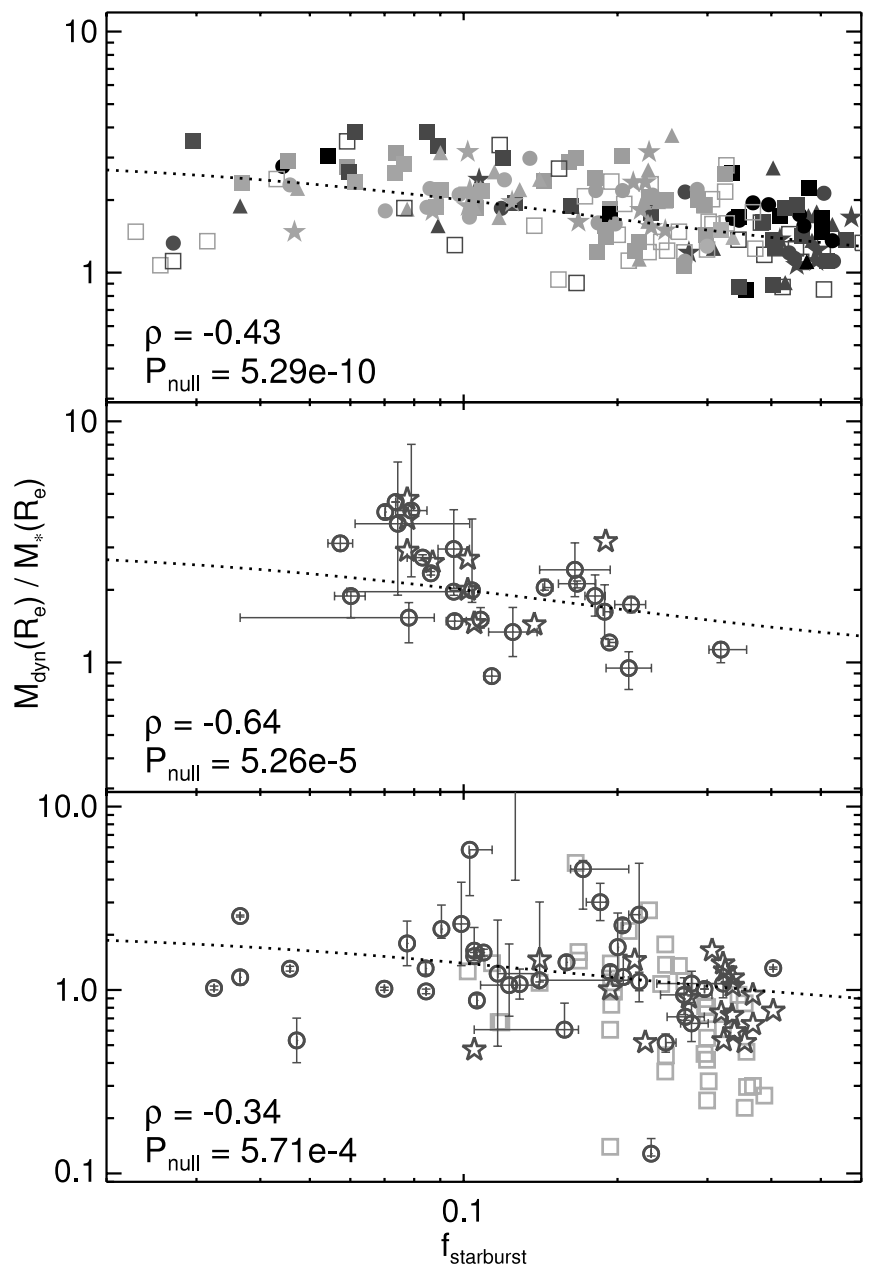

FIG. 9.- Same as Fig. 7, but using the starburst mass fraction $f_{\mathrm{sb}}$ (the physical mass fraction in our simulations, or that inferred from directly fitting simulations to the observed light profiles for the observations) instead of the purely empirical $f_{\text {extra }}$ as our estimator of the dissipational mass fraction. The results are similar. [See the electronic edition of the Journal for a color version of this figure.] (i.e., likelihood of obtaining such consistent inverse correlations, considering each mass bin as an independent subsample) is actually quite high $\left(P_{\text {null }} \ll 10^{-6}\right)$.

Figures 9 and 10 repeat this test, using the starburst mass fraction $f_{\mathrm{sb}}$ estimated from fitting simulations to observed light profiles as the proxy for the dissipational fraction. We obtain similar answers to our previous comparison using the fitted $f_{\text {extra }}$, suggesting that our comparison is not peculiar to the exact estimator used, so long as it robustly recovers the physical dissipational component of the galaxy. Again, the cumulative significance of the inverse correlation, namely, that $M_{\mathrm{dyn}} / M_{*}$ is smaller with increasing $f_{\mathrm{sb}}$ at fixed mass, is very high, $P_{\text {null }} \sim 10^{-8}$.

\section{IS DISSIPATION NECESSARY TO EXPLAIN THE FP?}

These comparisons, while demonstrating that the dissipational mass fraction does indeed correlate with the sizes and ratio $M_{\mathrm{dyn}} / M_{*}$ in observed ellipticals (and therefore that it could be the source of the tilt in the FP), do not necessarily indicate how much of the FP tilt derives from dissipation. To test this, we construct the FP in Figure 11, specifically the correlation between $M_{\text {dyn }}$ and $M_{*}$, in bins of similar fitted extra light mass fractions $f_{\text {extra }}$ (i.e., we consider the FP for systems only with similar amounts of dissipation). Because we are binning by extra light mass and still attempting to fit a correlation, we include both cusp and core ellipticals in the observed sample. Separating the two gives identical results, but the significance is reduced owing to the limited dynamic range from further splitting the sample at fixed $f_{\text {extra }}$. We fit a power law to the data (and separately, to our simulations) in each bin, of the form $M_{\text {dyn }} \propto M_{*}^{1+\alpha}$ (eq. [1]), where $\alpha$ is the FP "tilt." Because neither $M_{\text {dyn }}$ nor $M_{*}$ should properly be considered an independent variable, we quote the results from fitting the least-squares bisector to the correlation. However, this makes little difference [the least-squares best-fit $M_{\text {dyn }}\left(M_{*}\right)$ and $M_{*}\left(M_{\text {dyn }}\right)$ relations are both consistent and agree]. We show this using the typical estimator $M_{\text {dyn }}$ here but have also considered the true total enclosed mass $M_{\text {true }}$ within $R_{e}$ and find identical results (as expected, since we show in $\S \S 5$ and 9 that $M_{\text {dyn }}$ is a good proxy for $\left.M_{\text {true }}\right)$.

The results are unambiguous in both our simulations and the observed systems. The tilt in any given bin is negligible, within $1 \sigma$ of $\alpha=0$ in every case. In other words, at fixed $f_{\text {extra }}$, we recover the virial correlation with constant $M_{\mathrm{dyn}} / M_{*}$. Note that
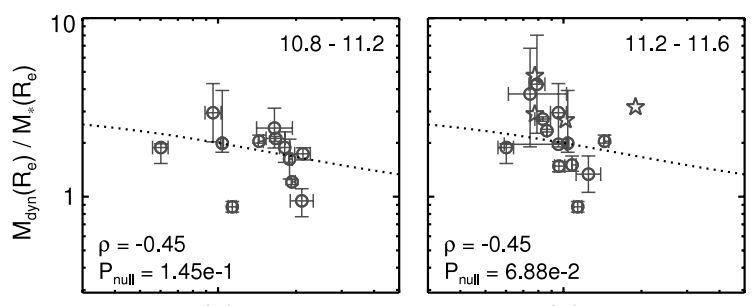

0.1
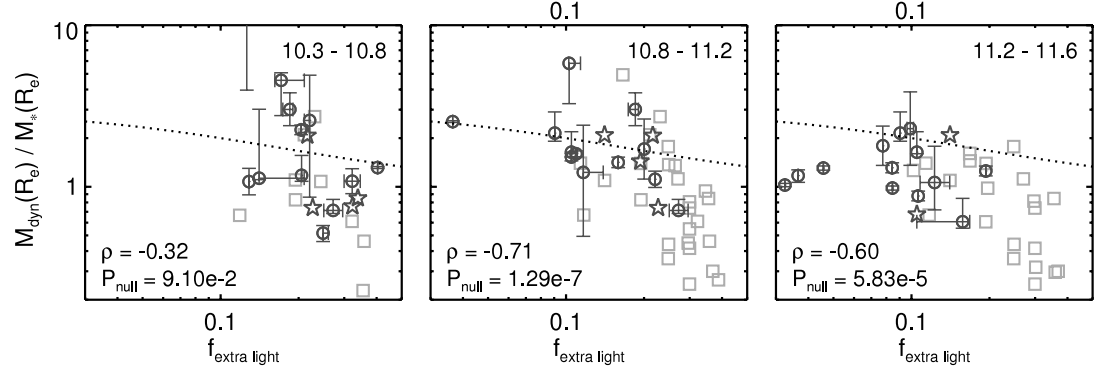

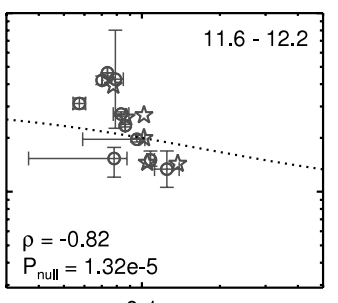

0.1

Fig. 10.- Same as Fig. 8, but again using the physical starburst mass fraction $f_{\mathrm{sb}}$ instead of the fitted extra light mass fraction $f_{\text {extra }}$. The results are similar in either case. [See the electronic edition of the Journal for a color version of this figure.] 

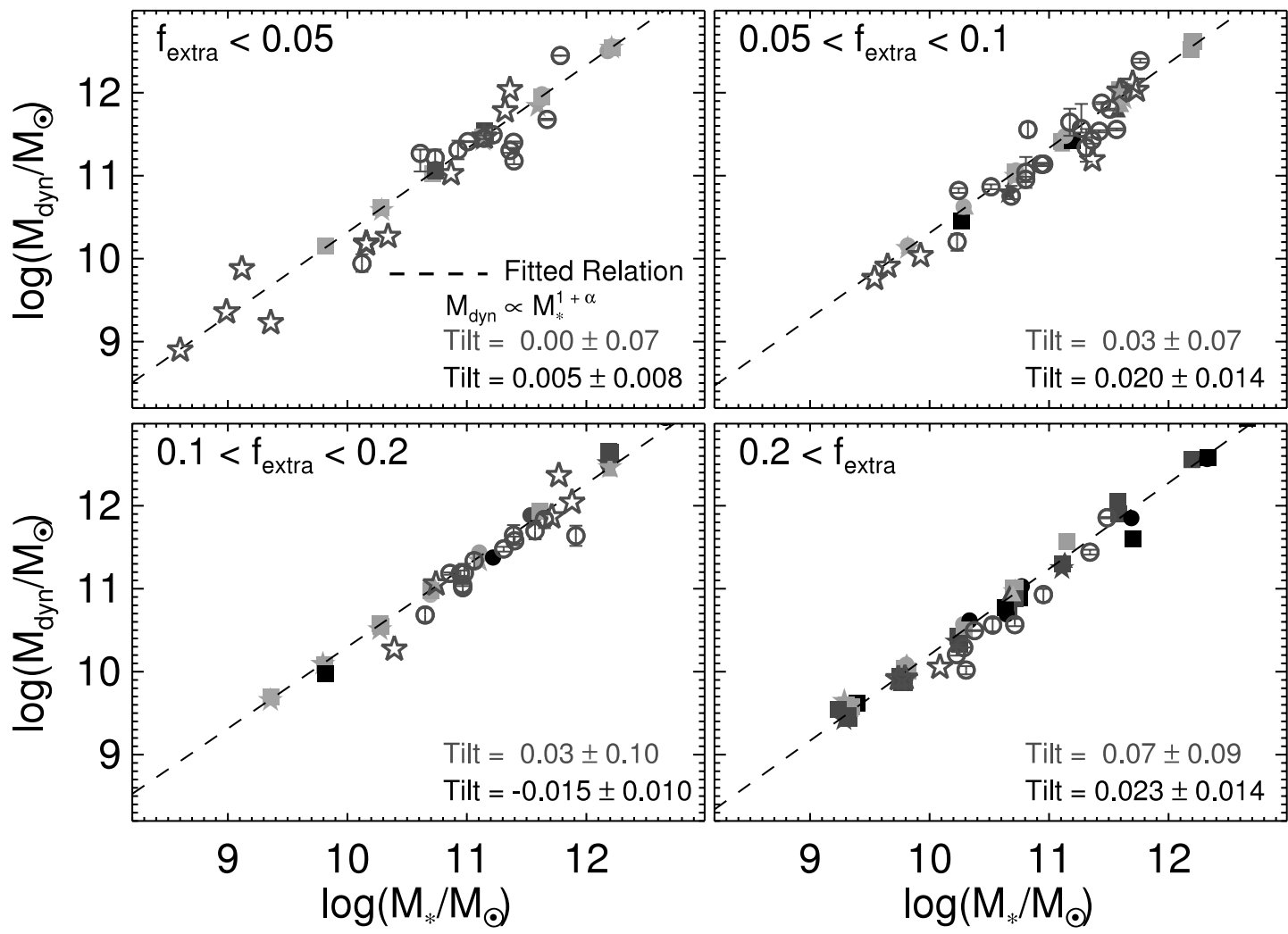

FIG. 11.-Stellar-mass FP (i.e., $M_{\mathrm{dyn}}-M_{*}$ correlation), for simulated merger remnants (black filled symbols) and observed ellipticals (open gray symbols), restricted to systems with a narrow range of extra light/dissipational mass fractions $f_{\text {extra }}$ (as labeled). In each bin of $f_{\text {extra }}$, we fit the simulations and observations separately to a correlation of the form $M_{\mathrm{dyn}} \propto M_{*}^{1+\alpha}$, where $\alpha$ quantifies the FP tilt. The resulting tilts are shown. The tilt is consistent with zero in every bin and is lower than the expected tilt of the entire population $(\alpha \sim 0.2)$ by $\sim 3 \sigma$; i.e., there is no tilt in either observed or simulated populations at the same, fixed value of $f_{\text {extra. }}$. [See the electronic edition of the Journal for a color version of this figure.]

in several of these bins the dynamic range in mass is still large ( $\sim 2-3$ orders of magnitude in $M_{*}$ ), and the number of observed systems is sufficiently large that if a substantial tilt like that observed for the entire population $(\alpha \sim 0.2)$ were present in the bin, we should see it. This is reflected in the quoted errors, which show that the tilt in most bins is inconsistent with that observed for the global population at $\sim 3 \sigma$ significance (in the sense that it is much smaller, consistent with a pure virial correlation). If we combine the data (i.e., normalize out the mean $M_{\mathrm{dyn}} / M_{*}$ at each bin in $f_{\text {extra }}$ and fit, or take the cumulative significance of the bins shown), we obtain $\alpha=0.028 \pm 0.040$, consistent with zero and $\sim 4-5 \sigma$ below the observed tilt for the entire population.

Figure 12 repeats this exercise, using the starburst mass fraction $f_{\mathrm{sb}}$ (estimated from the physical starburst mass fractions in the best-fitting simulations) as the proxy for dissipational mass fraction, instead of the fitted extra light fraction $f_{\text {extra }}$. In either case we reach identical conclusions for both the simulations and observations. The inferred tilt at fixed dissipational fraction is always consistent with zero, in some cases scattering to $\alpha<0$ (i.e., the opposite sense of what is observed; but again these are all consistent with $\alpha=0$ ). In short, we have demonstrated empirically that without invoking some systematic dependence of dissipational fraction on mass, there is no FP tilt. Dissipation is observationally necessary to explain the FP tilt.

Figure 13 also shows this for the effective radius projection of the FP. We obtain identical answers in this case (for both simulations and observations) but note that the sense of the correlation is such that, if the baseline in $R_{e}$ is comparable to the scatter in observed points, severe biases can be introduced. We see this reflected in the fact that, in this case, fitting $R_{e}\left(R_{\text {pred }}\right)$ versus
$R_{\text {pred }}\left(R_{e}\right)$ (where $R_{\text {pred }} \propto \sigma^{2} I_{e}^{-1}$ is the virial expectation) yields rather different slopes. Therefore, while we show our simulations (which have small dispersion in $R_{e}$ across sight lines), we refrain from fitting the observations in such narrow bins in this particular projection of the FP.

\section{PREDICTING THE FP: IS DISSIPATION SUFFICIENT?}

\subsection{Systematic Dependence of Dissipation on Mass: What Is Expected and Observed}

We have shown that $M_{\mathrm{dyn}} / M_{*}$ depends on the degree of dissipation, reflected in $f_{\text {extra }}$ or $f_{\mathrm{sb}}$, and that at fixed $f_{\text {extra }}$ or $f_{\mathrm{sb}}$ there is essentially no FP tilt. This already implies that dissipation must be responsible for the majority of the tilt. But if we knew how much dissipation was expected as a function of galaxy mass (i.e., the mean expected mass fraction in a dissipational starburst, for mergers of systems of a given mass), we could entirely predict the FP and its tilt.

Figure 14 shows the mean dissipational mass fractions of both cusp and core ellipticals, as a function of mass, derived in Papers II and III. There is a clear systematic trend (discussed in those papers): lower mass systems have systematically higher dissipational mass fractions. To the extent that $M_{\mathrm{dyn}} / M_{*}$ depends on dissipational fraction $\left(f_{\text {extra }}\right.$ or $\left.f_{\mathrm{sb}}\right)$, then, the existence of a systematic dependence of $f_{\text {extra }}$ and $f_{\text {sb }}$ on mass will yield a systematic dependence of $M_{\mathrm{dyn}} / M_{*}$ on mass. We can use the observed dependence of the dissipational mass fraction on mass to predict and empirically estimate the amount of tilt in the FP contributed by systematic trends in dissipation as a function of mass. For convenience, we noted in Paper II that the dependence of dissipational 

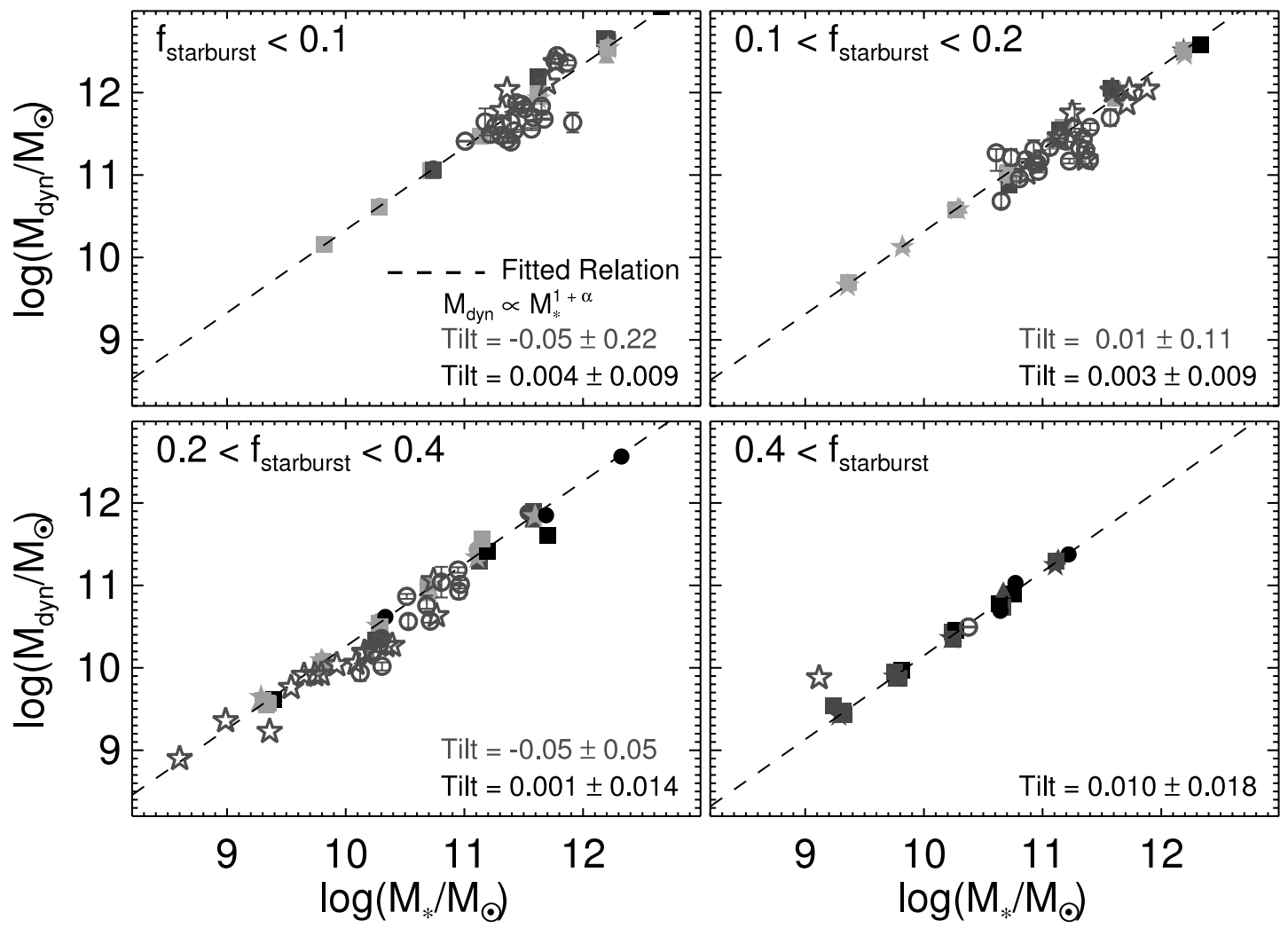

Fig. 12.- Same as Fig. 11, but again using the physical starburst mass fraction $f_{\mathrm{sb}}$ instead of the fitted extra light mass fraction $f_{\text {extra }}$. The results are similar in either case: there is a highly significant lack of tilt at fixed dissipational fraction, despite the observations and simulations still spanning a large baseline $(\sim 2$ dex $)$ in mass. [See the electronic edition of the Journal for a color version of this figure.]
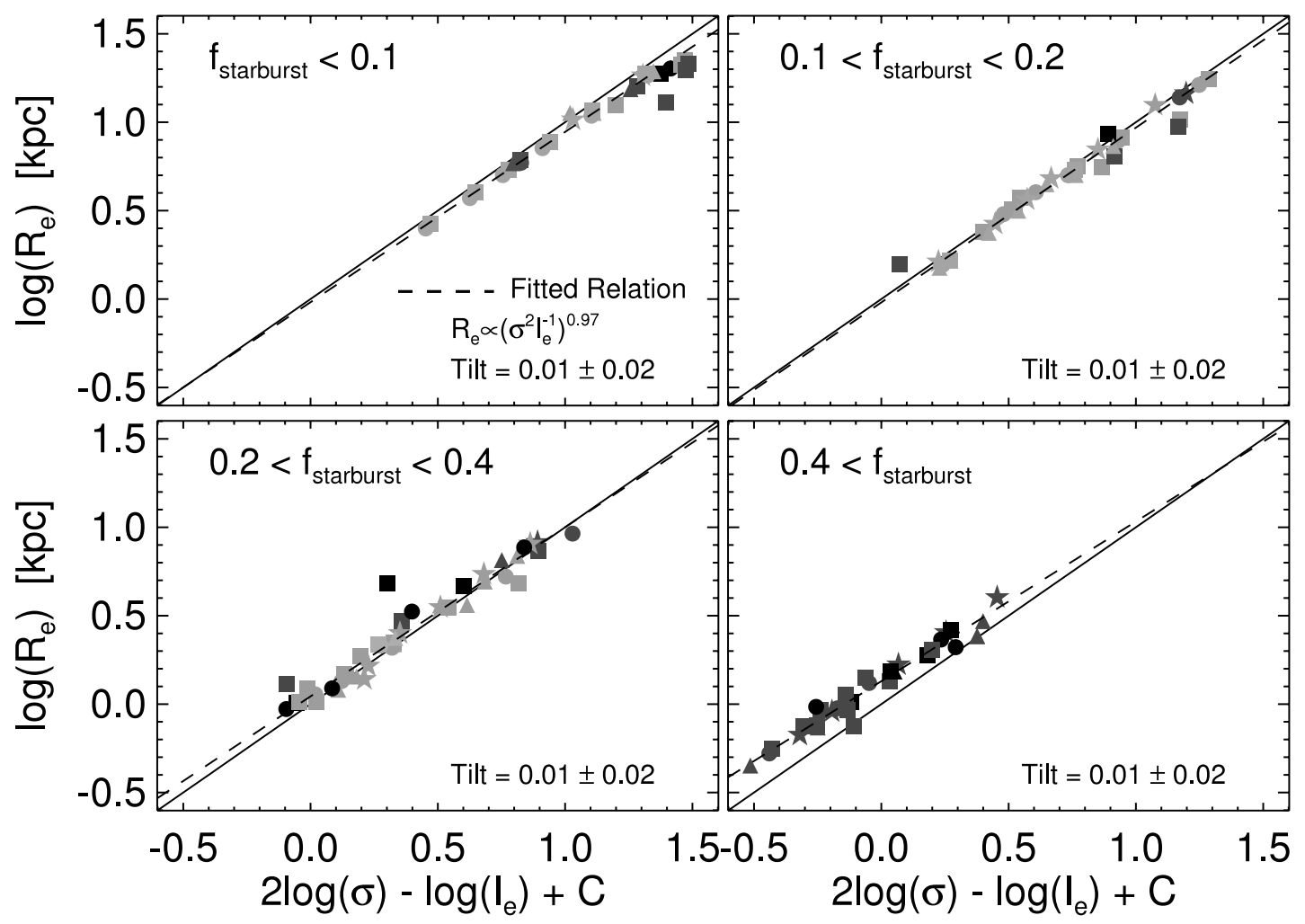

Fig. 13. - FP in narrow bins of $f_{\mathrm{sb}}$, for our simulations, as in Fig. 12, but showing an alternative projection of the FP $\left[R_{e} \propto\left(\sigma^{2} I_{e}^{-1}\right)^{1+\alpha}\right]$. The result (no tilt at fixed $\left.f_{\mathrm{sb}}\right)$ is the same, but in such a projection (with a small sample) small errors in $R_{e}$ will tend to amplify the observed tilt (and the physical meaning of the tilt is less clear). [See the electronic edition of the Journal for a color version of this figure.] 


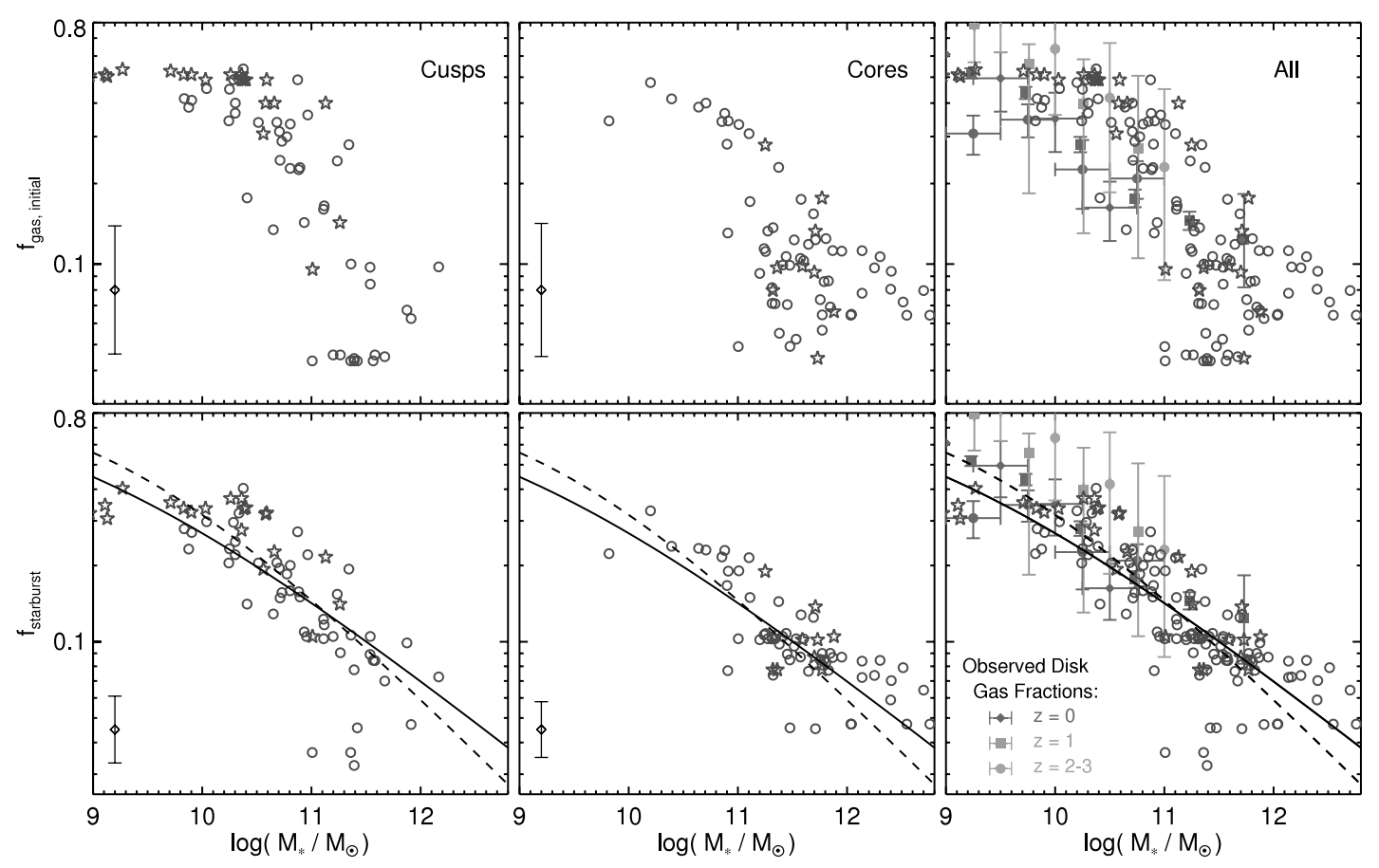

FIG. 14.- Inferred gas content (dissipational/starburst fraction) of elliptical-producing mergers as a function of stellar mass. Initial gas fraction (top) and physical final starburst mass fraction (bottom) corresponding to the best-fit simulations to each observed system in the samples of Lauer et al. (2007b) (circles) and Kormendy et al. (2008) (stars) are shown, with the typical 25\%-75\% allowed range (error bars). We show results separately for cusp ellipticals (left), core ellipticals (middle), and both together (right). The dashed (solid) line shows the fit to the data (eq. [10]) in cusp (core) ellipticals. Gray symbols with error bars indicate the mean (and $\pm 1 \sigma$ range in) disk gas fractions at the same stellar mass, at $z=0$ (Bell \& de Jong 2001; Kannappan 2004; McGaugh 2005; diamonds, squares, and circles, respectively), $z=1$ (Shapley et al. 2005; squares), and $z=2$ (Erb et al. 2006; circles). There is a clear trend of increasing dissipation required to explain elliptical profiles at lower masses (significant at $>8 \sigma$ in either core or cusp subsamples separately), in good agreement with the observed trend in progenitor disk gas fractions over the redshift range where cusp ellipticals are formed, and with what is invoked to explain the observed densities and FP correlations of ellipticals (e.g., Kormendy 1989; Hernquist et al. 1993). The best-fit trends in cusp and core populations are statistically identical; i.e., the dissipational/extra light component is preserved regardless of possible remergers. [See the electronic edition of the Journal for a color version of this figure.]

fraction $f_{\mathrm{sb}}$ on mass in cusp ellipticals (or observed gas-rich merger remnants) can be well fitted by

$$
\left\langle f_{\text {starburst }}\right\rangle \approx\left[1+\left(\frac{M_{*}}{M_{0}}\right)^{\alpha}\right]^{-1}
$$

with $\left(M_{0}, \alpha\right)=\left(10^{9.2 \pm 0.2} M_{\odot}, 0.43 \pm 0.04\right)$ (shown in Fig. 14) and a roughly constant factor of $\sim 1.5-2$ scatter at each $M_{*}$. In Paper III we demonstrated that an essentially identical scaling [a statistically equivalent $\left.\left(M_{0}, \alpha\right)=\left(10^{8.8 \pm 0.3} M_{\odot}, 0.35 \pm 0.05\right)\right]$ applies to core ellipticals, as expected if cusp ellipticals are indeed their progenitors (since identical remergers will conserve dissipational or extra light mass fractions).

We also demonstrated in Papers II and III that this empirically measured systematic dependence of dissipational mass fraction on stellar mass agrees well with the observed dependence of disk galaxy gas fractions on mass. This is exactly what is expected if ellipticals are (at least originally) formed in gas-rich, disk-disk mergers (regardless of whether or not they subsequently experience remergers). We show this in Figure 14 with the observed disk gas fractions as a function of stellar mass from Bell \& de Jong (2001), Kannappan (2004), and McGaugh (2005) at $z=0$, as well as at $z=1$ (Shapley et al. 2005) and $z=2$ (Erb et al. 2006). At all these redshifts, the trend of gas fraction is similar, and the observed gas fractions evolve relatively weakly with redshift (by a factor of $\sim 1-2$ ), bracketing the range of dissipational mass fractions observed in ellipticals at each mass.

In other words, regardless of the exact times of formation, a systematic trend similar to that observed in the dissipational fractions of ellipticals is inevitable if disks are the progenitors of ellipticals. Note that even if there is a systematic dependence of the time of first gas-rich merger on stellar mass in this range, the mixing of formation redshifts would be less important than the mean dependence of disk gas fraction on mass (if we assume that the stellar population ages date the merger times, which is an upper limit to the magnitude of this effect since the stellar ages at least in part reflect the similar trend in stellar ages of disks as a function of mass, then the systematic effects added by this age scaling are not important). The dependence of disk gas fractions on mass is therefore the primary driver of the observed dependence of dissipational mass fraction on elliptical mass.

Among other things, this implies that we could predict the dissipational mass fractions of ellipticals as a function of their mass, based on the observed disk gas fractions at each mass. We therefore pursue the following exercise: we consider the consequences of adopting the observed scaling of disk gas fractions with mass as our expectation for the dissipational fractions in ellipticals as a function of mass. Since we have just shown that they are equivalent, we obtain identical results if we select the empirically fitted dissipational fractions of ellipticals as a function of mass; in either case, the important thing is that there is a well-defined, physically motivated and observationally confirmed systematic scaling of dissipational fraction with mass.

\subsection{The Consequence for the FP}

Given the known scaling of disk gas fractions as a function of mass, we can then focus on the subsample of simulations that obey this scaling. That is, at a particular mass scale, we know the range of disk gas fractions seen empirically. We therefore consider only simulations within that range of gas fractions, as opposed to 

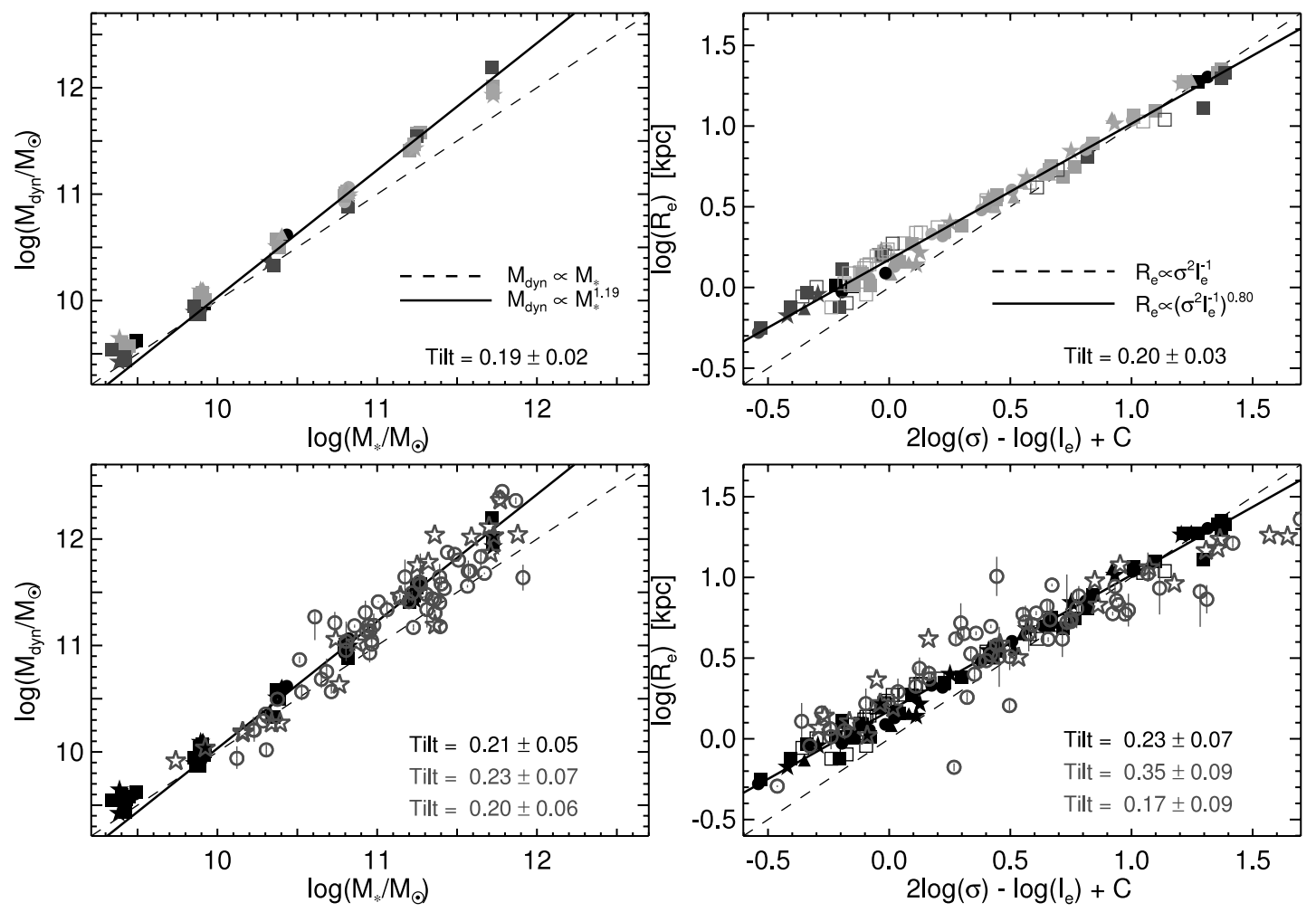

FIG. 15.-FP for all observed ellipticals (bottom), in the style of Fig. 11 (left) and Fig. 13 (right), with the measured tilt. The standard tilt $\alpha \sim 0.2$ is now apparent. We

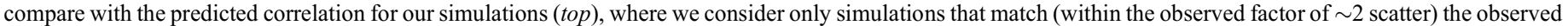

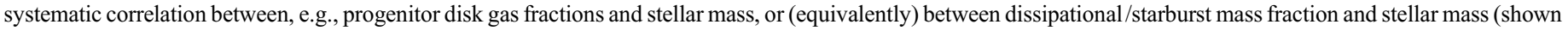

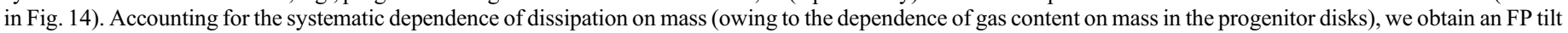
in good agreement with that observed, with comparable small scatter. [See the electronic edition of the Journal for a color version of this figure.]

others in our library that are more gas-rich or gas-poor than typical observed disks of the same stellar mass. We have considered the effects of redshift evolution (using the mean age of ellipticals as a function of their stellar mass as a proxy for their merger time, we adopt the gas fraction of disks of the appropriate mass at that redshift) and find that it makes relatively little difference (although some secondary correlations along the FP relating to stellar populations and the age of systems are discussed in $\S 11.2$ ). This restriction imposes a mean dependence of extra light or starburst mass fraction on stellar mass similar to that observed in ellipticals and anticipated from disk gas fractions. Given this constraint, we can consider the FP and other correlations obeyed by just these objects; i.e., we can ask the question, what are the scalings obeyed by systems that have the same systematic dependence of dissipational fraction on mass as expected and observed in the real universe?

Figure 15 shows the results of this exercise. Now that we account for the cosmologically expected and observed dependence of dissipational content on mass, the FP is "tilted." Fitting a power law, we obtain a value for this tilt of $\alpha=0.19 \pm 0.02$. We compare this to the observed systems, plotting the data on the same footing, since we have constructed a cosmologically representative comparison sample in our simulations. We obtain an observed tilt of $\alpha=0.21 \pm 0.05$, the canonical value in the literature. More important (since any formal fitted tilt will vary iffitted over a different dynamic range), we compare the predicted scaling directly to actual ellipticals and find that the simulations and observed systems occupy a statistically identical locus in $M_{\text {dyn }}-M_{*}$ space. As a check, we fit the Kormendy et al. (2008) and Lauer et al. (2007b) subsamples independently and obtain consistent values for the tilt.
We show the tilt again in terms of $R_{e}$ and obtain a similar result (for this projection, the formal tilt value is expected to be similar to that for $M_{\mathrm{dyn}}-M_{*}$ ). Fitting the observed samples, we find that they trace an identical locus, but the power-law fits in this case are more sensitive to dynamic range (apparent by the fact that the formal tilts from Kormendy et al. [2008] and Lauer et al. [2007b] are slightly discrepant, despite the points tracing each other where they overlap). As discussed above, caution should be used fitting in this FP projection (here the dynamic range is much larger than the fit errors in $R_{e}$, so there are not large biases introduced in such observational fits, but in a more limited subsample, results fitted in this fashion are less robust than those fitted in the more physical $M_{\text {dyn }}-M_{*}$ space, where the axes are independent). Given the larger uncertainties in this case, the agreement is reasonable. Note that the absolute values of $M_{*}$ and $M_{\text {dyn }}$ and zero points here are sensitive to our adopted virial coefficients and IMF assumptions, but the tilt and our meaningful comparisons are not.

Figure 16 compares this result with the samples divided into cusp and core ellipticals. For both classes of objects, the agreement with the simulations is good, and the two appear to trace a continuous FP correlation. This is not surprising: it has been shown that massive, boxy ellipticals with cores trace a continuous FP with less massive, disky, cuspy ellipticals (at least at the massive end, where most of the core population resides; see Gerhard et al. 2001; von der Linden et al. 2007), and we have shown that the scaling of $M_{\mathrm{dyn}} / M_{*}$ with dissipational fraction (and systematic dependence of dissipational fraction on mass) is similar in both cusp and core ellipticals (and, in simulations in Paper III, we explicitly demonstrate that this should be true for both the original gas-rich merger remnants and dry remerger remnants). Furthermore, a number of studies (Capelato et al. 1995; Dantas et al. 

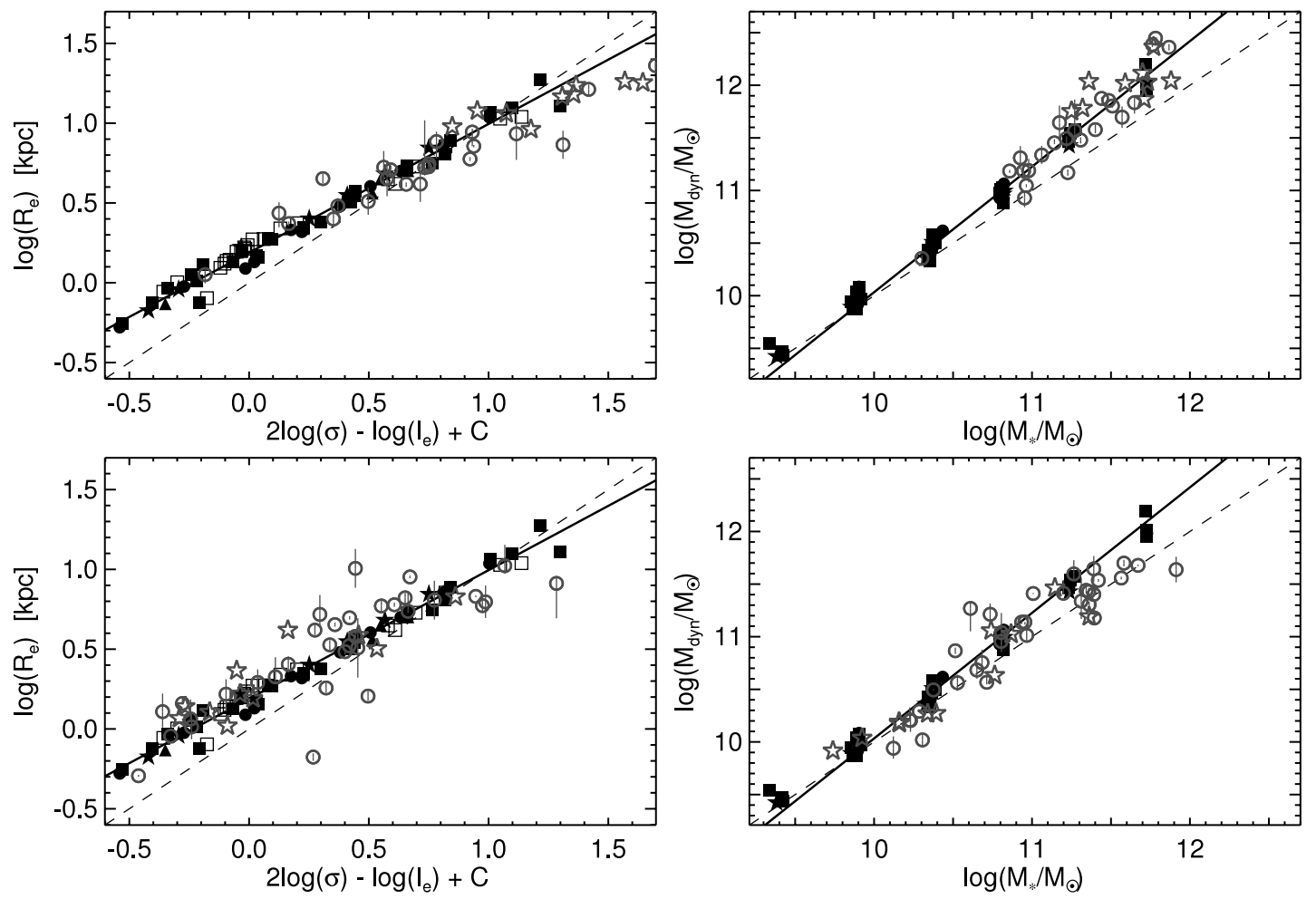

FIG. 16.-Observed FP, compared with our simulation predictions as in Fig. 15, but divided into core (top) and cusp (bottom) ellipticals. The simulation predictions agree well with the data in both cases, consistent with theoretical modeling that even a modest number of remergers do not move systems significantly off the FP correlation established by the original, gas-rich mergers. [See the electronic edition of the Journal for a color version of this figure.]

2003; Nipoti et al. 2003; Boylan-Kolchin et al. 2005; Robertson et al. 2006b) have shown that dissipationless spheroid-spheroid remergers (popular as a mechanism for producing cores in ellipticals) tend to preserve the FP, provided that the number of remergers is modest; we discuss these issues further in $\S 10$.
Given that the FP tilt in Figure 15 arises owing to dissipation, where we have shown in Figure 11 that at the same degree of dissipation the virial correlation (no tilt) is recovered, it should be possible to explicitly factor out at least some of this tilt. Figure 17 attempts this exercise: we determine the best-fit bivariate
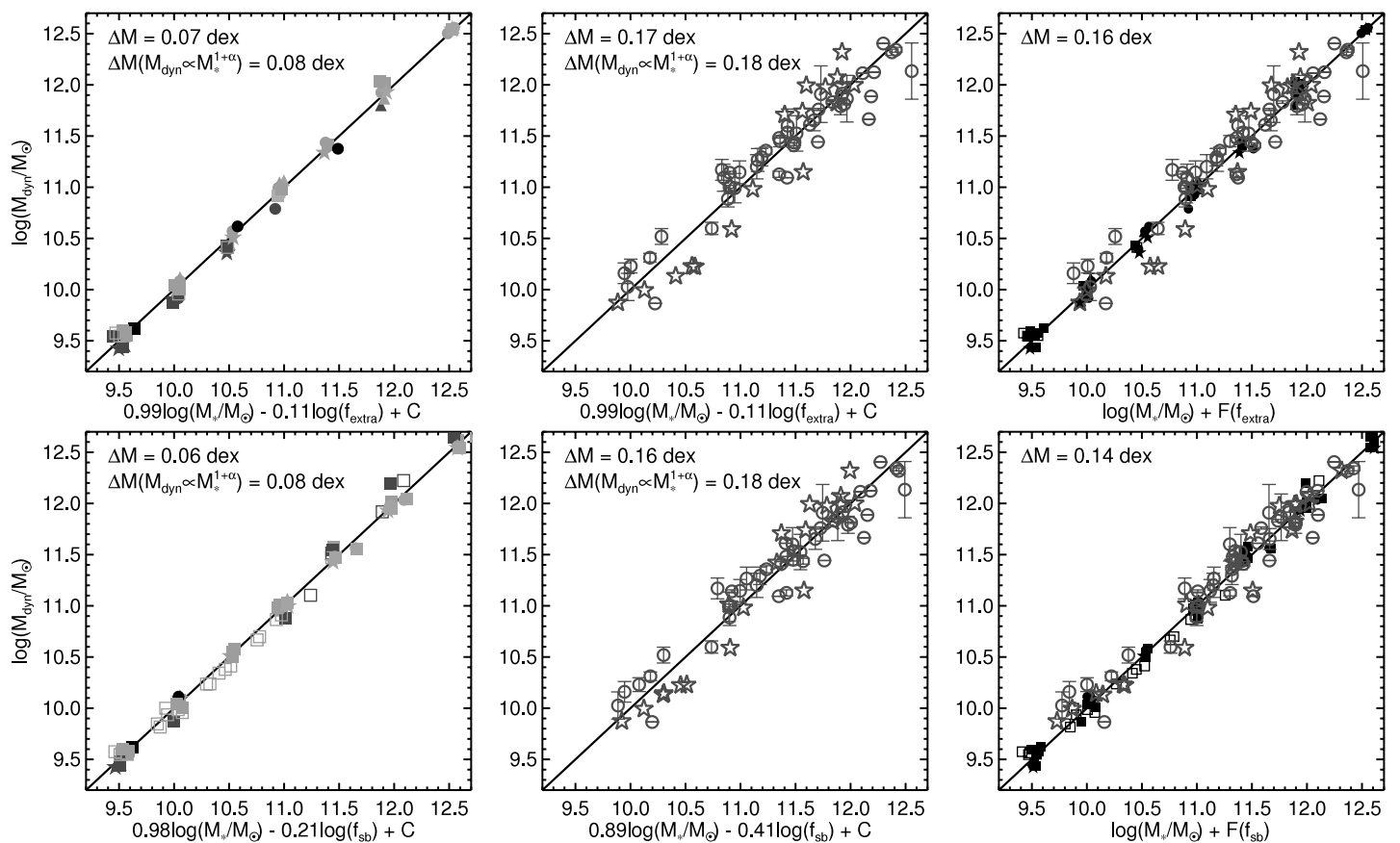

FIG. 17.- "More fundamental" representation of the FP in simulations (left; points from Fig. 15) and observations (middle and right): $M_{\mathrm{dyn}}$ as a bivariate function of $M_{*}$ and fitted extra light fraction ( $f_{\text {extra }}$, top $)$ or inferred starburst fraction ( $f_{\mathrm{sb}}$, bottom). The best-fit correlation recovers $M_{\text {dyn }} \propto M_{*}$ with the residual dependence (i.e., the tilt) owing entirely to the degree of dissipation. Compared to the FP in terms of just $M_{\text {dyn }}$ and $M_{*}$, the scatter is slightly reduced (but not much, owing to the uncertainties introduced in estimating $f_{\text {extra }}$ or $f_{\mathrm{sb}}$ ). Adopting $M_{\mathrm{dyn}} \propto M_{*}$ with the additional functional dependence of $M_{\mathrm{dyn}} / M_{*}$ on $f_{\text {extra }}$ from Fig. 7 yields a similar correlation (right) with further slightly reduced scatter. [See the electronic edition of the Journal for a color version of this figure.] 
function $M_{\text {dyn }}\left(M_{*}, f_{\text {extra }}\right)$. For simplicity and to minimize the free parameters involved, we (for now) adopt a simple power-law parameterization $M_{\text {dyn }} \propto M_{*}^{a} f_{\text {extra }}^{b}$ and determine the best-fit parameters $a$ and $b$ such that the perpendicular scatter (i.e., scatter about the least-squares bisector) is minimized; we then repeat this using the estimated starburst mass fraction $f_{\mathrm{sb}}$ instead of the fitted extra light fraction $f_{\text {extra }}$. In both cases, and regardless of whether we fit to the observations or to the simulations, the best-fit correlation is essentially the virial correlation (i.e., $a \approx 1$ ) coupled with a dependence on $f_{\text {extra }}$ or $f_{\mathrm{sb}}$. In other words, the best-fit bivariate FP assigns the FP tilt entirely to the role of dissipation: at fixed degree of dissipation, there is no tilt. Explicitly including the degree of dissipation in this manner demonstrates that it is indeed "as fundamental" as any bivariate correlation (it is preferred with respect to a purely mass-dependent $M_{\text {dyn }} / M_{*}$ ). These fitted FP projections are nearly identical to the simplest predicted FP in dissipation-driven models (also shown in Fig. 17), namely, that $M_{\text {dyn }} \propto M_{*} F\left(f_{\text {extra }}\right)$, where the function $F\left(f_{\text {extra }}\right) \propto M_{\text {dyn }} / M_{*}$ has the form seen in Figure 7 (note that this is more complex than a simple power law).

In principle, this explicit inclusion of an $f_{\text {extra }}$ or $f_{\mathrm{sb}}$ dependence should be able to account for some of the scatter in the FP and therefore might provide a plane with smaller scatter. In practice, the scatter in, e.g., $M_{\text {dyn }}\left(M_{*}, f_{\text {extra }}\right)$ is only slightly reduced relative to that in just $M_{\mathrm{dyn}}\left(M_{*}\right)$. The reasons for this are twofold. First, as discussed in $\S 8.4$, the majority of the scatter in the FP owes not to different degrees of dissipation but to the combination of sight line-to-sight line variations, dispersion in progenitor disk properties, and measurement errors. Second, as discussed in $\S 4$ and Paper II, an estimator such as $f_{\mathrm{sb}}$ or $f_{\text {extra }}$ is of course not a perfect tracer of the true starburst mass fraction, with typical factor of $\sim 2$ uncertainties. In practice, then, most of the reduction in scatter from factoring out $f_{\text {extra }}$ is negated by scatter introduced in the variation between $f_{\text {extra }}$ and the "true" starburst mass fraction. The result is that the scatter in the FP in terms of $M_{*}$ and $f_{\text {extra }}$ is comparable to or slightly less than that in terms of $M_{*}$ alone. Owing to the uncertainties in estimating $f_{\text {extra }}$, then, this FP representation is not necessarily a substantially improved observational predictor of $M_{\text {dyn }}$ or, e.g., $R_{e}$ at fixed $\sigma$ and $M_{*}$. However, the FP in these terms is certainly comparable to the traditional FP in how tightly it relates $M_{\text {dyn }}$ and $M_{*}$, and it is "more fundamental" in terms of a physical explanation for the FP tilt.

Because our primary focus in this paper is the understanding of the observed $z=0 \mathrm{FP}$ correlations, we reserve a more detailed modeling of the evolution of the physical FP (in terms of, e.g., stellar and dynamical mass) with redshift (as, e.g., typical merger histories and progenitor gas content evolve) for future work (Hopkins et al. 2008e). For now, we simply note that the evolution in physical parameters considered here is predicted to be weak, in agreement with direct observational constraints from high-redshift weak lensing (Heymans et al. 2006) and optical studies (note that there is expected evolution in optical bands owing to stellar population effects; these represent complimentary constraints on elliptical formation histories; see, e.g., di Serego Alighieri et al. 2005; Treu et al. 2005; van der Wel et al. 2005; van Dokkum \& van der Marel 2007). To the extent that these constraints agree with our expectations based on empirical estimates for the evolution of disk gas fractions, they represent independent support for the scenario outlined here.

\subsection{Projections of the FP}

As another check, we consider our results in the observed FP space of Pahre et al. (1998) in Figure 18. Here the coefficients of

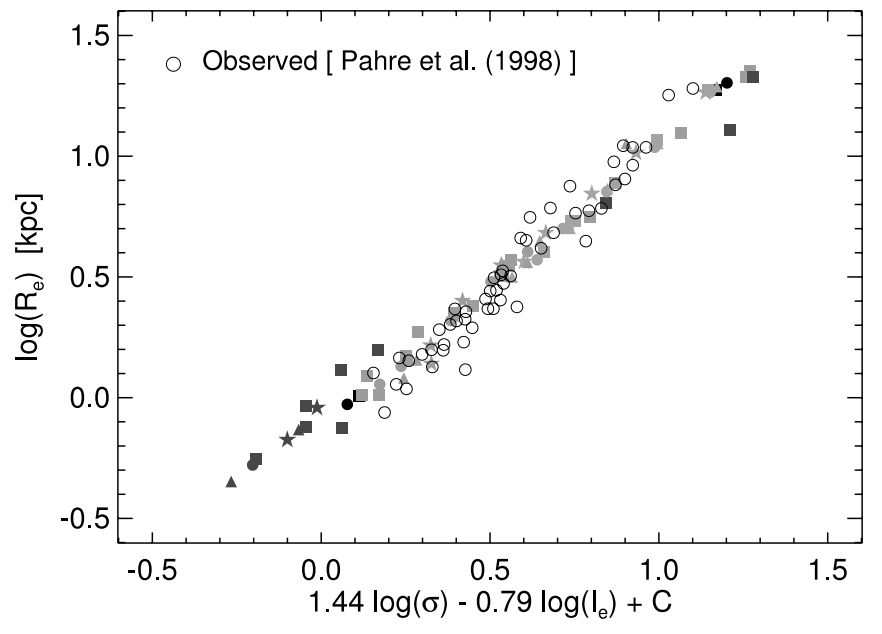

FIG. 18.-Observed FP, in the best-fit projection from Pahre et al. (1998), i.e., freeing the exponents in $R_{e} \propto \sigma^{\beta} I_{e}^{\gamma}$. Open symbols are the observed systems; filled symbols are our simulation results from Fig. 15. If the observed FP was more complex than a tilt (e.g., if $M_{*}$ depended substantially on $R_{e}$ or $\sigma$ separately at fixed $M_{\text {dyn }}$, our results would disagree here. The agreement is good, as expected based on observations that the FP is, essentially, a tilted virial plane. [See the electronic edition of the Journal for a color version of this figure.]

$\sigma, R_{e}$, and $I_{e}$ are independently free in determining the best-fit projection of the observations. If, in principle, the FP were much more complex than a simple tilt (i.e., if $M / L$ depended substantially on $R_{e}$ or $\sigma$ at fixed $M_{\text {dyn }}$ ), then the simulations might match the observations in Figures 15-21 but not in this representation. In fact, we find good agreement with the observations. This is not surprising, since it has been established that the FP can be represented as a tilt under some homology assumptions (e.g., Jorgensen et al. 1996; Padmanabhan et al. 2004).

Figure 19 plots projections of the FP, specifically the size-mass relation and the velocity dispersion-mass (Faber-Jackson) relation. We show these correlations for the same simulations used to construct the FP in Figures 15-18; since we showed in $\S 6.1$ that, e.g., effective radii scale systematically with dissipational fraction, a robust prediction of the size-mass relation requires that the simulations considered have appropriate dissipational fractions for systems of their stellar mass. We show sizes and velocity dispersions as a function of stellar mass because this is how these correlations are generally presented in the literature, but differences are small and the comparison with observations is similar if we consider them as a function of dynamical mass.

We compare with the same correlations in the observed samples for which we consider the FP and with the mean correlations measured for ellipticals in the SDSS. The simulations agree well with the observations, both those that we consider in our FP analysis and the trends in the general population (it is also reassuring that our observed FP samples obey the same projected scalings, with similar scatter). The scatter in these correlations is larger than that between $M_{\text {dyn }}$ and $M_{*}$, since at fixed $M_{\text {dyn }}$ there is a trade-off between $R_{e}$ and $\sigma$. It is also interesting to note that the trends show significant curvature: their combination $\left(M_{\mathrm{dyn}}-M_{*}\right)$ is reasonably approximated by a power-law scaling, but either the $R_{e}-M_{*}$ or $\sigma-M_{*}$ slope will vary with the fitted mass interval (for this reason, we refrain from quantifying a power-law fit to either the simulations or observations, but we note that if we do so over the same mass interval for both, we obtain the same result).

This has been discussed in the literature, for both the $R_{e}-M_{*}$ and $\sigma-M_{*}$ relations (see, e.g., Lauer et al. 2007a; von der Linden et al. 2007; Desroches et al. 2007; note that even Shen et al. [2003] 

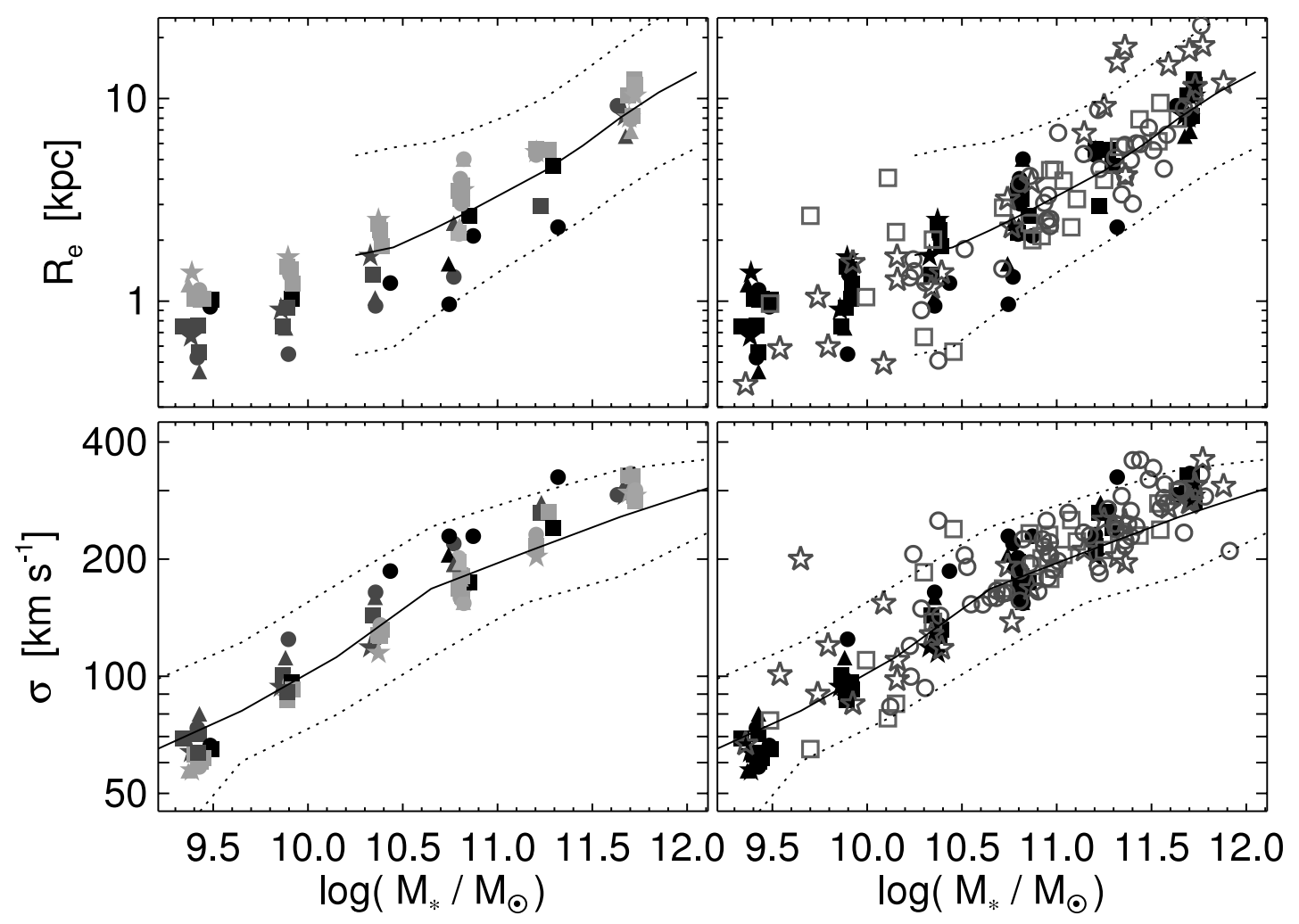

FIG. 19.- Projections of the FP. We show the size-mass (top) and velocity dispersion-mass (Faber-Jackson; bottom) correlations, for the same subset of simulations used in our FP predictions in Fig. 15 (left) and observations (right). The observed mean relations for SDSS ellipticals (solid lines; with $295 \%$ range as dashed lines) are shown over the range of data used in the observed fits (Shen et al. [2003] and Gallazzi et al. [2006] for size-mass and velocity dispersion-mass relations, respectively). Note the curvature in both relations, owing to the dependence of dissipation on mass and its role in setting both $R_{e}$ and $\sigma$. The simulations and observations agree with each other and with the mean correlations observed in much larger samples; i.e., once systems with the appropriate range of dissipational fraction for their mass are selected, they follow the observed size-mass and other correlations. The relations would not agree if we used systems with significantly different dissipational fractions as a function of mass. [See the electronic edition of the Journal for a color version of this figure.]

see tentative evidence for this effect in their lowest mass bins, but their dynamic range at the low-mass end is limited). We note here that such curvature can arise even from pure gas-rich merger remnants, owing to the dependence of the amount of dissipation on mass and corresponding scalings of size and velocity dispersion. This is supported by the fact that both our cusp and core subsamples independently exhibit similar curvature. It has also been observed that non-BCG and BCG galaxy samples show similar curvature (despite the latter being much more likely to have experienced dry mergers; see, e.g., de Lucia \& Blaizot 2007). This is not to say that remergers will not introduce curvature in these relations or move systems considerably with respect to them (see, e.g., Boylan-Kolchin et al. 2006), but rather to emphasize that dry remergers are not the only source of curvature in the relations, and curvature should not necessarily be interpreted as evidence for dry mergers (further note that systems cannot move too far off their initial correlations, as they are constrained by the observed scatter).

It is well established that these correlations are different from those obeyed by spiral galaxies. If we do crudely approximate the correlations as power laws (for illustrative purposes) over the dynamic range shown in Figure 19, we obtain correlations $R_{e} \propto$ $M_{*}^{0.56}$ (as in Shen et al. 2003) and $\sigma \propto M_{*}^{0.25}$ (see, e.g., Bernardi et al. 2003), also yielding $R_{e} \propto \sigma^{2.0}-\sigma^{2.2}$. Compare these to the observed relations in disks: $R_{e} \propto M_{*}^{0.30}-M_{*}^{0.35}, V_{\max } \propto M_{*}^{0.27}$ $M_{*}^{0.30}$, and $R_{e} \propto V_{\max }^{1.0}-V_{\max }^{1.2}$ (see, e.g., Persic et al. $1996 \mathrm{~b}$; Courteau et al. 2007; Avila-Reese et al. 2008). These differences relate to the tilt of the FP and the fundamental distinctions at issue here: at high masses, ellipticals and disks have similar sizes, but at low masses, ellipticals are much smaller. The difference, in our simulations, arises because of changes in the degree of dissipation, reflected in the extra light content. In principle then, it should be possible to consider the resulting correlations between $R_{e}$ and $M_{*}$ or $\sigma$ in terms of the combination of their dependence on that variable and the additional dependence on $f_{\text {extra }}$ or $f_{\mathrm{sb}}$.

Figure 20 shows the results of this exercise: we consider $R_{e}$ as a bivariate function of combinations of $M_{*}, \sigma$, and $f_{\text {extra. }}$. The correlation $R_{e}\left(M_{*}, \sigma\right)$ represents the traditional FP correlation: that the correlation is not simply $R_{e} \propto M_{*} / \sigma^{2}$ reflects the tilt of the FP. If instead we fit $R_{e}$ to a combination of $M_{*}$ and $f_{\text {extra }}$, we obtain a fit of similar quality (i.e., this combination is an equivalent predictor of $R_{e}$ ), but with the dependence $R_{e} \propto M_{*}^{0.3} f_{\text {extra }}^{-0.57}$. This is an equivalent FP in terms of its usefulness as a predictor of $R_{e}$, with a scatter $\sim 0.15-0.20$ dex. More interesting, the explicit dependence of $R_{e}$ on $M_{*}$ fitted in this manner is identical to that observed in disks $\left(R_{e} \propto M_{*}^{0.3}\right)$, with the remaining dependence owing to the dependence of $R_{e}$ on $f_{\text {extra }}$. Given the cosmological scaling of $f_{\text {extra }}$ with $M_{*}$, we obtain the steeper $R_{e} \propto M_{*}^{0.6}$ relation for ellipticals when $f_{\text {extra }}$ is ignored, but at fixed $f_{\text {extra }}$ or $f_{\text {sb }}$ (i.e., fixed degree of dissipation) a relation $R_{e} \propto M_{*}^{0.3}$, similar to that observed in disk galaxies, is observed (in line with the predictions from Robertson et al. 2006b). We obtain the same results for the size-velocity dispersion relation: a best-fit $R_{e} \propto \sigma^{1.33} f_{\text {extra }}^{-0.39}$ (or $R_{e} \propto \sigma^{0.94} f_{\text {extra }}^{-0.89}$ ); the dependence of $R_{e}$ on $\sigma$ at fixed $f_{\text {extra }}$ or $f_{\mathrm{sb}}$ is very similar to the dependence of $R_{e}$ on rotational velocity $V_{\max }$ in disks (to lowest order, our simulations and other numerical experiments demonstrate that $\sigma$ traces the premerger $V_{\max }$, modulo a roughly constant normalization offset owing to, e.g., 

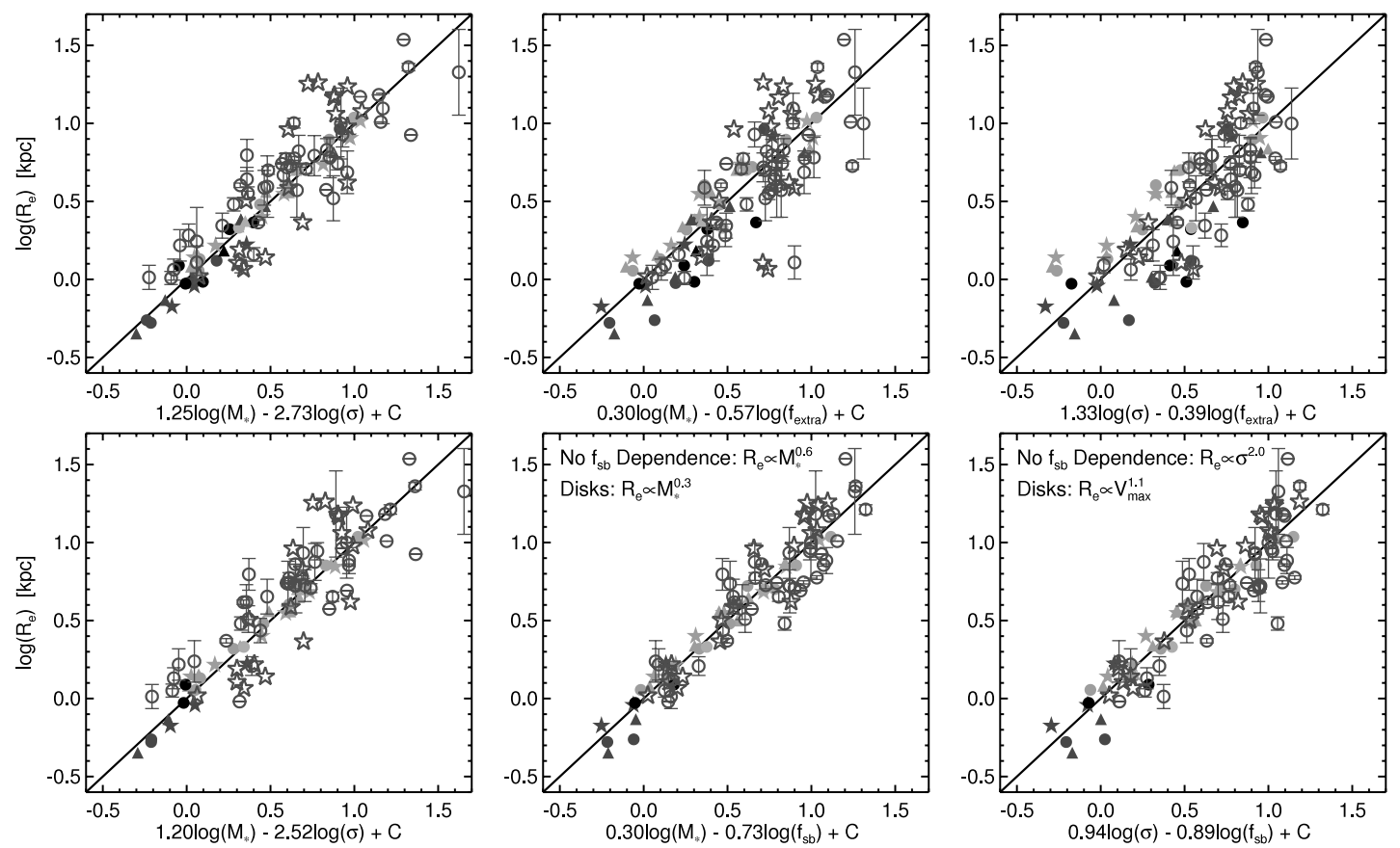

FIG. 20.-FP predictors of $R_{e}$. Left: $R_{e}$ as a function of the traditional global parameters $M_{*}$ (stellar mass) and $\sigma$ (velocity dispersion). Simulations (the same as used for the predicted FP in Fig. 15) and observations are shown (as in Fig. 7). The scatter in $R_{e}$ in this projection is $\sim 0.2$ dex. Middle: $R_{e}$ as a bivariate function of $M_{*}$ and fitted extra light fraction ( $f_{\text {extra }}$; top $)$ or inferred starburst fraction $\left(f_{\mathrm{sb}} ;\right.$ bottom). This is an equivalent or better observational predictor of $R_{e}$ (scatter $\sim 0.15$ dex). Right: $R_{e}$ as a function of $\sigma$ and $f_{\text {extra }}(t o p)$ or $f_{\mathrm{sb}}\left(\right.$ bottom). Again, this is an equivalent predictor (scatter $\sim 0.2$ dex). Ignoring the effects of dissipation, we recover the $R_{e}-M_{*}$ and $\sigma-M_{*}$ relations in Fig. 19, with very different slopes from the relations for disks (comparison shown here). However, with the effects of dissipation explicitly included here, the relation between $R_{e}$ and $M_{*}$ or $R_{e}$ and $\sigma$ is equivalent to that obeyed by spirals; i.e., the difference between the "projected" elliptical and spiral scaling relations is entirely attributable to the degree of dissipation $\left(f_{\mathrm{extra}}\right.$ or $\left.f_{\mathrm{sb}}\right)$. [See the electronic edition of the Journal for a color version of this figure.]

the profile shape and kinematics). In other words, the difference between the observed size-mass-velocity dispersion relations in disks and ellipticals can be entirely accounted for by dissipation.

\subsection{The Small Scatter in the FP}

A final requirement for any model of the FP is that it account for the small observed scatter. Analyzing the relations predicted by the simulations, which broadly sample orbital parameters and stellar masses and fully cover the observed range of dissipational fractions at each mass, we can see directly in Figures 15-21 that there is small scatter. Formally, we find a nearly symmetric $1 \sigma$ scatter of $\sim 0.07-0.08$ dex in $M_{\text {dyn }}\left(M_{*}\right)$ or $\sim 0.065-0.075$ dex in $M_{*}\left(M_{\mathrm{dyn}}\right)[0.055$ dex scatter perpendicular to the best-fit correlation, or equivalently in the $R_{e}$ projection of the FP, depending weakly on how we define our expected $f_{\text {extra }}\left(M_{*}\right)$ and whether we use $M_{\text {true }}$ or $\left.M_{\text {dyn }}\right]$.

This is the scatter obtained in these correlations using the median values of $M_{*}$ and $M_{\mathrm{dyn}}$ for each galaxy (across $\sim 100$ sight lines): if we include the sight line-to-sight line variance in the simulations, we obtain $\sim 0.14$ dex total scatter in $M_{\text {dyn }}\left(M_{*}\right)$ ( 0.09 dex scatter perpendicular to the correlation). This is still less than that observed in each case: for the observed samples herein, we find $\sim 0.18$ dex scatter in $M_{\mathrm{dyn}}\left(M_{*}\right)$ and $\sim 0.15$ dex scatter in $M_{*}\left(M_{\text {dyn }}\right)$, i.e., a perpendicular scatter of $0.12 \mathrm{dex}$, with additional observational errors in $R_{e}$ and $M_{*}$ (estimated from our experiments in Paper II) likely contributing most of the difference (for typical $\sim 0.1$ dex stellar population model errors in $M_{*}$, this yields roughly the observed scatter).

The origin of the small intrinsic scatter in the FP is straightforward to understand in this scenario. Some intrinsic variation will come from the scatter in the total baryon-to-dark matter content of the progenitor galaxies. However, observations of the baryonic Tully-Fisher relation suggest that the scatter in $M_{*}$ at fixed maximum circular velocity (a proxy for halo mass) is small, $\approx 0.1$ dex (at least over the mass range of interest here; Bell \& de Jong 2001). Even considering the total baryon-to-halo mass ratios, which extend well beyond $R_{e}$ and are therefore of less interest, observations imply quite small scatter $\sim 0.15 \mathrm{dex}$ (Wang et al. 2006; Conroy et al. 2006; Weinmann et al. 2006). This effectively subsumes a number of quantities, including, e.g., scatter in the initial radii of the disks at fixed mass, in halo concentrations, and in other parameters (note that the scatter in any one of these quantities is not important, given that the final Tully-Fisher relation scatter is small). The contribution of this initial scatter will actually be reduced after a gas-rich merger because the quantity of interest is the ratio $\left[M_{*} / 2+M_{\mathrm{dm}}\left(<R_{e}\right)\right] /\left(M_{*} / 2\right)$. Since the merger channels gas into the center and raises the central density, the final effective radius is smaller (explaining the smaller sizes of ellipticals relative to spirals at low mass). Thus, while $M_{*}$ is just the baryonic mass of the progenitor, the contraction of the remnant makes the relative value of $M_{\mathrm{dm}}\left(<R_{e}\right)$ smaller, reducing the importance of initial scatter in $M_{\mathrm{dm}} / M_{\text {baryon }}$ to the final FP scatter. Analysis of the simulations suggests that a realistic initial $\sim 0.1$ dex scatter in the baryonic Tully-Fisher relation contributes $\lesssim 0.04-0.05$ dex scatter directly to the final FP prediction.

However, there will be additional intrinsic scatter contributed by different degrees of dissipation in systems of the same mass. Figures 6 and 8 show that at fixed stellar mass, systems with different degrees of dissipation have correspondingly different effective radii and ratios $M_{\mathrm{dyn}} / M_{*}$. However, the scatter in the expected (and observationally inferred) dissipational fraction at each mass is small, a factor $\leqslant 2$, and the dependence of $M_{\mathrm{dyn}} / M_{*}$ (see Fig. 7) on dissipational fraction over this relatively narrow range is not strong. Combining this scatter with the dependence of $M_{\mathrm{dyn}} / M_{*}$, we expect it to contribute about $\sim 0.06-0.08$ dex of intrinsic scatter. Together, this yields the relatively small $\sim 0.08$ dex intrinsic 

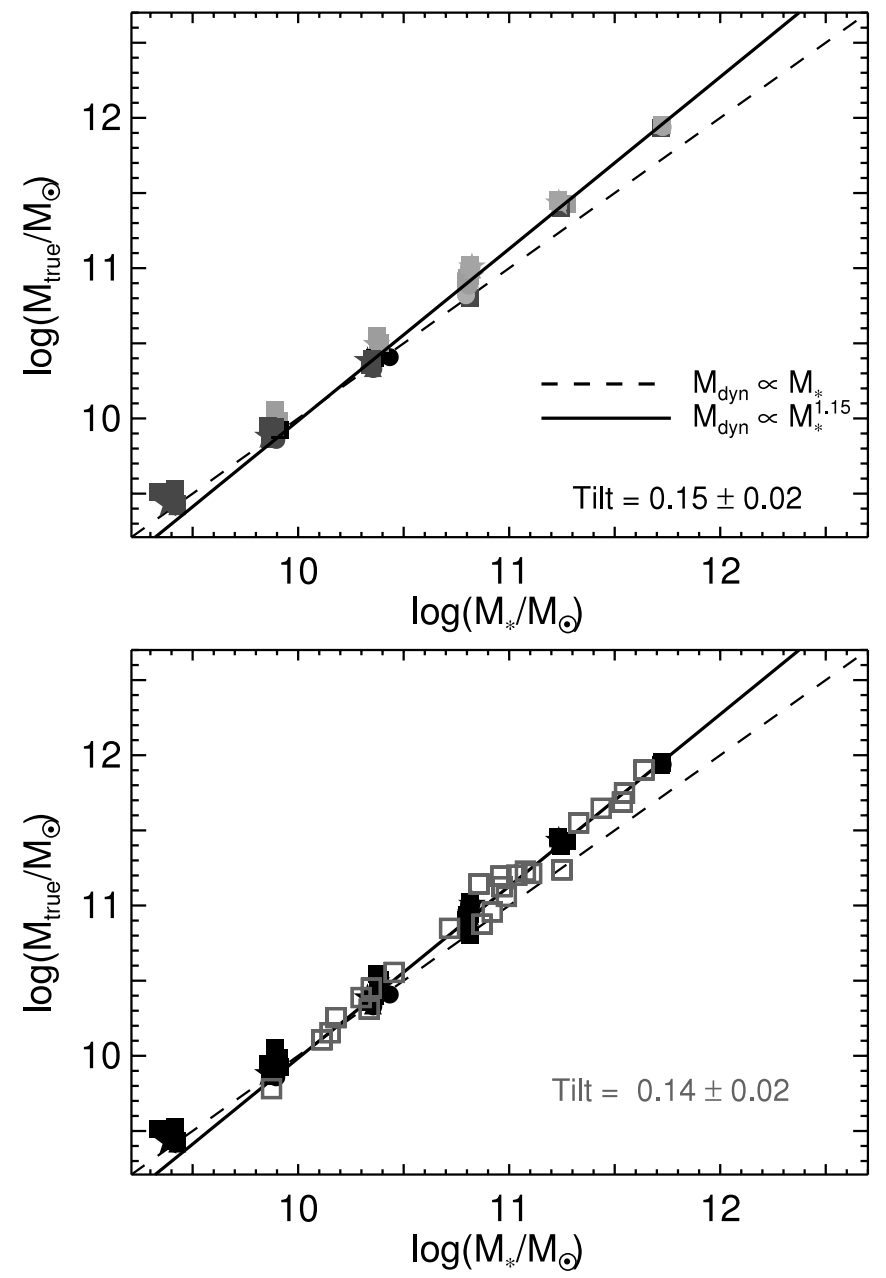

FIG. 21.- Predicted and observed FP (as in Fig. 15), but using the true total enclosed mass $M_{\text {true }}$ (stellar plus dark matter) within $R_{e}$ instead of $M_{\text {dyn }}$. We compare with the observed systems from Cappellari et al. (2006), who use integral Jeans/Schwarzschild modeling to determine a "true" mass independent of a homology or constant $M / L$ assumption. A nearly identical tilt is predicted and observed; i.e., the predicted and observed FP is really a reflection of the tilt in $M_{\text {true }} / M_{*}$ within $R_{e}$, not a tilt in $M_{\text {true }} / M_{\mathrm{dyn}}$. [See the electronic edition of the Journal for a color version of this figure.]

scatter in $M_{\mathrm{dyn}}\left(M_{*}\right)$ (before sight line-to-sight line and measurement errors).

\section{TOTAL VERSUS DYNAMICAL MASS: INVARIANCE OF THE FP}

In Figure 21 we repeat our experiment from Figure 15; i.e., we construct the FP from the simulations, given the observed and expected dependence of dissipational fraction on mass. However, instead of plotting the traditional dynamical mass estimator $M_{\text {dyn }} \propto \sigma^{2} R_{e}$, we plot the true total projected mass (stellar plus dark matter plus gas, although gas is generally negligible) within $R_{e}$, which we extract directly from the simulations and refer to as $M_{\text {true }}$. The predicted FP is nearly identical to that predicted using the $M_{\mathrm{dyn}}$ estimator; i.e., the FP predicted does, in fact, arise from a change of the dark matter-to-luminous matter ratio within $R_{e}$, owing to dissipation contracting the stellar effective radius (i.e., a change in $M_{\text {true }} / M_{*}$ with mass), rather than to traditional structural or kinematic nonhomology (which would imply a constant ratio of $M_{\text {true }} / M_{*}$, with a changing apparent mass from the dynamical mass estimator, i.e., a varying $M_{\text {true }} / M_{\text {dyn }}$ with mass).
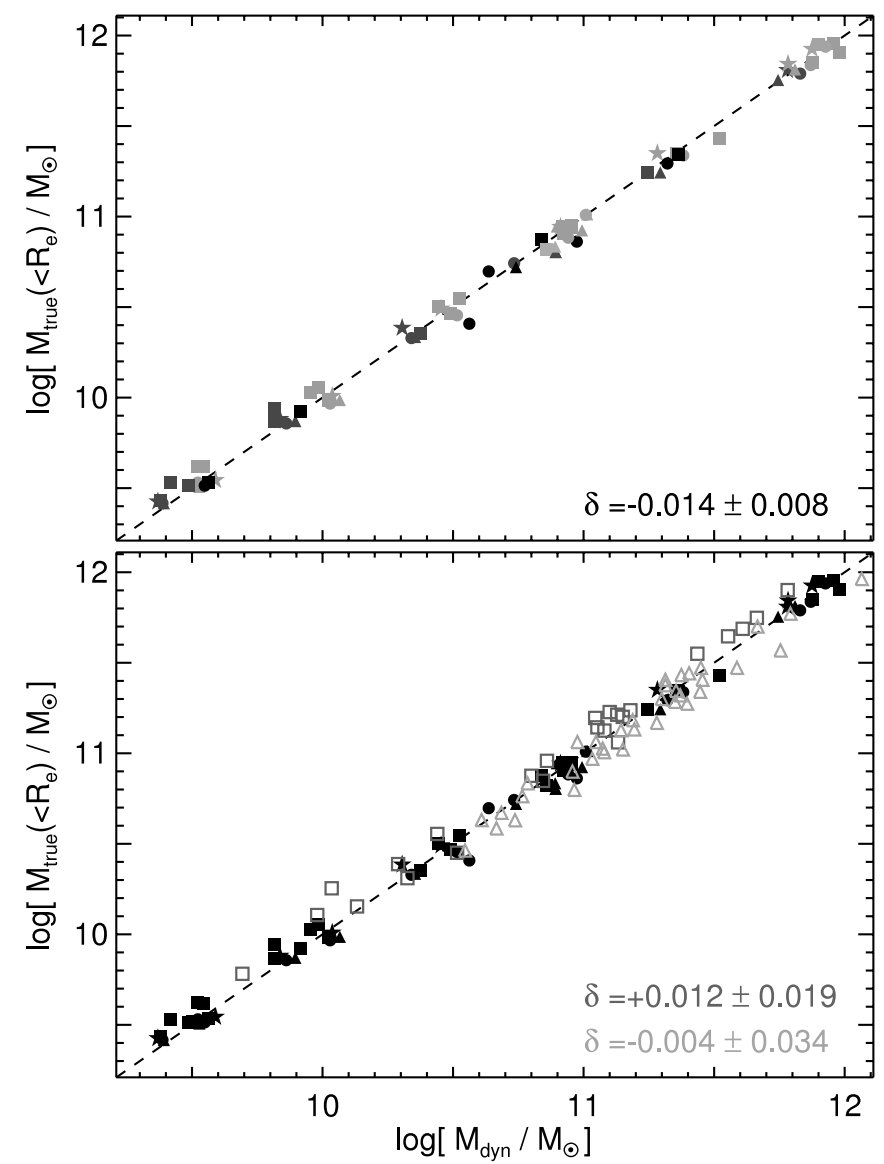

FIG. 22.-Top: Correlation between the dynamical mass estimator $M_{\text {dyn }}=$ $3.8 \sigma^{2} R_{e} / G$ and the true total mass $M_{\text {true }}$ (stellar plus dark matter) enclosed within $R_{e}$ (as in Fig. 21), for the same simulations shown in our predicted FP (Fig. 15). The dashed line is a fit of the form $M_{\text {true }} \propto M_{\mathrm{dyn}}^{1+\delta}$, with the best-fit value of $\delta$ shown: the best fit is indistinguishable from the $M_{\text {true }}=M_{\text {dyn }}$ line (i.e., has no tilt). Bottom: Same as the top panel, but for the observed systems from Fig. 21, using integral modeling (Cappellari et al. 2006; squares) or lensing mass maps (Bolton et al. 2007, 2008; triangles) to estimate the true mass $M_{\text {true. }}$. The best-fit coefficients $M_{\text {true }} \propto M_{\mathrm{dyn}}^{1+\delta}$ are shown for each observed sample. The simulations we use to predict the FP, as well as the observed systems on the FP, trace a nearly identical $M_{\text {true }} \propto \sigma^{2} R_{e}$ relation consistent with the traditional observational definition of the homology assumption. [See the electronic edition of the Journal for a color version of this figure.]

This is despite the systematic variation of extra light fraction with mass (reflecting the systematic dependence of dissipational or initial disk gas fraction). In other words, while there is technically a subtle nonhomology implicit in the fact that the dissipational fraction depends on mass (and, as a result, the fitted $f_{\text {extra }}$ and $f_{\mathrm{sb}}$ change with mass, whereas they would be constant for true perfectly self-similar systems), it does not contribute any significant or observationally meaningful structural or kinematic nonhomology. The "homology assumption," namely, that the ratio of the dynamical mass estimator to the true enclosed mass within the effective radius $\left(M_{\text {true }} / M_{\text {dyn }}\right)$ is constant, is predicted to hold, while the FP is satisfied.

Figure 22 demonstrates this explicitly: we plot the ratio of true enclosed mass to the dynamical mass estimator, specifically for the same simulations we used to predict the FP and its tilt. The ratio $M_{\text {true }} / M_{\text {dyn }}$ is constant despite changing dissipational fractions; fitting a power law $M_{\text {true }} \propto M_{\text {dyn }}^{1+\delta}$, we obtain $\delta=-0.01 \pm$ 0.01 (both insignificant and completely negligible compared to the observed value of the FP tilt, $\alpha \approx 0.2$ ); i.e., the homology assumption is valid in the observational sense as applied to these simulations, and there is no significant implied systematic structural 
or kinematic nonhomology (in the traditional sense of the term) as a function of mass.

We compare these predictions with observed "true" enclosed masses within the effective radii of observed systems, using two different approaches for estimating the true enclosed mass (without explicitly invoking the homology assumption). First, Cappellari et al. (2006) estimated total masses from three-dimensional Schwarzschild modeling or two-dimensional Jeans modeling of local ellipticals with two-dimensional velocity field information from SAURON maps (see also Emsellem et al. 2007; McDermid et al. 2006; Cappellari et al. 2007) and used stellar population models to estimate stellar masses (we correct these for the choice of IMF but otherwise do not modify them). We also have considered integral modeling masses from other, independent sources (e.g., van der Marel 1991; Kronawitter et al. 2000; Häring \& Rix 2004) and obtain identical results in each case.

Figure 21 plots the FP of these objects, i.e., the true enclosed mass determined in this manner as a function of stellar mass. The tilt, in agreement with the simulations, is nearly identical to that given by the dynamical mass estimator $M_{\text {dyn }}$. This is clear in Figure 22, where we compare the predicted correlation between $M_{\text {dyn }}$ and $M_{\text {true }}$ with these observations (we apply the same definition of $M_{\text {dyn }}$ to the observed objects as to the simulations, namely, $\left.M_{\text {dyn }}=3.8 R_{e} \sigma^{2} / G\right)$. As noted by Cappellari et al. (2006), there is a tight correlation between $M_{\text {dyn }}$ and $M_{\text {true }}$ without any significant deviation in this space from the homology assumption $(\delta=$ $+0.012 \pm 0.019)$. Not only does this tight proportionality $(\delta \approx 0)$ agree with our simulations, but the normalization fitted to the observed sample, i.e., mean ratio $M_{\text {true }} \approx 3.8 R_{e} \sigma^{2} / G$, agrees with that predicted by the simulations over the observed mass range to better than $0.03 \mathrm{dex}$, further suggesting that the structural properties and profile shapes of the simulations are in good agreement with those observed.

Second, Bolton et al. $(2007,2008)$ have performed a similar exercise, but using instead the enclosed masses determined from gravitational lensing. We compare their FP [i.e., $\left.M_{\text {true }}\left(M_{*}\right)\right]$ in Figure 21 and again find similar tilt to that predicted by the simulations (using the true enclosed mass within $R_{e}$ as $M_{\text {true }}$ ) and to that obtained using $M_{\text {dyn }}$ instead of $M_{\text {true }}$. Figure 22 shows that, for these objects as well, $M_{\text {true }} \propto M_{\text {dyn }}(\delta=-0.004 \pm 0.034 \approx 0)$, with again a normalization (mean $M_{\text {true }} / M_{\text {dyn }}$ ) in agreement with the simulations to within 0.02 dex. Note that the authors restricted themselves to true masses and dynamical masses within $R_{e} / 2$; if we use their lens models to correct the true masses to $R_{e}$ and take the corresponding dynamical masses at that radius, we obtain identical results.

The simulations reproduce the observed, nearly exact proportionality between $M_{\text {true }}$ and $M_{\text {dyn }}$ and therefore show the same FP behavior as has been seen in the observations, namely, that the FP tilt remains similar regardless of whether $M_{\text {dyn }}$ or $M_{\text {true }}$ is considered. This demonstrates the point from $\S 5$, that nonhomology (in the general, observationally motivated sense of different $M_{\text {true }} / M_{\text {dyn }}$ as a function of galaxy properties) is not a significant contributor to the FP tilt.

\section{REMERGERS AND FP EVOLUTION}

The predictions we seek to test, as well as the observed dependence of FP tilt on the degree of dissipation that we have demonstrated, hold in the same manner for both cusp and core ellipticals. However, a number of observed properties (see, e.g., Faber et al. 1997) suggest that core ellipticals may be the products of subsequent dry or spheroid-spheroid, relatively gas-free remergers of (generally lower mass) cusp ellipticals (formed directly in gasrich mergers). If true, do we expect the level of agreement we see?
The answer is generally yes, provided that the number of remergers is not large. Numerical experiments (e.g., Capelato et al. 1995; Dantas et al. 2003; Nipoti et al. 2003; Boylan-Kolchin et al. 2005; Robertson et al. 2006b) indicate that remnants of dissipationless mergers of systems that begin on the FP tend to remain on the FP. Even if the remerger introduces no new tilt, its effects would be small. To lowest order, since the gas is mostly exhausted, the remerger will be dissipationless, and merging two identical systems $M_{1}$ and $M_{2}$ on a parabolic orbit, one expects their profiles to be roughly preserved. This leads to the energy conservation equation

$$
E_{f}=k\left(M_{1}+M_{2}\right) \sigma_{f}^{2}=E_{i}=k M_{1} \sigma_{1}^{2}+k M_{2} \sigma_{2}^{2},
$$

where $\sigma_{f}$ is the velocity dispersion of the final remnant and $k$ is a constant that depends on the shape of the profile. From this, one obtains the general rule that a major 1:1 merger will approximately double $R_{e}$ while preserving $\sigma$, doubling both $M_{\mathrm{dyn}}$ and $M_{*}$ (e.g., Hausman \& Ostriker 1978; Hernquist et al. 1993).

Relative to the FP scaling, $M_{\text {dyn }} \propto M_{*}^{1.2}$, the remnant is now $\sim 0.06$ dex below the FP expectation, only $\sim 0.3-0.5 \sigma$ ( $\sigma$ being the observed FP scatter). So even if the system increases its mass by a total factor of $\sim 4-5$ via dry remergers (i.e., has $\sim 2$ equalmass remergers or $\sim 5-6$ more typical 1:3 mass ratio remergers), it will move by only $\sim 1 \sigma$ with respect to the FP.

Compare this to the impact of dissipation, which, as we demonstrate in $\S 6$, can change the effective radius and ratio $M_{\text {dyn }} / M_{*}$ (at fixed stellar mass) by nearly an order of magnitude. We therefore expect that, unless a system has experienced an extreme number of major dry remergers (expected only for the rarest, most massive central cluster galaxies), the degree of dissipation (i.e., properties we study herein) should be the dominant factor determining the effective radii, ratio of dynamical to stellar mass, and location with respect to the FP.

Furthermore, it has been noted (Boylan-Kolchin et al. 2005, 2006; Ciotti et al. 2007) that remergers can slightly increase $M_{\text {dyn }} / M_{*}$, essentially causing systems to move nearly parallel to the FP. We consider a sample of remerger simulations, described in $\S 2$, in order to demonstrate this in Figure 23. Specifically, these are identical (mass ratio 1:1) remergers of remnants of previous gas-rich, disk-disk mergers, merged in various orbital configurations. The details are discussed in $\S 2$, but we note that although we plot only identical remergers here (for illustrative purposes), the results scale appropriately for different mass ratios and various mixed encounters (i.e., merging different spheroid remnants of similar mass). Plotting both the pre- and post-remerger systems on the FP in Figure 23, we see that they move nearly parallel to the fitted relation $(\alpha \approx 0.2)$. Quantitatively, the median increase in dynamical-to-stellar mass ratio is $\sim 0.06 \mathrm{dex}$, precisely as needed to move the system along the FP (to offset the $\sim 0.06$ dex offset from the FP estimated above, if $M_{\mathrm{dyn}} / M_{*}$ were exactly conserved in a remerger).

This effect is subtly related to, e.g., changes in the orbital isotropy and kinematics of the remnant in a remerger but primarily owes to a real (albeit small) physical increase in the ratio of enclosed dark matter mass within the stellar effective radius (the progenitors and remnants obey the homology assumption as in Fig. 22, and we see an almost identical effect plotting the true enclosed mass instead of $\left.M_{\text {dyn }}\right)$. Considering the initial and final distributions of stellar and dark matter particles as a function of their initial radius, it is straightforward to understand this effect.

Despite the nearly uniform inflation of the light profile in a remerger, there is substantial scattering of stars (and dark matter); i.e., although stars at some initial $r_{i}$ will be, at the end of the 

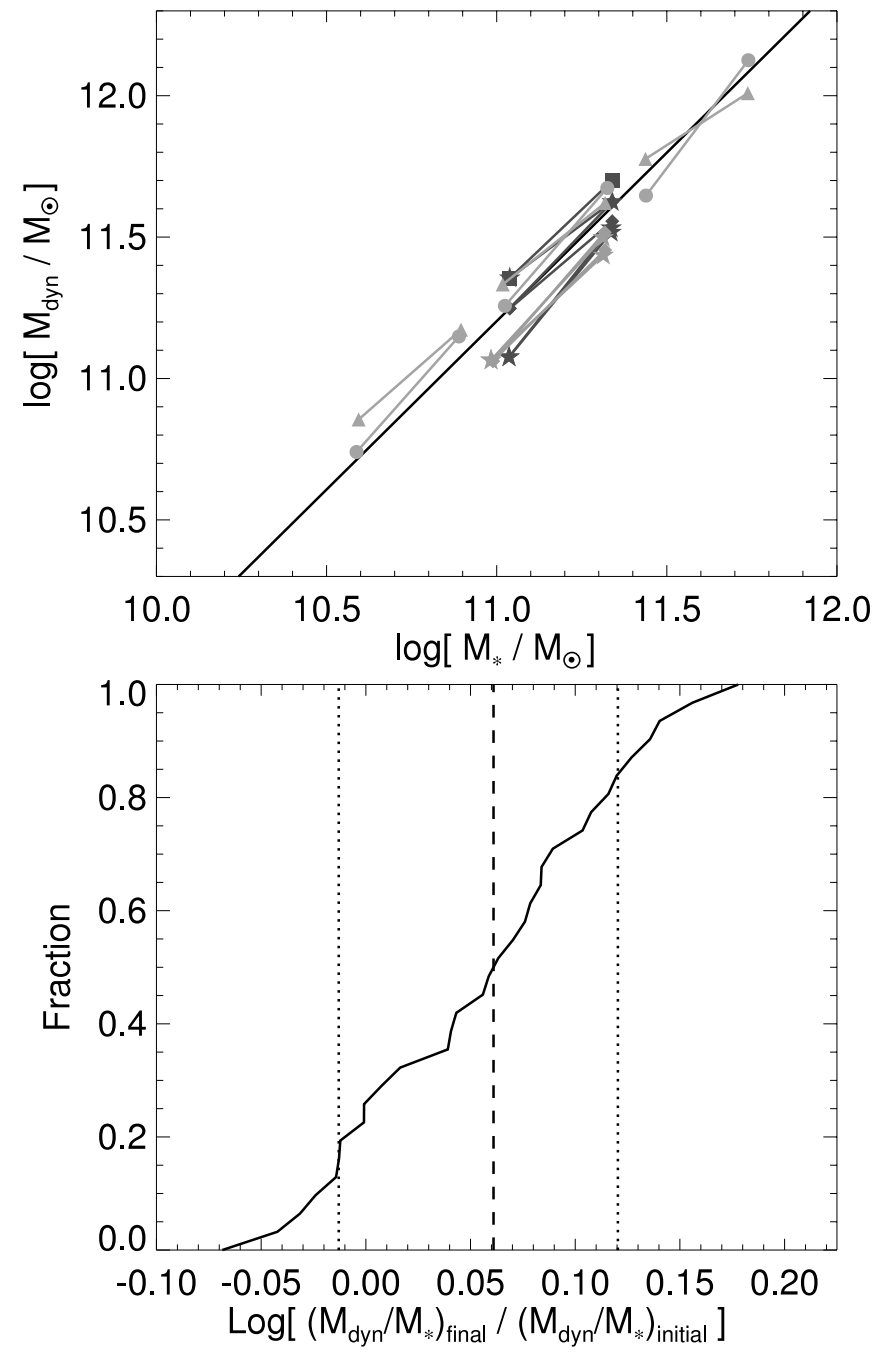

Fig. 23.-Top: Impact of subsequent spheroid-spheroid remergers on the FP. We show the observed FP from Fig. 15 (black line), with the initial and final positions of simulated systems before and after a major remerger ( point style as in Fig. 15; lines connect each remerger to its progenitor). Bottom: Cumulative distribution in relative dynamical-to-stellar mass ratio before and after a major remerger, in our entire ensemble of remerger simulations. Vertical dashed (dotted) lines show the median $( \pm 1 \sigma)$ change in $M_{\text {dyn }} / M_{*}$. Remergers tend to slightly increase $M_{\mathrm{dyn}} / M_{*}$, by almost exactly the amount needed to move parallel to the FP $(0.06 \mathrm{dex})$, but with nonnegligible scatter $(\sim 0.065 \mathrm{dex})$. [See the electronic edition of the Journal for a color version of this figure.]

merger, at a median $r_{f} \approx 2 r_{i}$, the distribution of final radii will be approximately lognormal with scatter $\approx 0.4$ dex (see Paper III). The integrated stellar mass will be constant, but such a scattering will tend to broaden the dark matter distribution in a way that slightly increases the dark matter mass within the stellar $R_{e}$, i.e., scatter some dark matter from near the effective radius of the halo to both smaller and larger radii (total dark matter mass is conserved, but the densities are slightly lower near the halo effective radius, which is much larger than the galaxy effective radius that concerns us here, and slightly higher at much smaller and larger radii). This will slightly raise the central dark matter density, in a way consistent with the observed (weak) change in $M_{\text {dyn }} / M_{*}$ in remergers (effectively mixing slightly more dark matter into the stellar distribution). The net effect of this is that remergers move systems even less with respect to the FP, and dissipation is still the dominant factor setting $M_{\mathrm{dyn}} / M_{*}$.

Figure 23 also demonstrates that although remergers tend, in the mean, to increase $M_{\mathrm{dyn}} / M_{*}$ such that they move parallel to the
FP, there is significant scatter in the change in $M_{\text {dyn }} / M_{*}$ in remergers (in detail, the median increase is $0.06 \mathrm{dex}$, but there is a $1 \sigma$ scatter of $\sim 0.065$ dex about this median). We therefore expect that growing a population of ellipticals by a factor of $\sim 2$ in remergers (equivalent to a single $1: 1$ or three $1: 3$ remergers for most of the objects in the population) will contribute $\sim 0.06 \mathrm{dex}$ intrinsic scatter in $M_{\mathrm{dyn}}\left(M_{*}\right)$. Compared to the intrinsic scatter (in terms of sight line-averaged quantities) of $\sim 0.08 \mathrm{dex}$, this is significant, yielding a final intrinsic scatter of $\sim 0.10$ dex. However, after accounting for the additional $\sim 0.1$ dex sight line-tosight line scatter and observational effects, the contributed scatter is negligible compared to the final observed $\sim 0.18$ dex scatter in $M_{\text {dyn }}\left(M_{*}\right)$. That the observed scatter is not much larger does suggest that there has not been dramatic remerging, but the constraint is weak: it would take $\sim 4-5$ major (mass ratio $1: 1$ ) dry mergers (or $\sim 10$ more likely $1: 3$ dry mergers) to noticeably increase the observed FP scatter at the massive end.

\section{DISCUSSION}

We have developed and implemented a set of observational tests of the theoretical proposal that different degrees of dissipation are responsible for the tilt in the FP (suggested in, e.g., Djorgovski et al. 1988; Kormendy 1989; Bender et al. 1992; Ciotti et al. 1996; developed in numerical simulations in Bekki 1998; Oñorbe et al. 2005; Robertson et al. 2006b; Dekel \& Cox 2006). With measurements of the surface brightness profiles of ellipticals of sufficient accuracy and high dynamic range, we demonstrated in Papers I and III that it is possible to design an empirical decomposition that reliably separates the dissipational (original merger-driven starburst) and dissipationless (scattered stars from the original stellar disks) components of galaxy light profiles. Applying this to a large sample of observed ellipticals, we study here how their FP correlations relate to the mass fractions in the dissipational (or extra light/starburst) component.

We show that systems with larger dissipational fractions have smaller effective radii for their stellar masses and lower ratios of total (stellar plus dark matter) mass to stellar mass within their effective radii (equivalently, lower $M_{\text {dyn }} / M_{*}$ ), i.e., that dissipation can and does move objects onto the FP, in the manner predicted by Robertson et al. (2006b). More important, we show that without the effects of a systematic dependence of the degree of dissipation on mass, there is no significant FP tilt. In other words, systems with the same dissipational/starburst fractions, even over a wide range in mass $(\sim 2-3$ dex $)$, exhibit a constant ratio $M_{\text {true }} \propto$ $M_{*}$. Dissipation not only can tilt systems on the FP, it is required to explain the FP tilt.

The tilt originates because of an expected systematic dependence of dissipational content on mass, arising because lower mass disks (the progenitors of lower mass ellipticals in this scenario) tend to be more gas-rich. Therefore, lower mass systems will, on average, undergo more dissipational mergers. This appears to be true even out to redshifts $z \sim 2-3$, so that the exact formation times of the systems are not especially important. Quantitatively, if we convolve the expected dependence of dissipational fraction on mass (from, e.g., the dependence of gas fraction on disk mass, or from the clear mean systematic trend we see in the fits to the observed ellipticals) with the dependence of $M_{\mathrm{dyn}} / M_{*}$ on dissipational fraction, we obtain a prediction for the FP that is tilted in a manner similar to that observed, $M_{\text {dyn }} \propto M_{*}^{1.2}$.

Of course, merger-induced starbursts may not be the only source of dissipation; for example, stellar mass loss may replenish the gas supply and lead to new dissipational bursts (see, e.g., Ciotti \& Ostriker 2007). Moreover, the merger history and series of induced dissipational events may be more complex than a single or 
couple of idealized major mergers (see, e.g., Kobayashi 2004; Naab et al. 2007). For our purposes, however, all dissipational star formation will appear similar when observed and have the same effects: we are essentially measuring the integrated amount of dissipation and testing the idea that the presence of this dissipation can explain the difference between spheroid and disk scaling relations. In any case, the result is the same, and the agreement with disk gas fractions over the redshift range of interest suggests that star-forming galaxies are viable candidates for the ultimate progenitor systems of ellipticals (however complex the details of the morphological transformation may be). In short, the systematic dependence of the degree of dissipation on mass in elliptical formation is both necessary and sufficient to explain the FP tilt.

\subsection{Possible Sources of Tilt: Why Dissipation Is the Only Viable Possibility}

This conclusion is, to our knowledge, consistent with all existing observational constraints on the FP. We note that repeating our experiments with alternative IMFs affects only the normalizations of the quoted relations; considering alternative stellar evolution models such as those of Leitherer et al. (1999) or Maraston (2005) compared to the default calibration from Bruzual \& Charlot (2003) in Bell et al. (2003) makes no difference, since ellipticals are almost all relatively old and the model differences focus on young stellar populations. Assuming, therefore, that there is not some dramatic error in the stellar population models or the observed effective radii, there are only a limited number of possible explanations for the FP tilt.

Nonhomology.-Technically, this refers to systems being not perfectly self-similar. This must be broken in any model in which there is a dependence or tilt in the physical mass within some radius and the stellar mass $M_{*}$. In practice, the more practical meaning of "homology" is that systems have the same ratio of dynamical mass to true enclosed total mass within their effective radii; i.e., dynamical mass is a good proxy for true mass without a systematic dependence on other galaxy properties. In other words, $M_{\text {true }}=k R_{e} \sigma^{2} / G$, where $k \approx$ constant $\left(M_{\text {true }} \propto R_{e} \sigma^{2} / G\right.$ is expected on dimensional grounds; $k$ is the integral argument that depends on, e.g., the details of profile shape and kinematics). There are two general possibilities:

1. Kinematic nonhomology.-In this case, the meaning of $\sigma$ changes with mass, either because of different contributions of rotationally supported components or because of varying isotropy. Essentially all studies have found that this effect does not contribute to the FP tilt (see, e.g., Gerhard et al. 2001; Riciputi et al. 2005; Cappellari et al. 2007; Nipoti et al. 2008 and references therein), specifically, that there is no correlation between FP residuals and orbital anisotropy or rotation (within the elliptical population). Moreover, Ciotti et al. (1996) demonstrated that reproducing the observed tilt with such trends would require models with internally inconsistent or unphysical orbit structure.

2. Structural nonhomology.-Alternatively, the shape of the light profile could change sufficiently, such that, e.g., even if two systems were both spherical with isotropic velocity dispersion tensors, the integral term relating $M_{\text {true }}$ and $M_{\text {dyn }}$ is significantly different. (Note that this is not really independent of case 1; the dominant effect of a change in the mass profile shape is generally to change the central velocity dispersion, and correspondingly $M_{\text {dyn }}$, rather than dramatically changing, e.g., the physical meaning of $R_{e}$, and differences in mass profile shape will generically require some kinematic nonhomology.) There has been debate regarding this possibility, as some observational studies (e.g., Prugniel \& Simien 1997; Trujillo et al. 2004) have argued that the observed dependence of galaxy Sérsic index on mass is sufficient to drive the tilt in the FP via structural nonhomology. Others have noted, however, that integral models that allow for multiple components or do not explicitly assume a constant $M / L$ with radius do $n o t$ find such nonhomology (e.g., Carollo et al. 1995; Rix et al. 1997; Gerhard et al. 2001; Gerhard 2003; Padmanabhan et al. 2004; Cappellari et al. 2006). Moreover, invoking structural nonhomology can generally explain only differences in central velocity dispersion (the predicted FP tilt from such dynamical models disappears rapidly as $\sigma$ is measured at somewhat larger fractions of $R_{e}$ ); the fact that the FP tilt is not dramatically reduced using velocity dispersions measured in larger apertures (even out to $R_{e}$ ) rules out most classes of such models (Bender et al. 1994; Ciotti et al. 1996; Simien \& Prugniel 1997, 2002; Emsellem et al. 2004; Cappellari et al. 2006; Nipoti et al. 2008).

Our analysis is able to explain both sides of this debate. Fitting galaxy light profiles in a simplified manner, to a single Sérsic index, does appear to yield a significant dependence of Sérsic index on stellar mass, luminosity, or size. In Paper I we demonstrate that this is true of our simulations; i.e., when analyzed in the same manner as the observations, they appear to yield an identical dependence of Sérsic index on mass to that found in Prugniel \& Simien (1997) and Trujillo et al. (2004) (less massive systems, chosen in the same manner as those here to have higher typical dissipational fractions, have lower fitted Sérsic indices over the same observational dynamic range; see also Paper III), even though (at fixed degree of dissipation) the systems are effectively homologous. Why, then, does this occur?

The answer owes to the fact that a single Sérsic index is not a physically robust description of the light profile of an elliptical over its entire extent, and it also fails to include the dark matter distribution. Such a fit mixes the outer dissipationless component of the remnant with the inner, compact starburst remnant. All the observed samples that appear to find a dependence of Sérsic index on mass, when reanalyzed based on our physically motivated twocomponent decomposition, in fact reveal that the outer, dissipationless Sérsic-like component is self-similar (see Papers II and III); what changes with mass or radius is the mass fraction in the central dissipational component. Furthermore, because of the true multicomponent nature of galaxy light profiles, fitting a single Sérsic index yields different answers as a function of the dynamic range employed: for fixed observing conditions, this yields an apparent correlation of Sérsic index with galaxy mass or size (see, e.g., Padmanabhan et al. 2004; Boylan-Kolchin et al. 2005). Because of the role of, e.g., the mass fraction at large radii and subtle differences in the second derivatives of profile shape near $R_{e}$ in driving a fitted Sérsic index, the observed dependence of Sérsic index on mass reflects a complex mix of real differences in galaxy merger history (the fact that low-mass systems have undergone more dissipational mergers, and that high-mass systems are increasingly likely to have undergone subsequent remergers, which will conserve the FP but scatter some small stellar mass to larger radii, leading to larger fitted Sérsic indices) and observational differences in dynamic range, but little significant nonhomology.

We have demonstrated here $(\S 5)$ and in Paper II that differences in the degree of dissipation (i.e., in the strength of the central extra light component), while capable of driving substantial differences in the fitted Sérsic index when the galaxy is fitted to a single Sérsic law, do not in fact drive traditional nonhomology. They are not a large enough fraction of the total mass to dramatically alter the structural integrals. This should not be surprising: the dependence of $M_{\text {true }} / M_{\text {dyn }}$ on Sérsic index (in models where the mass distribution follows a pure Sérsic law) is primarily driven by 
the behavior at large radii; i.e., large Sérsic indices asymptotically approach the power-law behavior $I(r) \propto r^{-2}$, which implies a divergent mass at large $r$ and an implied effective radius $R_{e} \rightarrow \infty$ and rapidly increasing central velocity dispersion. This is precisely where observed profiles are in fact self-similar (in a mean sense; there is considerable variation object to object), with at most a weak dependence on formation history (i.e., median difference $\Delta n_{s} \sim 1$ for those that have undergone major dry mergers, much less than is needed to explain the FP tilt), as we demonstrate in both observations and simulations in Papers II and III.

Further, at these radii, the mass density is increasingly dark matter dominated: the structure integrals only depend significantly on the Sérsic index $n_{s}$ if the dark matter profile is also described by the same Sérsic index, i.e., if the total $M / L$ is a constant function of radius, whereas essentially all kinematic data at large radii indicate that this is not the case (dark matter is required to explain the kinematics at large radii). As we have shown, and as demonstrated in observational kinematic modeling (e.g., Padmanabhan et al. 2004; Cappellari et al. 2006) and lensing studies (Bolton et al. 2007, 2008), the dark matter distribution is relatively insensitive to the details of the stellar mass distribution shape, and therefore the actual nonhomology driven by even large changes in the stellar $n_{s}$ is much less than would be calculated assuming that the dark matter followed the same profile (if it were not so, the observed large dispersion in $n_{s}$ values would necessarily yield much larger scatter about the FP than is observed; see, e.g., Bertin et al. 2002).

The implication that a change in $n_{s}$ should lead to significant structural nonhomology is therefore misleading: the observed dependence of $n_{s}$ on mass owes mainly to issues of finite dynamic range and varying dissipational fractions, none of which give rise to significant structural nonhomology. However, extrapolating to large radii based on these estimated $n_{s}$ values and the incorrect assumptions (1) that a single Sérsic law is a physically meaningful parameterization of the galaxy light profile and (2) that dark matter traces the stellar matter in a strict one-to-one fashion implies (incorrectly) some structural nonhomology.

Moreover, many studies indicate that dynamical mass is proportional to true enclosed mass (without requiring any homology assumption), i.e., that there cannot be a large contribution to tilt from any form of traditional nonhomology, whether kinematic or structural. There are now independent lines of support for this conclusion: Cappellari et al. (2006) (see also van der Marel 1991; Kronawitter et al. 2000; Häring \& Rix 2004) estimate true enclosed masses within $R_{e}$ based on two- and three-integral modeling, from two-dimensional velocity maps of observed ellipticals. Alternatively, Bolton et al. (2007, 2008) measure strong-lensing gravitational masses. In both cases, the authors find $M_{\text {true }} \propto M_{\text {dyn }}$, without any significant dependence on mass; in other words, the FP is unchanged regardless of whether the true total mass enclosed in $R_{e}$ is used, or whether the dynamical mass proxy $M_{\text {dyn }}$ is used. It appears that at most, at the $\sim 3 \sigma$ limits on the observed $M_{\text {true }}-M_{\text {dyn }}$ relation (and based on the fitted $M_{\text {true }}-M_{*}$ relations in Fig. 21), these traditional forms of nonhomology may contribute $\sim \frac{1}{4}$ the observed tilt.

Genuine change in $M / L$.- The observations therefore imply that the FP must reflect a genuine physical difference as a function of mass, namely, that low-mass ellipticals have a higher ratio of stellar or baryonic mass to total (baryonic plus dark matter) mass within the stellar $R_{e}$, relative to high-mass ellipticals. All means of achieving this end can be classified into one of three categories:

1. Varying global baryon fractions.-One could imagine that the stellar and halo mass distributions of different ellipticals are separately self-similar, but that the total ratio of stellar to halo mass changes as a function of galaxy mass. In other words, there are no structural changes implied: one decreases the halo mass relative to galaxy mass in lower mass systems.

While this may seem plausible at the highest galaxy masses (it is well established that in bright clusters, the total stellar-todark matter mass ratio decreases with mass), this is relevant over much larger scales than the stellar $R_{e}$ of the central galaxy. Moreover, over most of the mass range of interest, the known trends in global stellar-to-dark matter fractions are opposite to that needed to explain the FP tilt.

At masses below $\sim M_{*}$, where the FP is observed to be continuous (recall that the observed FP and its tilt extend to systems with masses $<10^{-2} M_{*}$ ), all $\Lambda \mathrm{CDM}$ models (e.g., Conroy et al. 2006; Zentner et al. 2005; Zheng et al. 2005; Vale \& Ostriker 2006; Shankar et al. 2006; van den Bosch et al. 2007) and observational constraints (Eke et al. 2004; Yang et al. 2005; Mandelbaum et al. 2006; Weinmann et al. 2006; Wang et al. 2006) require that the ratio of total dark matter halo mass to stellar mass is higher in lower mass systems, i.e., that star formation is less efficient in low-mass systems. This is contrary to the effect desired here and demonstrates that the FP tilt cannot owe to a simple global change in baryon fraction. Indirectly, however, the cosmological trend of stellar to dark matter mass is in fact important: this lower star formation efficiency in low-mass systems means that they have larger gas fractions when they undergo mergers, which we show does give rise to the FP tilt.

If the global baryonic mass ratio is unchanging at small radii (or changes in the opposite sense needed to tilt the FP), then the only other possibility is that the size/shape of the halo and stellar distributions vary relative to one another; i.e., one of the two components is made more or less compact, relative to the other, altering the ratio of stellar to total mass within the stellar $R_{e}$. Recall that the radii of interest are small relative to the halo virial radii and contain only a small fraction of the total halo dark matter mass, so regardless of the total halo-to-stellar mass ratio, changing the compactness of one component relative to the other can make a significant difference. There are two possibilities for this relative contraction/expansion.

2. Baryons are fixed, halos contract.-In this scenario, the galaxy stellar mass distributions are "scale-free" (i.e., do not change owing to external factors), but the dark matter halos are less compact in low-mass ellipticals, so that within the stellar $R_{e}$ (i.e., the central regions of the halo) the total dark matter mass fraction enclosed is lower. This is quickly ruled out: all cosmological models (e.g., Navarro et al. 1996; Bullock et al. 2001; Wechsler et al. 2002; Dolag et al. 2004; Kuhlen et al. 2005; Neto et al. 2007) and observations from, e.g., weak lensing and X-ray mass measurements (Buote et al. 2007; Schmidt \& Allen 2007; Comerford \& Natarajan 2007) find that halo concentration is a weak, decreasing function of galaxy mass, the opposite of the effect desired.

Furthermore, if this were the case, one would expect to see it in progenitor disks as well (the halos are insensitive to the morphological transformation of their central galaxies); however, the baryonic Tully-Fisher relation and constraints on the dark matter halos of disks (e.g., Persic \& Salucci 1990; Persic et al. 1996b; Bell \& de Jong 2001; McGaugh 2005 and references therein) reflect the expected cosmological trends (namely, baryon fractions and halo sizes scaling as predicted in $\Lambda$ CDM, in a weaker and opposite sense from that necessary if the FP tilt were to be explained in this manner). In short, effects 1 and 2 would predict that disks should follow the same FP scalings as ellipticals (modulo possible normalization offsets), while in fact they obey different scalings 


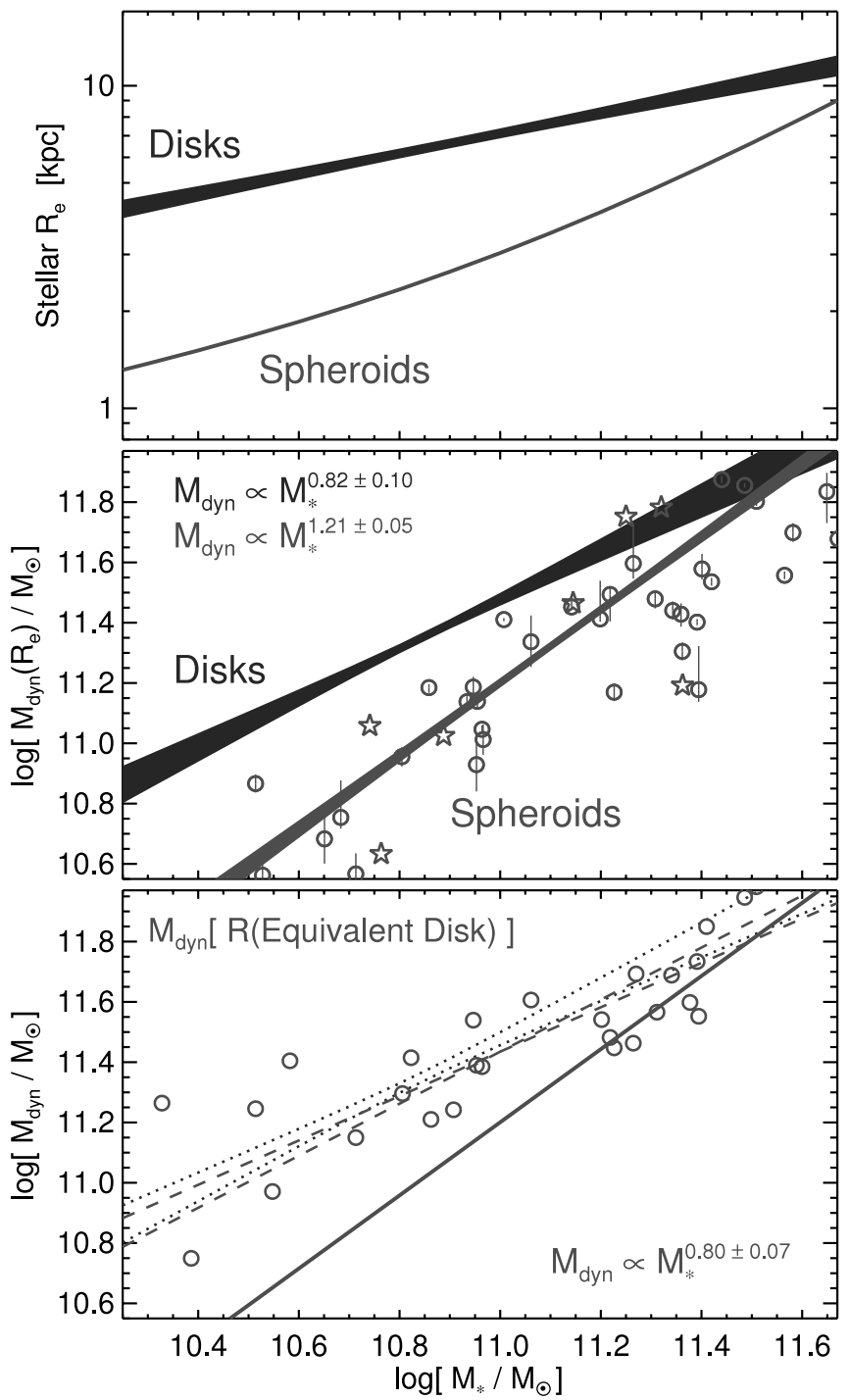

FIG. 24.-Top: Mean stellar size-mass relation of ellipticals, from Fig. 19, compared to that of disks (from Shen et al. 2003). Middle: FP of spheroids and disks, i.e., ratio of $M_{\text {dyn }}$ (evaluated at $R_{e}$ ) to $M_{*}$. We show the observed ellipticals from Fig. 15 and the best-fit power law $M_{\text {dyn }} \propto M_{*}^{1+\alpha}$ to both relations (with corresponding uncertainties). Disk correlation is from the data in Bell \& de Jong (2001). At a given stellar mass, (low mass) spheroids have more compact stellar mass distributions and less total mass $\left(M_{\mathrm{dyn}}\right)$ enclosed within their stellar $R_{e}$. Bottom: Same as the middle panel, but measuring $M_{\text {dyn }}$ for the same observed ellipticals (from the middle panel) at the mean expected radius of an equivalent (similar stellar mass) disk (from the top panel). The best-fit relation to these data is shown; it is indistinguishable from the relation for disks. At the radii of their equivalent disks, ellipticals and disks have the same enclosed total (dark matter plus stellar) masses. The distribution of dark matter is not significantly different: the smaller sizes and $M_{\text {dyn }}$ of ellipticals must reflect a contraction of the baryonic material relative to the dark matter, as predicted to occur in dissipative mergers. [See the electronic edition of the Journal for a color version of this figure.]

with, in many cases, an opposite qualitative sense (e.g., their sizemass, velocity-mass, surface brightness-mass, and FP scalings; Faber \& Jackson 1976; Kormendy 1989; Shen et al. 2003).

Figure 24 demonstrates this explicitly. We show the stellar sizemass relation of ellipticals (from Fig. 19), compared to that of disks (from Shen et al. 2003; but for our purposes here different sources agree well). Modulo a small normalization offset (owing to the profile shape), ellipticals would necessarily obey the same correlation if they were formed in purely dissipationless mergers (see also Ciotti \& van Albada 2001). Obviously, ellipticals at low mass (where we empirically estimate and theoretically expect dissipation to be important) are much more compact in their stellar distributions than disks. We also compare the FP of both types of objects, i.e., dynamical mass $M_{\text {dyn }}$ (measured within $R_{e}$ of the stellar light) versus stellar mass. We estimate the relation for disks based on the data and best-fit relations in Bell \& de Jong (2001) (see also Persic \& Salucci [1990] and Persic et al. [1996b], who construct a similar correlation). Note that, for our purposes here, it makes little difference whether we plot the baryonic or stellar mass (there is almost no difference for ellipticals, owing to their small gas content, and for disks, the relations fall within the quoted uncertainties in either case). It also makes no significant difference whether we use the same virial constant $k$ to estimate $M_{\text {dyn }}$ for both disks and ellipticals, or attempt to make some correction for either profile shapes or the use of a velocity dispersion as opposed to a circular velocity (the difference is small, a factor $\lesssim 2$, and we are ultimately interested in the qualitative scalings).

It is clear (in agreement with previous work) that the scaling of $M_{\text {dyn }} / M_{*}$ in disks is opposite that of ellipticals: there is either no tilt or inverse tilt in the FP (i.e., $M_{\mathrm{dyn}} / M_{*}$ is the same or higher in low-mass disks). For ellipticals where we have kinematic data as a function of radius, we can test whether the difference in the disk and elliptical scalings owes to effect 1 or effect 2 ; we do so by evaluating their enclosed mass $M_{\text {dyn }}$ not at the observed stellar effective radius of the spheroid, but at the radius of an equivalent disk (i.e., at the mean $R_{e}$ of a disk of the same stellar mass). The resulting trend of $M_{\text {dyn }}$ versus $M_{*}$ is indistinguishable from that of observed disks; i.e., by considering elliptical properties at their equivalent disk radii (radii they would have in the absence of dissipation), we effectively remove the tilt of the FP and recover the observed correlations of disks.

At the radius of a disk of similar mass (equivalently, at the radius the elliptical would have, if it were the product of a purely dissipationless merger of stellar disks), ellipticals have the same enclosed total mass as equivalent disks. In other words, at the same (equivalent dissipationless) radius, disks and ellipticals of the same mass have the same dark matter mass content and distribution. In general, it is observationally well established that the FP correlations of ellipticals become less distinct from those of disks as their properties are measured at larger radii. These correlations clearly rule out scenarios 1 and 2 above: if case 2 were true, disks and ellipticals should obey a similar stellar size-mass relation, and at the $R_{e}$ of an equivalent disk, ellipticals should still have much lower $M_{\text {dyn }}$ than spirals. If case 1 were true (i.e., both stellar and dark matter were more compact in ellipticals, but with lower total dark matter-to-stellar mass ratios), then we would again expect much lower $M_{\text {dyn }}$ at the equivalent disk radius in ellipticals.

3. Halos are fixed, baryons contract.-The only remaining possibility is our conclusion in this paper, namely, that the FP tilt arises because lower mass spheroids have more compact stellar mass distributions, relative to their halos (equivalently, relative to what their stellar mass distribution would be in the absence of dissipation). We have demonstrated that this outcome is a natural consequence of dissipation in mergers: regardless of the initial scalings obeyed by progenitor disks, the fact that low-mass disks are more gas-rich implies that, on average, there will be more dissipation in their mergers, yielding more compact baryonic remnants (while having little effect on the halo), and therefore increasing the ratio of stellar to dark matter mass inside the stellar $R_{e}$ in lower mass systems. We further demonstrate that this reproduces precisely the observed tilt and scalings of elliptical properties with mass, while being consistent with all other observational constraints on the FP, including the "homology constraint," that $M_{\text {true }} \propto M_{\text {dyn }}$. 
We have also shown that dissipation is necessary in achieving this. It has been known for some time that dissipationless mergers cannot alter the phase-space density of ellipticals relative to their disk progenitors; consequently, ellipticals produced through dissipationless mergers obey the same scaling relations as their spiral progenitors (modulo normalization offsets), in stark contrast to the observed FP scalings. We demonstrate this explicitly: systems with the same dissipational fraction have the same ratio of total to stellar mass within $R_{e}$, i.e., do not internally exhibit any FP tilt. Furthermore, we show that dissipation is the dominant factor determining the effective radii of ellipticals at fixed mass, even allowing for differences in formation and merger history; therefore, if the explanation for the FP invokes any systematic change in elliptical sizes, it must involve dissipation. Only when the observed dependence of dissipation on mass is included is the observed tilt recovered.

\subsection{Additional Predictions}

We have extensively considered the role of dissipation in setting the FP tilt, effectively changing the ratio of $M_{\text {dyn }} / M_{*}$. To the extent that other properties also trace the degree of dissipation, we predict that these should similarly correlate with $M_{\text {dyn }} / M_{*}$. In Papers I and II we develop an extensive set of predictions for elliptical properties that relate to the degree of dissipation in the spheroid-forming merger and show how these relate to, e.g., the observed extra light (i.e., the tracer of the degree of dissipation in the spheroid-forming merger-induced starburst). We refer to those papers for details and a large number of observational proxies of the degree of dissipation that can be used to further test the ideas herein.

To the extent that the degree of dissipation in the original spheroid-forming merger reflects the gas fractions of the progenitor disks, it must also reflect the progenitor star formation history. Broadly speaking, disks with more extended star formation histories would be expected to have larger gas fractions at the time of their merger, with younger stellar population ages and lower $\alpha$-enhancement. If we ignore the effect of the merger on these stellar populations (a reasonable assumption if the system is observed at times significantly later than the merger, and if the mass fraction formed in the merger-induced starburst is not large, which are true for most of the observed systems of interest here), then these should be reflected in the stellar populations of the elliptical remnant. Preliminary observational comparisons from J. Graves et al. (2008, in preparation) appear to support these predictions and can provide powerful independent tests and constraints for models of dissipation in spheroid formation: we therefore outline some relevant quantitative predictions.

Consider the following highly simplified toy model: identical progenitor disks with initial gas fraction $f_{\text {gas }}=1$ follow an exponential, closed-box star formation history with timescale $\tau$, i.e., $\dot{M}_{*} \propto \exp (-t / \tau)$, and merge at time $t_{m}$, when the remaining gas is rapidly consumed in a central starburst. The gas fraction at the time of the merger (and correspondingly, the dissipational fraction in the starburst) will be $f_{\mathrm{sb}}=\exp \left(-t_{m} / \tau\right)$ [giving $\left.\tau=t_{m} / \ln \left(1 / f_{\mathrm{sb}}\right)\right]$, and the mass-weighted mean formation time of the stars will be $t_{\text {form }}=\tau\left[1-\exp \left(-t_{m} / \tau\right)\right]=t_{m}\left(1-f_{\mathrm{sb}}\right) / \ln \left(1 / f_{\mathrm{sb}}\right)$. For systems with a similar redshift range of their last major merger (similar $t_{m}$, expected for systems of similar mass), their stellar formation times should therefore correlate with the starburst fraction $f_{\mathrm{sb}}$ (both depending implicitly on the premerger star formation timescale $\tau$ ).

We have shown in $\S 6$ how $M_{\text {dyn }} / M_{*}$ is predicted to scale with the dissipational fraction $f_{\mathrm{sb}}$ [this can be roughly approximated as $M_{\mathrm{dyn}} / M_{*} \sim\left(f_{\mathrm{sb}} / 0.2\right)^{-1 / 2}$ over the range of interest]; combin- ing the two, this yields an expected correlation between formation time and $x \equiv M_{\mathrm{dyn}} / M_{*}$ of the form $t_{\mathrm{form}}=0.5 t_{m}\left(1-0.2 x^{-2}\right) /$ $\ln (2.24 x)$. Observed at $z=0$ (i.e., with age $\left.t_{\mathrm{H}}-t_{\text {form }}\right)$, ellipticals with larger $x=M_{\mathrm{dyn}} / M_{*}$ at fixed mass (similar $t_{m}$ ) should be older: for a typical $t_{m} \sim 10 \mathrm{Gyr}$, systems with $M_{\text {dyn }} / M_{*} \approx 2$ are predicted to be $\sim 2$ Gyr older than systems with $M_{\text {dyn }} / M_{*} \approx 1$.

A shorter star formation history also implies higher $\alpha$-enrichment in the progenitors. If we adopt the correlation for simple star formation models in Thomas et al. (2005), $[\alpha / \mathrm{Fe}] \approx \frac{1}{5}-\frac{1}{6} \log \Delta t$ (where $\Delta t$ is the star formation timescale for a Gaussian burst, but for our purposes here can be replaced by $\tau$ modulo a conversion constant), and the scalings above, then we obtain the result (independent of the merger time $t_{m}$ ) that ellipticals with higher $M_{\text {dyn }} / M_{*}$ should be more $\alpha$-enriched. Specifically, objects with $M_{\text {dyn }} / M_{*} \approx 2$ are predicted to have $[\alpha / \mathrm{Fe}]$ values $\approx 0.04-0.05$ higher than systems with $M_{\mathrm{dyn}} / M_{*} \approx 1$.

Predictions for the absolute metallicities are more ambiguous (but see Paper II). In general, the trend of total/mean metallicity with mass will be dominated by the metallicity of premerger disks, which observations show (excluding the most gas-rich disks, where self-enrichment in the merger will dominate the final total metallicity) trace a similar mass-metallicity correlation to ellipticals (Gallazzi et al. 2005). In the absence of outflows or recycling, the metallicity would be the same for any systems with the same total accreted gas content and stellar mass (with no dependence on the "starburst" content at fixed mass). However, to the extent that outflows in low-mass systems are responsible for the massmetallicity relation (as is generally believed), the detailed interplay between these outflows and merger-induced starbursts will be important. In broad terms, if outflow strengths and velocities are similar, then we expect dissipational star formation at the center of the galaxy to retain a higher metal content in comparison to the same star formation in a more extended disk (since escape velocities from the galactic center and densities leading to radiative losses in the outflows are higher). In this case, at fixed mass, more dissipational (lower $M_{\text {dyn }} / M_{*}$, higher surface brightness) systems should have slightly higher metallicities than their less dissipational counterparts (and, as demonstrated in Paper I, this should be correlated with the effects above: at fixed mass, the dependence of both quantities on dissipation should give rise to an inverse correlation between metallicity and stellar population age or $\alpha$-enrichment). Experimenting with, e.g., different degrees of dissipation, outflow strengths, and initial disk metallicities in our simulations, we estimate this to be a relatively small ( $\sim 0.1 \mathrm{dex})$ effect, not sufficient to dramatically affect the global mass-metallicity relation, but potentially visible in detailed studies.

There is one important caveat here: systems of the same mass might also have had more gas-rich progenitors because they formed from mergers at very early times (i.e., had a different merger time $t_{m}$, in the toy model illustrated above), making them older and more $\alpha$-enriched. However, cosmological estimates (e.g., Hopkins et al. 2008b) suggest that this process is not dominant at a given stellar mass; i.e., systems of comparable stellar mass (and correspondingly similar total halo masses) tend to have similar merger histories. Specifically, the relatively large scatter in star formation history and disk gas fractions at fixed mass and redshift (a factor of $\sim 2$ in $f_{\text {gas }}$ ) is larger than the scatter introduced by the combination of a scatter in formation times and the systematic cosmological evolution of disk gas fractions with time. Furthermore, systems with such early mergers will usually have multiple subsequent mergers at later times, so they will grow significantly in mass and have their effective radii substantially modified by these additional processes (such that they should and will be compared to different systems at $z=0$ ). 
Considering higher order effects, we demonstrate in Paper II that the strength of stellar population gradients is correlated with the degree of dissipation in the original spheroid-forming gas-rich merger, and we show in Paper III that this holds even for remnants of subsequent gas-poor dry remergers. The most useful gradients in this sense are metallicity gradients: stellar age gradients and (especially) color gradients evolve strongly with time even in a fixed, passively evolving elliptical (owing to the change in relative $M / L$ for young and old stellar populations) and as such are more ambiguous, and gradients in $\alpha$-enhancement are more sensitive to the gradients and overall star formation histories in the premerger disks. Metallicity gradients are, on the other hand, generally dominated by the degree of dissipation and imprinted in the gas-rich merger, and they are not sensitive to the star formation history of the premerger disks, making them a more robust diagnostic for our purposes. At fixed mass, stronger gradients indicate more dissipation, and so we predict that, at fixed mass, ellipticals with higher $M_{\text {dyn }} / M_{*}$ should have weaker metallicity gradients (see Paper II).

\subsection{Summary}

We have demonstrated from observations that the tilt of the FP owes to differential degrees of dissipation as a function of mass. Lower mass disks are more gas-rich, so their mergers are more dissipational: a larger fraction of the remnant mass is formed in a dissipational, merger-induced compact central starburst in the final stages of a major merger. This yields a remnant with a more compact stellar mass distribution, i.e., smaller $R_{e}$ relative to their progenitor disks, in lower mass ellipticals. The dark matter distribution is only weakly affected, implying that the stellar distribution in lowmass ellipticals is more compact, relative to the dark matter, than in equivalent disks or higher mass ellipticals. Consequently, relatively less dark matter mass is enclosed within the stellar effective radius in low-mass ellipticals, so the ratio of enclosed mass $M_{\mathrm{dyn}} / M_{*}$ is an increasing function of mass $\left(M_{\mathrm{dyn}} / M_{*} \propto M_{*}^{\alpha}\right)$. This is the "tilt" in the FP. Given the observed, quantitative dependence of gas fractions on mass, the tilt is predicted to be exactly that observed, $\alpha \approx 0.2$.

Using a new empirical method, we robustly estimate the amount of dissipation involved in the formation of a given elliptical. Specifically, with data of sufficient quality, we separate the observed surface brightness profile into an outer, violently relaxed component, which was established in a dissipationless manner, and an inner starburst remnant or extra light component. We demonstrate in Papers I, II, and III that observations of both evolved ellipticals and recent merger remnants support the proposal that this compact nuclear mass component is a good proxy for the true mass formed in a dissipational starburst. Using this proxy, we show that the observed sizes of ellipticals, at fixed mass, depend strongly on the degree of dissipation involved in their formation (more so than even, e.g., the number of mergers in their formation history). Correspondingly, we show that the ratio of total to stellar mass within the stellar effective radius, $M_{\mathrm{dyn}} / M_{*}$, is a function of dissipation, both globally and at fixed mass (in the sense that elliptical sizes and $M_{\mathrm{dyn}} / M_{*}$ are decreasing functions of the amount of dissipation). These observed dependencies are highly significant $\left(P_{\text {null }} \lesssim 10^{-7}\right)$. Motivated by this, we show that by removing the mean systematic dependence of dissipation on mass, we can empirically remove the tilt of the FP. Considering ellipticals with the same dissipational extra light fractions, we show that they obey a relation $M_{\text {dyn }} \propto M_{*}$ (i.e., ellipticals with the same extra light content have the same ratio of dynamical to stellar mass within $R_{e}$, independent of mass).

In the proposed dissipational models of the FP (Bekki 1998; Oñorbe et al. 2005; Robertson et al. 2006b; Dekel \& Cox 2006), the tilt of the FP and its projected correlations (e.g., the steepness of the stellar size-mass correlation of ellipticals relative to that of disks) arise because low-mass disks are more gas-rich, and therefore low-mass mergers and ellipticals will have (on average) systematically higher degrees of dissipation and therefore smaller (relative) $R_{e}$ and $M_{\mathrm{dyn}} / M_{*}$. If we consider, e.g., simulations that obey the observed correlation between disk gas content and mass (as opposed to being dissipationless, or having all the same gas fractions independent of mass, neither of which is consistent with observations), then the FP predicted has exactly the observed tilt. Equivalently, the observed mean dissipational fractions of ellipticals, as a function of mass, agree well with the observed gas fractions of progenitor disks of the same masses, over the redshift range $z \sim 0-2$. In other words, dissipation is both necessary and sufficient to explain the FP tilt and differences between disk and elliptical scaling relations.

To our knowledge, this is the first explicit observational test of these theoretical models. We further demonstrate that other mechanisms cannot be responsible for the majority of the FP tilt. For example, observations have demonstrated that the "homology assumption," namely, that $M_{\text {true }} \propto \sigma^{2} R_{e}$, is valid, and we show that simulations predict this: the homology breaking introduced by dissipation is negligible. In other words, the FP tilt reflects the ratio of stellar to true mass enclosed within $R_{e}: M_{\text {true }} / M_{*} \propto M_{*}^{\alpha}$ (this ratio is not constant), rather than an "apparent" effect. We also show that, if we measure elliptical properties at the radius of an equivalent disk, the tilt of the FP is removed: within the radii of disks of the same mass, ellipticals have the same total and dynamical masses. That is, the dependence of $M_{\mathrm{dyn}} / M_{*}$ cannot be driven primarily by changes in the dark matter distribution at fixed baryonic properties, nor by changes in the total dark matter-tostellar mass ratio (integrated over the entire halo). The variation in $M_{\mathrm{dyn}} / M_{*}$ within $R_{e}$ must predominantly reflect the change in size of the baryonic component: low-mass ellipticals have much more compact stellar distributions than similar-mass disks and therefore have less enclosed dark matter within that stellar $R_{e}$, as predicted in dissipational theories.

Together, these observational tests represent an important vindication of the "merger hypothesis," that ellipticals are formed by the gas-rich mergers of disk galaxies, and models for the origin of the FP in dissipational major mergers (Robertson et al. 2006b; Dekel \& Cox 2006). We explicitly demonstrate that, regardless of subsequent gas-poor (spheroid-spheroid or "dry") remergers, the location of systems with respect to the FP and, e.g., elliptical sizemass and velocity dispersion-mass relations is primarily determined by the amount of dissipation involved in their formation, i.e., the gas content involved in the original, spheroid-forming merger. Gas-rich mergers cannot be ignored in the formation of ellipticals. We have demonstrated not only that the FP is consistent with the merger hypothesis, but that (given the systematic dependence of disk gas fractions on mass) an FP tilted in the manner observed is a necessary prediction of the theory.

We have also shown that elliptical sizes, inferred dissipational fractions, and the FP are completely consistent with the formation of ellipticals in mergers of disks with similar properties (sizes, gas fractions, dark matter halo masses and sizes) to those observed in low-redshift $(z \sim 0-1)$ disks. In other words, the sizes of ellipticals and their FP correlations do not require elliptical progenitors to be more compact than observed, low-redshift disks. Dissipation is sufficient to explain the differences in their densities and sizes. The fact that, within the radius of an equivalent disk, ellipticals obey the same correlation between total and stellar mass actually implies that their dark matter halos (and presumably those of their 
progenitors) are not significantly more compact than those of lowredshift disks.

This is important for the viability of the merger hypothesis, given the observations indicating that disk and halo sizes do not evolve strongly with redshift (Ravindranath et al. 2004; Barden et al. 2005; Trujillo et al. 2006; Zirm et al. 2007). This is not to say that elliptical sizes might not evolve with redshift (which is easily possible if, e.g., disk gas fractions systematically evolve; see Khochfar \& Silk 2006; Hopkins et al. 2007a), nor that ellipticals all formed at low redshift (indeed, if the disk size evolution is weak, then ellipticals could rapidly form at relatively early times and still resemble the products of low-redshift disks). However, it does imply that exotic progenitors (progenitors not found in the local universe) are not required to explain the observed correla- tions, surface brightness profiles (see Papers II and III), or kinematics (see Cox et al. 2006b) of typical local ellipticals.

We thank John Kormendy, Tod Lauer, and Barry Rothberg for providing observations and suggestions for the content herein, and we thank Brant Robertson, Marijn Franx, and Sandy Faber for helpful discussions throughout the development of this paper. We also thank our referee, Luca Ciotti, for helpful advice on the content and discussion herein. This work was supported in part by NSF grants ACI 96-19019, AST 00-71019, AST 02-06299, and AST 03-07690 and NASA ATP grants NAG5-12140, NAG513292, and NAG5-13381. Support for T. J. C. was provided by the W. M. Keck Foundation.

\section{REFERENCES}

Avila-Reese, V., Zavala, J., Firmani, C., \& Hernández-Toledo, H. M. 2008, AJ, 136,1340

Balcells, M., Graham, A. W., \& Peletier, R. F. 2007, ApJ, 665, 1084

Barden, M., et al. 2005, ApJ, 635, 959

Barnes, J. E. 1988, ApJ, 331, 699

Barnes, J. E., \& Hernquist, L. 1996, ApJ, 471, 115

Barnes, J. E., \& Hernquist, L. E. 1991, ApJ, 370, L65

Bekki, K. 1998, ApJ, 496, 713

Bell, E. F., \& de Jong, R. S. 2000, MNRAS, 312, 497 2001, ApJ, 550, 212

Bell, E. F., McIntosh, D. H., Katz, N., \& Weinberg, M. D. 2003, ApJS, 149, 289

Bender, R. 1988, A\&A, 193, L7

Bender, R., Burstein, D., \& Faber, S. M. 1992, ApJ, 399, 462 1993, ApJ, 411, 153

Bender, R., Doebereiner, S., \& Moellenhoff, C. 1987, A\&A, 177, L53 1988, A\&AS, 74, 385

Bender, R., Saglia, R. P., \& Gerhard, O. E. 1994, MNRAS, 269, 785

Bernardi, M., et al. 2003, AJ, 125, 1849

Bertin, G., Ciotti, L., \& Del Principe, M. 2002, A\&A, 386, 149

Binggeli, B., Sandage, A., \& Tammann, G. A. 1985, AJ, 90, 1681

Bolton, A. S., Burles, S., Treu, T., Koopmans, L. V. E., \& Moustakas, L. A. 2007, ApJ, 665, L105

Bolton, A. S., Treu, T., Koopmans, L. V. E., Gavazzi, R., Moustakas, L. A.,

Burles, S., Schlegel, D. J., \& Wayth, R. 2008, ApJ, 684, 248

Borriello, A., \& Salucci, P. 2001, MNRAS, 323, 285

Borriello, A., Salucci, P., \& Danese, L. 2003, MNRAS, 341, 1109

Boylan-Kolchin, M., Ma, C.-P., \& Quataert, E. 2005, MNRAS, 362, 184 2006, MNRAS, 369, 1081

Bruzual, G., \& Charlot, S. 2003, MNRAS, 344, 1000

Bullock, J. S., Kolatt, T. S., Sigad, Y., Somerville, R. S., Kravtsov, A. V., Klypin, A. A., Primack, J. R., \& Dekel, A. 2001, MNRAS, 321, 559

Buote, D. A., Gastaldello, F., Humphrey, P. J., Zappacosta, L., Bullock, J. S., Brighenti, F., \& Mathews, W. G. 2007, ApJ, 664, 123

Busha, M. T., Evrard, A. E., Adams, F. C., \& Wechsler, R. H. 2005, MNRAS, 363, L11

Caon, N., Capaccioli, M., \& D’Onofrio, M. 1994, A\&AS, 106, 199

Caon, N., Capaccioli, M., \& Rampazzo, R. 1990, A\&AS, 86, 429

Capelato, H. V., de Carvalho, R. R., \& Carlberg, R. G. 1995, ApJ, 451, 525

Cappellari, M., et al. 2006, MNRAS, 366, 1126 . 2007, MNRAS, 379, 418

Carlberg, R. G. 1986, ApJ, 310, 593

Carollo, C. M., de Zeeuw, P. T., van der Marel, R. P., Danziger, I. J., \& Qian, E. E. 1995, ApJ, 441, L25

Chabrier, G. 2003, PASP, 115, 763

Ciotti, L., Lanzoni, B., \& Renzini, A. 1996, MNRAS, 282, 1

Ciotti, L., Lanzoni, B., \& Volonteri, M. 2007, ApJ, 658, 65

Ciotti, L., \& Ostriker, J. P. 2007, ApJ, 665, 1038

Ciotti, L., \& van Albada, T. S. 2001, ApJ, 552, L13

Comerford, J. M., \& Natarajan, P. 2007, MNRAS, 379, 190

Conroy, C., Wechsler, R. H., \& Kravtsov, A. V. 2006, ApJ, 647, 201

Côté, P., et al. 2006, ApJS, 165, 57

Courteau, S., Dutton, A. A., van den Bosch, F. C., MacArthur, L. A., Dekel, A., McIntosh, D. H., \& Dale, D. A. 2007, ApJ, 671, 203

Covington, M., Dekel, A., Cox, T. J., Jonsson, P., \& Primack, J. R. 2008, MNRAS, 384, 94

Cox, T. J., Di Matteo, T., Hernquist, L., Hopkins, P. F., Robertson, B., \& Springel, V. 2006a, ApJ, 643, 692
Cox, T. J., Dutta, S. N., Di Matteo, T., Hernquist, L., Hopkins, P. F., Robertson, B., \& Springel, V. 2006b, ApJ, 650, 791

Dantas, C. C., Capelato, H. V., Ribeiro, A. L. B., \& de Carvalho, R. R. 2003, MNRAS, 340, 398

Davé, R., Hernquist, L., Katz, N., \& Weinberg, D. H. 1999, ApJ, 511, 521

Davis, L. E., Cawson, M., Davies, R. L., \& Illingworth, G. 1985, AJ, 90, 169

Dekel, A., \& Cox, T. J. 2006, MNRAS, 370, 1445

de Lucia, G., \& Blaizot, J. 2007, MNRAS, 375, 2

Desroches, L.-B., Quataert, E., Ma, C.-P., \& West, A. A. 2007, MNRAS, 377 , 402

de Vaucouleurs, G. 1948, Ann. d'Astrophys., 11, 247

Di Matteo, T., Springel, V., \& Hernquist, L. 2005, Nature, 433, 604

di Serego Alighieri, S., et al. 2005, A\&A, 442, 125

Djorgovski, S., \& Davis, M. 1987, ApJ, 313, 59

Djorgovski, S., de Carvalho, R., \& Han, M.-S. 1988, in ASP Conf. Ser. 4, The Extragalactic Distance Scale, ed. S. van den Bergh \& C. J. Pritchet (San Francisco: ASP), 329

Dolag, K., Bartelmann, M., Perrotta, F., Baccigalupi, C., Moscardini, L., Meneghetti, M., \& Tormen, G. 2004, A\&A, 416, 853

Doyon, R., Wells, M., Wright, G. S., Joseph, R. D., Nadeau, D., \& James, P. A. 1994, ApJ, 437, L23

Dressler, A., Lynden-Bell, D., Burstein, D., Davies, R. L., Faber, S. M., Terlevich, R., \& Wegner, G. 1987, ApJ, 313, 42

Eke, V. R., et al. 2004, MNRAS, 355, 769

Emsellem, E., et al. 2004, MNRAS, 352, 721 2007, MNRAS, 379, 401

Erb, D. K., Steidel, C. C., Shapley, A. E., Pettini, M., Reddy, N. A., \& Adelberger, K. L. 2006, ApJ, 646, 107

Faber, S. M., \& Jackson, R. E. 1976, ApJ, 204, 668

Faber, S. M., Tremaine, S., Ajhar, E. A., Byun, Y.-I., Dressler, A., Gebhardt, K., Grillmair, C., Kormendy, J., Lauer, T. R., \& Richstone, D. 1997, AJ, 114, 1771

Ferrarese, L., et al. 2006, ApJS, 164, 334

Gallazzi, A., Charlot, S., Brinchmann, J., \& White, S. D. M. 2006, MNRAS, 370,1106

Gallazzi, A., Charlot, S., Brinchmann, J., White, S. D. M., \& Tremonti, C. A. 2005, MNRAS, 362, 41

Genzel, R., Tacconi, L. J., Rigopoulou, D., Lutz, D., \& Tecza, M. 2001, ApJ, 563,527

Gerhard, O. 2003, in The Mass of Galaxies at Low and High Redshift, ed. R. Bender \& A. Renzini (Berlin: Springer), 62

Gerhard, O., Kronawitter, A., Saglia, R. P., \& Bender, R. 2001, AJ, 121, 1936

Goto, T. 2005, MNRAS, 357, 937

Gunn, J. E. 1987, in Nearly Normal Galaxies: From the Planck Time to the Present, ed. S. M. Faber (New York: Springer), 455

Häring, N., \& Rix, H.-W. 2004, ApJ, 604, L89

Hausman, M. A., \& Ostriker, J. P. 1978, ApJ, 224, 320

Hernquist, L. 1989, Nature, 340, 687 1990, ApJ, 356, 359 1993, ApJ, 404, 717

Hernquist, L., Spergel, D. N., \& Heyl, J. S. 1993, ApJ, 416, 415

Heymans, C., et al. 2006, MNRAS, 371, L60

Hibbard, J. E., \& Yun, M. S. 1999, ApJ, 522, L93

Hopkins, P. F., Cox, T. J., Dutta, S. N., Hernquist, L., Kormendy, J., \& Lauer, T. R. 2008a, ApJ, submitted (arXiv:0805.3533) (Paper II)

Hopkins, P. F., Cox, T. J., Kereš, D., \& Hernquist, L. 2008b, ApJS, 175, 390

Hopkins, P. F., Cox, T. J., Younger, J. D., \& Hernquist, L. 2008c, ApJ, submitted (arXiv:0806.1739) 
Hopkins, P. F., Hernquist, L., Cox, T. J., Dutta, S. N., \& Rothberg, B. 2008d, ApJ, 679, 156 (Paper I)

Hopkins, P. F., Hernquist, L., Cox, T. J., Keres, D., \& Wuyts, S. 2008e, ApJ, submitted (arXiv:0807.2868)

Hopkins, P. F., Hernquist, L., Cox, T. J., Robertson, B., \& Krause, E. 2007a, ApJ, 669, 45

Hopkins, P. F., Hernquist, L., Cox, T. J., Younger, J. D., \& Besla, G. 2008f, ApJ, submitted (arXiv:0806.2861)

Hopkins, P. F., Lauer, T. R., Cox, T. J., Hernquist, L., \& Kormendy, J. 2008g, ApJ, submitted (arXiv:0806.2325) (Paper III)

Hopkins, P. F., Lidz, A., Hernquist, L., Coil, A. L., Myers, A. D., Cox, T. J., \& Spergel, D. N. 2007b, ApJ, 662, 110

James, P., Bate, C., Wells, M., Wright, G., \& Doyon, R. 1999, MNRAS, 309, 585

Jorgensen, I., Franx, M., \& Kjaergaard, P. 1993, ApJ, 411, 34 1996, MNRAS, 280, 167

Joseph, R. D., \& Wright, G. S. 1985, MNRAS, 214, 87

Kannappan, S. J. 2004, ApJ, 611, L89

Katz, N., Weinberg, D. H., \& Hernquist, L. 1996, ApJS, 105, 19

Khochfar, S., \& Silk, J. 2006, ApJ, 648, L21

Kobayashi, C. 2004, MNRAS, 347, 740

Kormendy, J. 1977, ApJ, 218, 333

. 1985, ApJ, 295, 73

1987, in Nearly Normal Galaxies: From the Planck Time to the Present,

ed. S. M. Faber (New York: Springer), 163

1989, ApJ, 342, L63

1999, in ASP Conf. Ser. 182, Galaxy Dynamics-A Rutgers Symposium, ed. D. R. Merritt, M. Valluri, \& J. A. Sellwood (San Francisco: ASP), 124

Kormendy, J., Fisher, D. B., Cornell, M. E., \& Bender, R. 2008, ApJ, submitted

Kormendy, J., \& Freeman, K. C. 2004, in IAU Symp. 220, Dark Matter in Galaxies, ed. S. Ryder et al. (Dordrecht: Kluwer), 377

Kormendy, J., Gebhardt, K., Fisher, D. B., Drory, N., Macchetto, F. D., \& Sparks, W. B. 2005, AJ, 129, 2636

Kormendy, J., \& Sanders, D. B. 1992, ApJ, 390, L53

Kronawitter, A., Saglia, R. P., Gerhard, O., \& Bender, R. 2000, A\&AS, 144, 53

Kuhlen, M., Strigari, L. E., Zentner, A. R., Bullock, J. S., \& Primack, J. R. 2005, MNRAS, 357, 387

Laine, S., van der Marel, R. P., Lauer, T. R., Postman, M., O’Dea, C. P., \& Owen, F. N. 2003, AJ, 125, 478

Lake, G., \& Dressler, A. 1986, ApJ, 310, 605

Lauer, T. R. 1985, ApJS, 57, 473

Lauer, T. R., et al. 1995, AJ, 110, 2622

2005, AJ, 129, 2138

$2007 \mathrm{a}, \mathrm{ApJ}, 662,808$

2007b, ApJ, 664, 226

Leitherer, C., et al. 1999, ApJS, 123, 3

Liu, Y., Zhou, X., Ma, J., Wu, H., Yang, Y., Li, J., \& Chen, J. 2005, AJ, 129 , 2628

Mandelbaum, R., Seljak, U., Kauffmann, G., Hirata, C. M., \& Brinkmann, J. 2006, MNRAS, 368, 715

Maraston, C. 2005, MNRAS, 362, 799

McDermid, R. M., et al. 2006, MNRAS, 373, 906

McGaugh, S. S. 2005, ApJ, 632, 859

Mihos, J. C., \& Hernquist, L. 1994a, ApJ, 431, L9

1994b, ApJ, 437, 611

1994c, ApJ, 437, L47

1996, ApJ, 464, 641

Naab, T., Johansson, P. H., Ostriker, J. P., \& Efstathiou, G. 2007, ApJ, 658, 710

Navarro, J. F., Frenk, C. S., \& White, S. D. M. 1996, ApJ, 462, 563

Neto, A. F., et al. 2007, MNRAS, 381, 1450

Nipoti, C., Londrillo, P., \& Ciotti, L. 2003, MNRAS, 342, 501

Nipoti, C., Treu, T., \& Bolton, A. S. 2008, MNRAS, in press (arXiv:0806.0570)

Oñorbe, J., Domínguez-Tenreiro, R., Sáiz, A., Serna, A., \& Artal, H. 2005, ApJ, 632, L57

O’Shea, B. W., Nagamine, K., Springel, V., Hernquist, L., \& Norman, M. L. 2005, ApJS, 160, 1

Ostriker, J. P. 1980, Comments Astrophys., 8, 177

Padmanabhan, N., et al. 2004, NewA, 9, 329

Pahre, M. A., Djorgovski, S. G., \& de Carvalho, R. R. 1998, AJ, 116, 1591

Peletier, R. F., Davies, R. L., Illingworth, G. D., Davis, L. E., \& Cawson, M. 1990, AJ, 100, 1091
Persic, M., \& Salucci, P. 1988, MNRAS, 234, 131 1990, MNRAS, 245, 577

Persic, M., Salucci, P., \& Stel, F. 1996a, Astrophys. Lett. Commun., 33, 205 1996b, MNRAS, 281, 27

Postman, M., \& Lauer, T. R. 1995, ApJ, 440, 28

Prugniel, P., \& Simien, F. 1997, A\&A, 321, 111

Ravindranath, S., et al. 2004, ApJ, 604, L9

Riciputi, A., Lanzoni, B., Bonoli, S., \& Ciotti, L. 2005, A\&A, 443, 133

Rix, H.-W., de Zeeuw, P. T., Cretton, N., van der Marel, R. P., \& Carollo, C. M. 1997, ApJ, 488, 702

Robertson, B., Bullock, J. S., Cox, T. J., Di Matteo, T., Hernquist, L., Springel, V., \& Yoshida, N. 2006a, ApJ, 645, 986

Robertson, B., Cox, T. J., Hernquist, L., Franx, M., Hopkins, P. F., Martini, P., \& Springel, V. 2006b, ApJ, 641, 21

Robertson, B., Hernquist, L., Cox, T. J., Di Matteo, T., Hopkins, P. F., Martini, P., \& Springel, V. 2006c, ApJ, 641, 90

Rothberg, B., \& Joseph, R. D. 2004, AJ, 128, 2098

- 2006a, AJ, 131, 185

2006b, AJ, 132, 976

Sanders, D. B., \& Mirabel, I. F. 1996, ARA\&A, 34, 749

Sargent, A. I., Sanders, D. B., \& Phillips, T. G. 1989, ApJ, 346, L9

Sargent, A. I., Sanders, D. B., Scoville, N. Z., \& Soifer, B. T. 1987, ApJ, 312, L35

Schmidt, R. W., \& Allen, S. W. 2007, MNRAS, 379, 209

Scoville, N. Z., Sanders, D. B., Sargent, A. I., Soifer, B. T., Scott, S. L., \& Lo, K. Y. 1986, ApJ, 311, L47

Shankar, F., Lapi, A., Salucci, P., De Zotti, G., \& Danese, L. 2006, ApJ, 643, 14

Shapley, A. E., Coil, A. L., Ma, C.-P., \& Bundy, K. 2005, ApJ, 635, 1006

Shen, S., Mo, H. J., White, S. D. M., Blanton, M. R., Kauffmann, G., Voges, W., Brinkmann, J., \& Csabai, I. 2003, MNRAS, 343, 978

Shier, L. M., \& Fischer, J. 1998, ApJ, 497, 163

Simien, F., \& Prugniel, P. 1997, A\&AS, 122, 521 2002, A\&A, 384, 371

Soifer, B. T., et al. 1984a, ApJ, 278, L71 1984b, ApJ, 283, L1

Springel, V. 2005, MNRAS, 364, 1105

Springel, V., Di Matteo, T., \& Hernquist, L. 2005, MNRAS, 361, 776

Springel, V., \& Hernquist, L. 2002, MNRAS, 333, 649 2003, MNRAS, 339, 289

$$
\text { 2005, ApJ, 622, L9 }
$$

Thomas, D., Maraston, C., Bender, R., \& Mendes de Oliveira, C. 2005, ApJ, 621,673

Toomre, A. 1977, in Evolution of Galaxies and Stellar Populations, ed. B. M. Tinsley \& R. B. Larson (New Haven: Yale Univ. Obs.), 401

Toomre, A., \& Toomre, J. 1972, ApJ, 178, 623

Trager, S. C., Faber, S. M., Worthey, G., \& González, J. J. 2000, AJ, 119, 1645

Treu, T., et al. 2005, ApJ, 633, 174

Trujillo, I., Burkert, A., \& Bell, E. F. 2004, ApJ, 600, L39

Trujillo, I., et al. 2006, ApJ, 650, 18

Vale, A., \& Ostriker, J. P. 2006, MNRAS, 371, 1173

van den Bosch, F. C., et al. 2007, MNRAS, 376, 841

van der Marel, R. P. 1991, MNRAS, 253, 710

van der Wel, A., Franx, M., van Dokkum, P. G., Rix, H.-W., Illingworth, G. D., \& Rosati, P. 2005, ApJ, 631, 145

van Dokkum, P. G., \& van der Marel, R. P. 2007, ApJ, 655, 30

Vitvitska, M., Klypin, A. A., Kravtsov, A. V., Wechsler, R. H., Primack, J. R., \& Bullock, J. S. 2002, ApJ, 581, 799

von der Linden, A., Best, P. N., Kauffmann, G., \& White, S. D. M. 2007, MNRAS, 379, 867

Wang, L., Li, C., Kauffmann, G., \& de Lucia, G. 2006, MNRAS, 371, 537

Wechsler, R. H., Bullock, J. S., Primack, J. R., Kravtsov, A. V., \& Dekel, A. 2002, ApJ, 568, 52

Weinmann, S. M., van den Bosch, F. C., Yang, X., \& Mo, H. J. 2006, MNRAS, 366,2

Yang, X., Mo, H. J., Jing, Y. P., \& van den Bosch, F. C. 2005, MNRAS, 358, 217 Younger, J. D., Hopkins, P. F., Cox, T. J., \& Hernquist, L. 2008, ApJ, 686, 815 Zentner, A. R., Berlind, A. A., Bullock, J. S., Kravtsov, A. V., \& Wechsler, R. H. 2005, ApJ, 624, 505

Zheng, Z., et al. 2005, ApJ, 633, 791

Zirm, A. W., et al. 2007, ApJ, 656, 66 